\title{
Coarse Geometry for Noncommutative Spaces
}

\author{
Dissertation \\ zur Erlangung des mathematisch-naturwissenschaftlichen \\ Doktorgrades \\ „Doctor rerum naturalium“ (Dr.rer.nat.) \\ der Georg-August-Universität Göttingen \\ im Promotionsprogramm der PhD School of Mathematical Science (SMS) \\ der Georg-August University School of Science (GAUSS) \\ vorgelegt von \\ Tathagata Banerjee \\ aus West Bengal, Indien
}


Betreungsausschuss:

Betreuer: Prof. Dr. Ralf Meyer

Mathematisches Institut, Georg-August-Universität Göttingen

Betreuer: Prof. Dr. Thomas Schick

Mathematisches Institut, Georg-August-Universität Göttingen

Mitglieder der Prüfungskommission:

Referent: Prof. Dr. Ralf Meyer

Mathematisches Institut, Georg-August-Universität Göttingen Koreferent: Prof. Dr. Thomas Schick

Mathematisches Institut, Georg-August-Universität Göttingen

Prof. Dr. Gert Lube

Institut für Numerische und Angewandte Mathematik,

Georg-August-Universität Göttingen

Prof. Dr. Dorothea Bahns

Mathematisches Institut, Georg-August-Universität Göttingen

Prof. Dr. Karl-Henning Rehren

Institut für Theoretische Physik, Georg-August-Universität Göttingen

Prof. Dr. Ingo Witt

Mathematisches Institut, Georg-August-Universität Göttingen 
To my parents, grandparents and late Bomma... 



\section{Acknowledgments}

My heartfelt thanks to everybody who has contributed to my growth towards this doctoral degree. At the very beginning I would like to express my deepest gratitude to Prof. Thomas Schick. Several interactions with him and his wonderful teaching in mathematics have inspired me to pursue my endeavours in mathematics. I am also highly indebted to my supervisor Prof. Ralf Meyer for his extreme patience and guidance throughout the period of my work on this project. Apart from this, a major thanks to Dr. Jianchao Wu for several discussions, giving key insights on my thesis project and his immense hospitality during my visit to Münster.

I would like to thank the Research Training Group 1493 for awarding me the doctoral stipend during the first three years of my work along with generous grants for visits to conferences. Also I am much obliged to my supervisors Prof. Ralf Meyer and Prof. Thomas Schick for granting me extensions to complete my thesis. Thanks to Prof. Chenchang Zhu for bearing my travel and registration fees to conferences from her grant, during the last six months of my work.

Apart from this a major thanks goes to my friend and colleague Dr. Suliman Albandik for always being there to help me when I needed. Also a big thanks to the rest of my colleagues and professors in Göttingen with special mention to Rudolf Zeidler for certain fruitful discussions on my thesis project. A big thanks to my friends and family whose caring and support has made this possible. Finally, my sincere thanks to Prof. Mahan Mj under whose care and guidance I had the blessing to start my journey in mathematics. 


\begin{abstract}
We develop an analogoue of coarse geometry for noncommutative spaces in terms of unitizations of the given $\mathrm{C}^{*}$-algebra. Examples for our theory come from Rieffel deformation of compactifications under strongly continuous actions of $\mathbb{R}^{d}$. A special case of this is the coarse structure on the Moyal plane, seen as a Rieffel deformation of the classical plane. The motivating question for this project has been to investigate a possible coarse equivalence between the classical plane and the Moyal plane, which seems plausible in physics. We define a noncommutative analogue of coarse maps. Our definition ensures that the classical and the Moyal plane with their standard coarse structures are coarsely equivalent. A more general result holds for Rieffel deformations of arbitrary actions of $\mathbb{R}^{d}$ by translations.
\end{abstract}




\section{Contents}

\begin{tabular}{lll}
\hline 0 & Introduction & 1
\end{tabular}

1 Noncommutative coarse structures 5

1.1 Coarse structures . . . . . . . . . . . . . . . . . . 5

1.1.1 The abstract notion of a coarse structure . . . . . . . 5

1.1 .2 Topological coarse structures $\ldots \ldots \ldots \ldots \ldots$

1.2 Noncommutative coarse structures $\ldots \ldots \ldots \ldots \ldots$

1.2 .1 Unitization of $\mathrm{C}^{*}$-algebras $\ldots \ldots \ldots \ldots \ldots$

1.2 .2 The Gelfand-Naimark correspondence . . . . . . . . . 11

1.2 .3 Compactifications and unitizations . . . . . . . . . 12

1.3 Boundaries of compactifications . . . . . . . . . . . . . 14

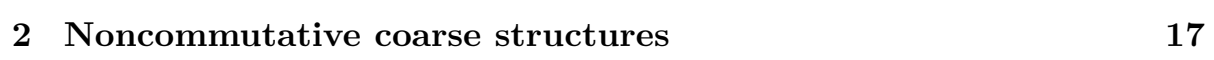

2.1 Rieffel deformation . . . . . . . . . . . . . . . . . . . . . . . 17

$2.1 .1 \quad$ Extending Rieffel deformation, crossed products $\ldots . .20$

$2.2 \quad$ Rieffel deformation via crossed products $\ldots \ldots \ldots \ldots . \ldots 23$

$2.2 .1 \quad G$-product . . . . . . . . . . . . . . . . 23

2.2 .2 Landstad algebra of a $G$-product . . . . . . . . . . 24

$2.2 .3 \quad$ Deformation using a 2-cocycle on the dual group . . . . . 25

2.2 .4 The special case of a trivial action . . . . . . . . 26

$2.3 \quad$ Examples of noncommutative coarse structures . . . . . . . . 29

$2.3 .1 \quad$ Deformation procedure preserves exactness $\ldots \ldots \ldots .29$

$2.3 .2 \quad$ Noncommutative coarse structure on the Moyal Plane . . 32

\begin{tabular}{|lll}
3 & Noncommutative coarse maps & 37
\end{tabular}

$3.1 \quad$ Noncommutative coarse maps . . . . . . . . . . . . . . . 37 
3.1.1 Hilbert $\mathrm{C}^{*}$-modules and completely positive maps . . . . 38

3.2 Coarse maps . . . . . . . . . . . . . . . . . . . . 41

3.2.1 Continuous coarse maps for topological coarse structures. 43

$3.3 \quad$ Discontinuous coarse maps . . . . . . . . . . . . . . . . . . . . . . 44

$3.3 .1 \quad$ Functoriality of the Higson corona . . . . . . . . . . . . . 48

3.3 .2 Discontinuous coarse maps for topological coarse structures 50

$3.3 .3 \quad$ More coarse maps . . . . . . . . . . . . . . . 54

3.4 Noncommutative coarse equivalence . . . . . . . . . . . . . 55

$3.4 .1 \quad$ Noncommutative coarse maps for Rieffel deformation. . . 58

$3.4 .2 \quad$ An explicit noncommutative coarse map . . . . . . . . . . 58

$3.4 .3 \quad$ Noncommutative coarse equivalence between the classical plane and the Moyal plane . . . . . . . . . . . . . . 61 


\section{Chapter 0}

\section{Introduction}

Coarse geometry studies large-scale properties of topological spaces. What could coarse geometry mean for noncommutative spaces? Boundaries play a major role in many coarse geometric studies. Moreover, there is a particular coarse structure determined by a boundary. This treatment of what we call a noncommutative coarse structure on a non-unital $\mathrm{C}^{*}$-algebra by starting with an appropriate boundary has been the major theme of my thesis work.

One of the major motivations of our work on noncommutative coarse structures has been to understand the coarse structure underlying the quantum plane. It is quite well-known from physics that the main difference between quantum and classical phenomena lies in the small scale of objects involved. On large scales, their differences should disappear. This led us to seek a notion of coarse structure for the Moyal plane which should then be shown to be equivalent to the classical plane.

Following Roe [9], any coarse structure on a locally compact Hausdorff space $X$ is defined by a collection of subsets of $X \times X$, satisfying certain axioms. These subsets $E \subset X \times X$ are called controlled. They determine which pairs of points are to be considered uniformly close.

Let us briefly look at the category of coarse structures on locally compact Hausdorff spaces with coarse maps as morphisms. Within it, consider the following two parts, that of proper coarse structures and the other of topological coarse structures coming from a compactification. These two are related in the sense that both map to the category of topological spaces and continuous maps in a similar manner. Given a proper coarse structure, one can construct a compactification called the Higson compactification such that the boundary, the Higson corona, defines a functor to the category of compact topological spaces and continuous maps. Whereas for objects corresponding to topological coarse structures, the boundary of the compactification is a functor to the category of compact topological spaces.

Given an arbitrary noncommutative $\mathrm{C}^{*}$-algebra $A$, the pure state space $\mathcal{P}(A)$, corresponding to the underlying topological space in the commutative case, can be topologically very bad. Thus in the noncommutative setting, since the 
underlying topology is not good enough, we do not expect to define a coarse structure in terms of controlled sets. Rather, as motivated in the paragraph above, we shall use the boundary coming from a unitization of $A$ to determine its coarse structure. For certain noncommutative $\mathrm{C}^{*}$-algebras $A$ and their unitizations $\bar{A}$, the quotient algebra $\bar{A} / A$ is isomorphic to a commutative boundary like $C(\bar{X}) / C_{0}(X)$. Here $\bar{X}$ is some compactification of a locally compact Hausdorff space $X$. This allows us to compare the noncommutative coarse space given by $A \triangleleft \bar{A}$ to the classical coarse space $X \subseteq \bar{X}$.

So in our work, a noncommutative coarse structure on a non-unital $\mathrm{C}^{*}$-algebra $A$ is defined as a unitization $\bar{A}$ of the $\mathrm{C}^{*}$-algebra $A$. Defining coarse structures in terms of compactifications raises questions regarding well-definedness of such an object as we go back from the topological category to the coarse category. In the case of metric coarse structures on $(X, d)$, by a theorem of Roe $[9]$, the topological coarse structure coming from the Higson compactification of a proper metric $d$ coincides with the original metric coarse structure. With this, we use the Higson compactification from the metric coarse structure on the Euclidean space $\mathbb{R}^{2 n}$ to define a coarse structure on the classical plane uniquely.

The Moyal plane is a Rieffel deformation of the classical plane $\mathbb{R}^{2 n}$ under the translation action of the vector group $\mathbb{R}^{2 n}$. Using that exactness is preserved under Rieffel deformation, we define the coarse structure on the Moyal plane, as a Rieffel deformation of the Higson compactification of $\mathbb{R}^{2 n}$. Since the translation action is trivial on the boundary of the Higson compactification, its Rieffel deformation, which is a boundary to the Moyal plane, is isomorphic to the original commutative boundary. We therefore expect that the coarse structure on the Moyal plane defined by this process is equivalent to that on the classical plane.

In this respect, it should be mentioned that to define the coarse structure, we use functoriality of the deformation process in Kasprzak's equivalent approach to Rieffel deformation [4]. For a trivial action, we show that the deformed algebra is in fact the same as the original algebra. We show that for topological coarse structures, with the metric coarse structure on the range space, a coarse map inducing a homeomorphism between the boundaries gives a coarse equivalence.

In the classical case, a map $\phi: X \rightarrow Y$ is coarse if it is proper and bornologous. The properness condition means that points on the boundary are mapped only to the boundary, and the bornologous condition for topological coarse structures means that the map induced between the boundaries is continuous. Let $0 \rightarrow A \rightarrow \bar{A} \rightarrow \bar{A} / A \rightarrow 0$ and $0 \rightarrow B \rightarrow \bar{B} \rightarrow \bar{B} / B \rightarrow 0$ be certain extensions by unitizations $\bar{A}, \bar{B}$ that define noncommutative coarse structures on the nonunital $\mathrm{C}^{*}$-algebras $A, B$, respectively. We call a strictly continuous, contractive, completely positive map $\phi: A \rightarrow B$ a noncommutative coarse map if it extends to a unital completely positive map between the multiplier algebras with the following properties. Since the coarse structures are determined specifically by the unitizations $\bar{A}, \bar{B}$, respectively, we require $\bar{\phi}$ to restrict to a strictly continuous map between these given unitizations. Furthermore this restriction map needs to induce a $*$-homomorphism between the boundary quotient algebras $[\bar{\phi}]: \bar{A} / A \rightarrow \bar{B} / B$. We say the following commuting diagram below defines a 
noncommutative coarse map:

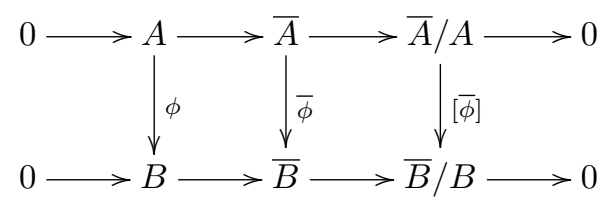

If $\phi: A \rightarrow B$ is strictly continuous, completely positive, contractive then the induced map between the dual spaces maps pure states to states. Now states on a $\mathrm{C}^{*}$-algebra are equivalent to probability measures in the commutative case. Thus, in effect we have a measure-valued map that converges to a pointvalued map at the boundary. As shall be evident later, the main challenge is to incorporate discontinuous coarse maps in the algebraic picture between the $\mathrm{C}^{*}$-algebras of continuous functions. Thus we consider the bigger class of completely positive maps as noncommutative coarse maps. *-homomorphisms between the algebras correspond to continuous coarse maps.

Starting with a discontinuous coarse map, Roe develops an equivalent measurevalued map in 8 that gives a continuous map between the Higson coronas. The conditions on the measure-valued map and that it restricts to a $*$-homomorphism at the boundary say that given a net of points in the domain converging to the boundary, the image net of measures also converges to a boundary point. But the support of the measures in the image net can be the whole of the space, and the convergence condition only says that a certain 'major volume' of the measures in the image net should converge to the boundary point. But it is still not clear how to quantify this major volume, which starting with a measure-valued map could help us to construct a close point-valued coarse map. Thus the opposite direction to show that such a measure-valued map between topological coarse structures always gives a point-valued coarse map, up to an equivalence condition of closeness, is yet to be understood. Until then, our definition of a noncommutative coarse map may contain more coarse maps in the commutative case.

We next consider the equivalence relation of closeness between two noncommutative coarse maps. We call two coarse completely positive maps $\phi_{1}, \phi_{2}: A \rightarrow B$ close if they induce the same $*$-homomorphism between the boundary quotients, that is, $\left[\overline{\phi_{1}}\right]=\left[\overline{\phi_{2}}\right]$. In classical coarse geometry two coarse maps $f, g: X \rightarrow Y$ are close if the set $\{(f(x), g(x)): \forall x \in X\}$ is a controlled set in $Y$. For topological coarse structures coming from compactifications, this condition is equivalent to that they extend to the same map between the boundaries of the compactification.

With the equivalence relation of closeness between two maps, a coarse map $f: X \rightarrow Y$ is a coarse equivalence if there exists another coarse map $g: Y \rightarrow X$ such that $f \circ g$ is close to $\operatorname{id}_{Y}$ and $g \circ f$ is close to $\mathrm{id}_{X}$. It is trivial to show that a coarse equivalence between proper coarse structures gives a homeomorphism between the Higson coronas, as would be expected from the functoriality of the Higson corona. For topological coarse structures, a coarse equivalence implies that they have homeomorphic boundaries. In the other direction, we consider the special case of a coarse map $f: X \rightarrow Y$ to a metric coarse structure on $Y$, like in the example of the classical plane. Then we show that if $f$ extends to a 
homeomorphism between the boundaries, it is actually a coarse equivalence.

We call a coarse completely positive map $\phi: A \rightarrow B$ a noncommutative coarse equivalence if there exists a coarse completely positive map $\Psi: B \rightarrow A$ in the opposite direction such that they both restrict to $*$-isomorphisms between the boundary quotient algebras that are inverse to each other. With this we construct a noncommutative coarse equivalence between the classical plane and the Moyal plane, with noncommutative coarse structures as defined before.

For this we use the completely positive quantization maps between Rieffel deformations as developed in [2] by Kascheck, Neumaier and Waldmann. There is such a map on the Higson compactification of the bounded coarse structure on $\mathbb{R}^{2 n}$ coming from the Euclidean metric. This restricts to a completely positive map on the ideal $C_{0}\left(\mathbb{R}^{2 n}\right)$ mapping to the Moyal plane. Using triviality of the translation action of $\mathbb{R}^{2 n}$ on the Higson corona, we then show that it induces the identity $*$-isomorphism between the boundary quotient algebras, as required by our theory.

In the first chapter, we introduce noncommutative coarse structures on $\mathrm{C}^{*}$-algebras. Starting with the classical treatment of coarse geometry on topological spaces, a brief discourse is then given on the preliminary $\mathrm{C}^{*}$-algebra theory we need to understand the definition of a noncommutative coarse structure on a $\mathrm{C}^{*}$-algebra. This includes Gelfand-Naimark theory on concrete representations of abstract $\mathrm{C}^{*}$-algebras and the correspondence between compactifications and unitizations. The minimal and maximal unitizations of a $\mathrm{C}^{*}$-algebra is treated in detail. In the final section we discuss the importance of the boundary quotient algebra of a given unitization in determining the noncommutative coarse structure.

In the second chapter, we discuss Rieffel deformation and the related machinery we use, and then apply it to define a noncommutative coarse structure on the Moyal plane. Using Kasprzak's theory of Rieffel deformation, we prove here that for a trivial action of the group the deformed algebra is the same as the undeformed $\mathrm{C}^{*}$-algebra. In the end, we apply Rieffel deformation to the metric coarse structure of the classical plane to define a noncommutative coarse structure on the Moyal plane. Using triviality of the translation action on the Higson corona, we expect this noncommutative coarse structure of the Moyal plane to be equivalent to the metric coarse structure on $\mathbb{R}^{2 n}$ by our definition of a noncommutative coarse structure.

In the third chapter, we define noncommutative coarse maps and compare them with the classical coarse maps. We also discuss in detail the problems that still need to be solved. Finally, we study the completely positive quantization map between Rieffel deformations developed in $[2$. With this we establish the coarse equivalence of the classical plane and the Moyal plane. 


\section{Chapter 1}

\section{Noncommutative coarse structures}

Coarse geometry or large-scale geometry studies the macroscopic features of a given topology. Coarse structures are closely related to compactifications. There are natural constructions going back and forth between coarse structures and compactifications, which are often inverse to each other (but not always). The noncommutative analogue of a coarse structure is a unitization of a $\mathrm{C}^{*}$-algebra. We use these to define coarse structures on $\mathrm{C}^{*}$-algebras.

\subsection{Coarse structures}

Let us start with a brief introduction to what is coarse geometry in line with Prof. John Roe's axiomatic definition of coarse structures as in [9]. Coarse geometry concerns itself with loosening the concept of distance in spaces. Several metrics may give the same large-scale geometry on the space. The idea is to formalize when two metrics are to be considered equivalent from this aspect. Here we are mostly concerned with understanding what coarse geometry means for a topological space to help us extend the notion to noncommutative spaces.

\subsubsection{The abstract notion of a coarse structure}

Let $X$ be a set. We will use the following notation for subsets of $X \times X$.

1. If $E \subseteq X \times X$, then $E^{-1}$ denotes the set $\left\{\left(x, x^{\prime}\right):\left(x^{\prime}, x\right) \in E\right\}$, which is called the inverse of $E$;

2. If $E^{\prime}, E^{\prime \prime} \subseteq X \times X$, then

$$
E^{\prime} \circ E^{\prime \prime}:=\left\{\left(x^{\prime}, x^{\prime \prime}\right): \exists x \in X,\left(x^{\prime}, x\right) \in E^{\prime},\left(x, x^{\prime \prime}\right) \in E^{\prime \prime}\right.
$$

is called the product of $E^{\prime}$ and $E^{\prime \prime}$. 
If $E \subseteq X \times X$ and $K \subseteq X$ we define

$$
E[K]:=\left\{x^{\prime}: \exists x \in K,\left(x^{\prime}, x\right) \in E\right\} .
$$

Definition 1.1. A coarse structure on a set $X$ is a collection $\mathcal{E}$ of subsets of $X \times X$, called controlled sets or entourages, which contains the diagonal and is closed under the formation of subsets, inverses, products, and (finite) unions. A set equipped with a coarse structure is called a coarse space.

The most important example is the natural coarse structure on a metric space.

Example 1.2. Let $(X, d)$ be a metric space and let $\mathcal{E}$ be the collection of all those subsets $E \subseteq X \times X$ for which $\sup \left\{d\left(x^{\prime}, x\right):\left(x^{\prime}, x\right) \in E\right\}$ is finite. Then $\mathcal{E}$ is a coarse structure. It is called the bounded coarse structure of the metric or the bounded metric coarse structure on $(X, d)$. It is equal to the coarse structure generated ${ }^{1}$ by the sets $E_{r}:=\left\{\left(x, x^{\prime}\right) \in X \times X: d\left(x, x^{\prime}\right) \leq r\right\}$ for all $r>0$.

The axiomatic definition of a coarse structure is useful and defines the broad set-up one needs to look at coarse geometry. Within this framework one can consider several different coarse structures on $X$. We are interested in coarse structures on a non-unital $\mathrm{C}^{*}$-algebra, seen as an extension of coarse structures on topological spaces. Therefore, we need a special subclass of coarse structures, namely, those that are compatible with a topology. These have wide applications to problems in topology and hence are also important from this point of view.

They are known as proper coarse structures and put an extra restriction on the underlying topology of $X$. Apart from $X$ being locally compact and Hausdorff, something that is essential for generalizing to $\mathrm{C}^{*}$-algebras, $X$ should also be paracompact.

Definition 1.3. A coarse structure on a paracompact Hausdorff space $X$ is proper if

(i) there is a controlled neighbourhood of the diagonal, and

(ii) every bounded subset of $X$ is relatively compact, where $B \subset X$ is a bounded subset of $X$ if $B \times\{p\}$ is controlled for some $\{p\} \in X$.

Example 1.4. Let $(X, d)$ be a metric space. Its metric coarse structure is proper if and only if it is proper as a metric space, that is, closed bounded subsets with respect to the metric are compact.

Given a proper coarse structure, one can define a particular compactification called the Higson compactification, a helpful construction to pass from the category of proper coarse structures to that of compact topological spaces.

Definition 1.5. Let $X$ be a proper coarse space (a paracompact space with a proper coarse structure) and let $f: X \rightarrow \mathbb{C}$ be a bounded continuous function. We denote by $\mathbf{d} f$ the function

$$
\mathbf{d} f(x, y)=f(y)-f(x): X \times X \rightarrow \mathbb{C} .
$$

\footnotetext{
${ }^{1}$ This is the smallest collection of subsets of $X \times X$ that contains all the generating sets and also satisfies the axioms of a coarse structure.
} 
We call $f$ a Higson function if, for the closure of each controlled set $\bar{E}$, the restriction of $\mathbf{d} f$ to $\bar{E}$ vanishes at the boundary; that is $\mathbf{d} f \in C_{0}(\bar{E})$.

Proposition 1.6. 9, Proposition 2.36] The Higson functions on a proper coarse space $X$ form a unital $C^{*}$-subalgebra of $C_{b}(X)$ containing the essential ideal $C_{0}(X)$. Let $C_{h}(X)$ be the set of Higson functions on $X$.

Proof. The only non-trivial part is to see that the product of two Higson functions is a Higson function. This follows from $\mathbf{d}(f g)(x, y)=\mathbf{d} f(x, y) g(x)+$ $f(y) \mathbf{d} g(x, y)$. For any $f \in C_{0}(X)$, the Higson condition is trivial, so $C_{0}(X) \subset$ $C_{h}(X)$.

Definition 1.7. By the Gelfand-Naimark Theorem ${ }^{2} C_{h}(X)$ corresponds to a compactification $h X$ of $X$ called the Higson compactification.

\subsubsection{Topological coarse structures}

These are one of the major objects we use for our project on noncommutative coarse structures. They are the coarse structures on a locally compact Hausdorff space that are defined in terms of a compactification. For now, $X$ denotes a paracompact and locally compact Hausdorff space.

Definition 1.8. A compactification of the locally compact Hausdorff space $X$ is a compact Hausdorff space $\bar{X}$ that contains $X$ as a dense open subset. The compact subspace $\partial X=\bar{X} \backslash X$ is called the boundary of the compactification.

As we shall see in more detail later, a compactification $\bar{X}$ of $X$ is completely described by the algebra $C(\bar{X})$ of continuous (complex-valued) functions on it. Each such function is uniquely determined by its restriction to $X$, and thus $C(\bar{X})$ may be identified with a $\mathrm{C}^{*}$-subalgebra of $C_{b}(X)$, the algebra of all bounded continuous functions on $X$. Two extreme examples are:

(i) the one-point compactification of $X$, corresponding to the $\mathrm{C}^{*}$-algebra $C_{0}(X)^{\dagger}$ of functions that tend to a constant value at infinity;

(ii) the Stone- $\check{C} e c h$ compactification, corresponding to the $\mathrm{C}^{*}$-algebra of all bounded continuous functions on $X$.

The construction in the theorem below defines a coarse structure on $X$. We write down the proof from [9] to give an idea of the workings behind the main object of our study.

Theorem 1.9. 9, Theorem 2.27] Let $X$ be a paracompact and locally compact Hausdorff space with a compactification $\bar{X}$. Let $E \subseteq X \times X$. The following conditions are equivalent.

(i) The closure $\bar{E}$ of $E$ in $\bar{X} \times \bar{X}$ meets the complement of $X \times X$ only in the diagonal $\Delta_{\partial X}=\{(\omega, \omega): \omega \in \partial X\}$.

\footnotetext{
${ }^{2}$ We expect the reader to be aware of it though for our current work we shall also treat this theorem in detail later.
} 
(ii) $E$ is proper ${ }^{3}$, and for every net $\left(x_{\lambda}, y_{\lambda}\right)$ in $E$, if $\left\{x_{\lambda}\right\}$ converges to a point $\omega \in \partial X$, then $\left\{y_{\lambda}\right\}$ also converges to $\omega$.

(iii) $E$ is proper, and for every point $\omega \in \partial X$ and every neighbourhood $V$ of $\omega$ in $\bar{X}$, there is a neighbourhood $U \subseteq V$ of $\omega$ in $\bar{X}$ with $E \cap(U \times(X \backslash V))=\emptyset$.

Moreover, the sets E satisfying these equivalent conditions form the controlled sets for a connected $d^{\sqrt{4}}$ coarse structure on $X$.

Proof. It is immediate that (i) implies (ii).

To show that (ii) implies (iii), suppose that (iii) is false. Then there is $\omega \in \partial X$ and a neighbourhood $V$ of $\omega$, such that for every neighbourhood $U$ of $\omega$ there is a point $\left(x_{U}, y_{U}\right) \in E \cap(U \times(X \backslash V))$. The assignment $U \mapsto\left(x_{U}, y_{U}\right)$ is a net and $\left\{x_{U}\right\}$ converges to $\omega$. Since $\left\{y_{U}\right\}$ belongs to the closed set $X \backslash V$, which does not contain $\omega$, it is impossible for $\left\{y_{U}\right\}$ to converge to $\omega$, and this contradicts (ii).

To show that (iii) implies (i), let $\left(\omega, \omega^{\prime}\right)$ be a point of $\bar{E} \backslash X \times X$. The properness of $E$ implies that $\left(\omega, \omega^{\prime}\right) \in \partial X \times \partial X$. Suppose for a contradiction that $\omega \neq \omega^{\prime}$. Fix disjoint neighbourhoods $V, V^{\prime}$ of $\omega, \omega^{\prime}$; then for any neighbourhood $U \subseteq V$ of $\omega$ we have $\left(\omega, \omega^{\prime}\right) \in U \times V^{\prime}$, so $E$ must meet $U \times V^{\prime} \subseteq U \times(X \backslash V)$. This contradicts (iii).

To show that the class $\mathcal{E}$ of sets $E$ characterized by these conditions forms a coarse structure, we use condition (i) to see that $\mathcal{E}$ is closed under the formation of inverses, subsets, and finite unions. To see that $\mathcal{E}$ is closed under the formation of products, it is easiest to use condition (ii). Suppose that $\left\{\left(x_{\lambda}, z_{\lambda}\right)\right\}$ is a net in $E=E^{\prime} \circ E^{\prime \prime}$, where $E^{\prime}, E^{\prime \prime} \in \mathcal{E}$. For each $\lambda$ there is $y_{\lambda}$ such that $\left(x_{\lambda}, y_{\lambda}\right) \in E^{\prime}$ and $\left(y_{\lambda}, z_{\lambda}\right) \in E^{\prime \prime}$. Suppose that $\left\{x_{\lambda}\right\}$ converges to $\omega \in \partial X$. Then $\left\{y_{\lambda}\right\}$ converges to $\omega$ because $E^{\prime} \in \mathcal{E}$, and then $\left\{z_{\lambda}\right\}$ converges to $\omega$ because $E^{\prime \prime} \in \mathcal{E}$. It follows that $E \in \mathcal{E}$.

Any compact subset of $X \times X$ belongs to $\mathcal{E}$. In particular, the compact set $\left\{x, x^{\prime}\right\}$ belongs to $\mathcal{E}$ for all $x, x^{\prime}$ in $X$, so the coarse structure $\mathcal{E}$ is connected.

Definition 1.10. The coarse structure described in Theorem 1.9 is called the topological coarse structure associated to the given compactification.

We shall repeatedly need the topological coarse structure to be proper. Therefore, we give the proof for the corrected statement in [1].

Lemma 1.11. The topological coarse structure associated to a second countable compactification is proper.

Proof. Since every controlled set is proper in $X \times X$, every bounded subset of $X$ has compact closure. We must produce a controlled neighbourhood of the diagonal. Using Urysohn's Lemma, choose continuous functions $f, g: \bar{X} \times \bar{X} \rightarrow$ $\mathbb{R}^{+}$such that $f$ vanishes only on the diagonal, and $g$ vanishes only at infinity (that is, on $\bar{X} \times \partial X \cup \partial X \times \bar{X}$ ). Let

\footnotetext{
${ }^{3}$ A subset $E \subset X \times X$ is proper if $E[K]$ and $E^{-1}[K]$ are relatively compact whenever $K$ is relatively compact. This is not something very important for our work and is only used here to show that the coarse structure defined is connected.

${ }^{4} \mathrm{~A}$ coarse structure is connected if every point in $X \times X$ belongs to some controlled set.
} 


$$
E=\{(x, y) \in X \times X: f(x, y)<g(x, y)\} .
$$

Then $E$ is open and contains the diagonal of $X \times X$. Moreover, if $\left(x_{\lambda}, y_{\lambda}\right)$ is a net in $E$ and $x_{\lambda} \rightarrow \omega \in \partial X$, then $g\left(x_{\lambda}, y_{\lambda}\right) \rightarrow 0$ and thus $f\left(x_{\lambda}, y_{\lambda}\right) \rightarrow 0$ also. It follows that $y_{\lambda}$ converges to $\omega$; so $E \in \mathcal{E}$.

Later in Chapter 3 we show in Lemma 3.23 that the second countability restriction on the compactification $\bar{X}$ can be weakened to a certain extent. We consider $\sigma$-compact spaces with compactifications $\bar{X}$ such that the boundary $\partial \bar{X}$ is second countable. In this more general context we show in Chapter 3 that all the major results in [9, Chapter 2] follow through.

The following example compares a well-known example of a bounded metric coarse structure with a topological coarse structure coming from a well-known compactification.

Example 1.12. The bounded coarse structure associated to the Euclidean metric on $\mathbb{R}^{n}$ is strictly finer ${ }^{5}$ than the topological coarse structure associated to its compactification by a sphere $S^{n-1}$ of points at infinity.

Before ending this section on coarse geometry, let us state one of the main theorems in [9] we use in our work. We use this theorem to define a noncommutative coarse structure on the Moyal plane equivalent to the bounded metric coarse structure on the classical plane $\mathbb{R}^{2 n}$.

Theorem 1.13. 9, Proposition 2.47] Let $(X, d)$ be a proper metric space. Then the bounded metric coarse structure on $X$ is the topological coarse structure associated to its Higson compactification.

In the opposite direction, we have the following important theorem.

Theorem 1.14. 9, Proposition 2.48] Suppose that $X$ is a locally compact Hausdorff space, and that it is given the topological coarse structure associated to a second countable compactification $\bar{X}$. Then the Higson compactification of $X$ is the originally given compactification $\bar{X}$.

Having given a basic idea to the required amount of coarse geometry concepts we need for this project, we next introduce noncommutative coarse structures. This is our main object of study.

\subsection{Noncommutative coarse structures}

The plan for this project has been to imitate the framework of coarse geometry in the realm of commutative $\mathrm{C}^{*}$-algebras and then extend to noncommutative $\mathrm{C}^{*}$-algebras, as much as possible. As we shall see in detail below, the underlying topological space of a noncommutative $\mathrm{C}^{*}$-algebra is not a very well-defined object. There are several candidates that can be considered, and even then most

\footnotetext{
${ }^{5}$ If $\mathcal{E} \subset \mathcal{F}$, so that every $\mathcal{E}$-controlled set is also $\mathcal{F}$-controlled, then we call $\mathcal{E}$ finer than $\mathcal{F}$.
} 
of such topologies are without much structure. Thus we do not expect to imitate a set-theoretic definition of coarse structure for noncommutative $\mathrm{C}^{*}$-algebras.

This makes us look at topological coarse structures on a topological space $X$ coming from a compactification $\bar{X}$. In the more general category of $\mathrm{C}^{*}$-algebras, unitizations of non-unital $\mathrm{C}^{*}$-algebras generalize the notion of compactifications of locally compact Hausdorff spaces. With this, we then define a noncommutative coarse structure by a unitization of the given non-unital $\mathrm{C}^{*}$-algebra.

We next take a short discourse through the important notions of $\mathrm{C}^{*}$-algebra theory which we need to understand better this correspondence between coarse geometry and noncommutative coarse structures for $\mathrm{C}^{*}$-algebras via unitizations. We shall only assume the reader knows the definition of an abstract $\mathrm{C}^{*}$-algebra from the viewpoint of functional analysis.

\subsubsection{Unitization of $\mathrm{C}^{*}$-algebras}

Let us start with the notion of a unitization of a $\mathrm{C}^{*}$-algebra. Later, we shall establish its connection with compactifications in the commutative case.

Definition 1.15. An ideal $I$ in a $\mathrm{C}^{*}$-algebra $A$ is essential if each non-zero closed ideal of $A$ has a non-zero intersection with $I$. Equivalently, the annihilator $I^{\perp}:=\{a \in A: a I=0\}$ is zero.

In the commutative case, consider an open subset $Y \subset X$ such that $Y$ is not dense in $X$. Then $\bar{Y} \neq X$, where $\bar{Y}$ is the closure of $Y$ in $X$. Since $X$ is compact Hausdorff, it is normal. Thus by Urysohn's Lemma for any $x \in X$ and $x \notin \bar{Y}$, there exists a continuous function $f_{x} \in C(X)$ such that $f_{x}(\bar{Y})=0$ and $f_{x}(x)=1$. Thus we have a non-zero element $f_{x} \in C_{0}(Y)^{\perp} \subset C(X)$. So $C_{0}(Y)$ is not an essential ideal.

Definition 1.16. Given a locally compact space $X$, a compactification of $X$ is a compact space $\bar{X}$ such that $C(\bar{X})$ contains $C_{0}(X)$ as an essential ideal. $\bar{X}$ being Hausdorff shall imply $X$ is Hausdorff.

This definition generalizes directly to noncommutative $\mathrm{C}^{*}$-algebras.

Definition 1.17. Given a non-unital $\mathrm{C}^{*}$-algebra $A$, a unitization of $A$ is a unital $\mathrm{C}^{*}$-algebra $\bar{A}$ that contains $A$ as an essential ideal.

Definition 1.18. Given a non-unital $\mathrm{C}^{*}$-algebra $A$, a unitization $\bar{A}$ defines a noncommutative coarse structure on $A$.

To make more precise the condition of containing $A$ as an ideal, we choose a particular representation of $A$ inside $B(H)$, the bounded operators on some Hilbert space $H$. Then the unitization $\bar{A}$ is a unital $\mathrm{C}^{*}$-algebra sitting inside $B(H)$. More concretely, we need to understand the Gelfand-Naimark Theorem of representing abstract $\mathrm{C}^{*}$-algebras isometrically as $\mathrm{C}^{*}$-subalgebras of $B(H)$. 


\subsubsection{The Gelfand-Naimark correspondence}

Next we give a rapid and brief introduction to several $\mathrm{C}^{*}$-algebraic concepts we need to establish the Gelfand-Naimark Theorem.

Definition 1.19. Let $A$ be a $\mathrm{C}^{*}$-algebra and let $H$ be a Hilbert space. A representation $\pi$ of $A$ on $H$ is a $*$-homomorphism $\pi: A \rightarrow B(H)$. In other words, $\pi$ is a linear map from $A$ to $B(H)$ such that

$$
\pi(a b)=\pi(a) \pi(b) \quad \text { and } \quad \pi\left(a^{*}\right)=\pi(a)^{*}
$$

for all $a, b \in A$. A representation is called faithful if it is injective.

Definition 1.20. A representation $\pi: A \rightarrow B(H)$ is called irreducible if $\pi(A)$ has no invariant subspaces other than 0 and $H$.

Definition 1.21. A vector $\eta \in H$ is called cyclic if the closed linear span of $\pi(A) \eta$, written as $[\pi(A) \eta]$, is the whole of $H$. A representation $\pi: A \rightarrow B(H)$ is cyclic if there exists a cyclic vector $\eta \in H$.

Definition 1.22. Bounded positive linear functions of norm 1 on a $\mathrm{C}^{*}$-algebra $A$ are called states of the algebra. Here the norm of a linear function $f: A \rightarrow \mathbb{C}$ is defined as

$$
\|f\|:=\sup _{\|x\|=1}|f(x)| .
$$

If $A$ is unital, then the norm of a positive linear functional is $\|f\|:=f(1)$, where 1 is the unit of $A$.

Example 1.23. In the case of $C(X)$, by the Riesz Representation Theorem the space of states consists of all regular Borel probability measures on $X$.

This is a convex space and the elements $x \in X$ correspond to the extreme points of the state space. The notion of extreme points of a convex subset of a topological vector space is defined as those which do not have a non-trivial convex decomposition. Thus the multiplicative linear functionals are just the extreme points in the space of states on $C(X)$.

Definition 1.24. A pure state is an extreme point in the state space of a $\mathrm{C}^{*}$-algebra $A$.

Given a $\mathrm{C}^{*}$-algebra $A$, consider a state $\phi: A \rightarrow \mathbb{C}$. Then by the standard Gelfand-Naimark-Segal construction in $\mathrm{C}^{*}$-algebra theory, $\phi$ corresponds to a cyclic representation of $A$, say, $\left(\pi_{\phi}, H_{\phi}, \zeta_{\phi}\right)$. See [6. Theorem 3.3.3] for the construction.

If $\phi$ is pure then the representation $\pi_{\phi}$ is irreducible. Thus each pure state corresponds to an irreducible representation of the $\mathrm{C}^{*}$-algebra. In the converse direction, every irreducible representation is spatially equivalent to a cyclic representation corresponding to a pure state. Spatial equivalence is a very natural equivalence relation between representations of a $\mathrm{C}^{*}$-algebra.

Definition 1.25. A representation $\pi_{1}: A \rightarrow B\left(H_{1}\right)$ is said to be spatially equivalent to $\pi_{2}: A \rightarrow B\left(H_{2}\right)$ iff there exists a unitary $U: H_{1} \rightarrow H_{2}$ such that $U \pi_{1}(a)=\pi_{2}(a) U$ for all $a \in A$. 
Definition 1.26. The spectrum of a $\mathrm{C}^{*}$-algebra $A$ is defined as the set of spatial equivalence classes of irreducible representations of $A$.

In the commutative case, there is a one-to-one correspondence between the set of pure states and the spectrum of the $\mathrm{C}^{*}$-algebra, that is, $P(A)=\hat{A}$. For a commutative $\mathrm{C}^{*}$-algebra, the irreducible representations are all one-dimensional, that is, $*$-homomorphisms $\phi: A \rightarrow \mathbb{C}$. Hence the equivalence relation is trivial. Thus there is only one element in each spatial equivalence class of irreducible representations, namely, the pure state $\phi$.

In a commutative $\mathrm{C}^{*}$-algebra $A$, for every element $a$ in $A$ one can define a function over its pure state space by

$$
\hat{a}: \phi \mapsto \phi(a) ; \quad \forall \phi \in P(A) .
$$

This is known as the Gelfand transform and defines a homomorphism from $A$ to $C(P(A))$.

Theorem 1.27 (Gelfand-Naimark). Let $A$ be a commutative unital $C^{*}$-algebra with spectrum $\hat{A}=P(A)$. The Gelfand transform is an isometric $*$-isomorphism of $A$ onto $C(\hat{A})$.

For a noncommutative $\mathrm{C}^{*}$-algebra $A$, corresponding to each self-adjoint element $a \in A_{\mathrm{sa}}$, there exists a pure state $f$ on $A$ such that $|f(a)|=\|a\|_{A}$. Then for every $a \in A$, considering the positive element $a^{*} a$, we have the GNS representation $\pi_{a}$ such that $\left\|\pi_{a}(a)\right\|_{B\left(H_{a}\right)}=\|a\|_{A}$. One can then consider the direct sum of representations $\bigoplus_{a \in A} \pi_{a}$ which shall give an isometric $*$-representation of $A$, as in the following theorem.

Theorem 1.28 (Gelfand-Naimark). Every abstract $C^{*}$-algebra with identity is isometrically $*$-isomorphic to a $C^{*}$-algebra of operators on some Hilbert space.

For a noncommutative $\mathrm{C}^{*}$-algebra, the space of pure states is mostly larger than $\hat{A}$ and there exists a surjection from $P(A)$ onto the spectrum $\hat{A}$. For example, the spectrum of the algebra of compact operators, which is isomorphic to the Moyal plane, consists of only one point. This is why we avoid a set-theoretic definition of coarse structure on the underlying topological space, unless we find more reasons to choose $P(A)$ over $\hat{A}$. Even then, with either of the choices, there are other limitations with their topology for noncommutative $\mathrm{C}^{*}$-algebras.

\subsubsection{Compactifications and unitizations}

In this section, we look into examples of compactifications of a locally compact Hausdorff space. We shall then consider corresponding examples of unitizations of $\mathrm{C}^{*}$-algebras.

The partial order in the collection of all compactifications of a given locally compact Hausdorff space $X$ is given as $X^{1} \leqslant X^{2}$ iff there exists a surjective quotient map $q: X^{2} \rightarrow X^{1}$. Consider the partially ordered set of all possible compactifications for a given locally compact Hausdorff space $X$. The one-point compactification $X^{\dagger}$ of $X$ is the smallest possible compactification. It is defined 
as the original space $X$ along with an extra point, say $x_{\infty}$, that is, $X^{\dagger}:=X \cup x_{\infty}$. To define the topology of $X^{\dagger}$ we define the neighbourhoods of the point $x_{\infty}$ as the complements of compact sets in $X$. These extra open sets along with the original topology of $X$ define the new compact topological space $X^{\dagger}$. If $X$ is already compact then the point $x_{\infty}$ will be an isolated point of $X^{\dagger}$ and $X^{\dagger}$ is not a compactification of $X$.

Example 1.29. Consider the locally compact Hausdorff space $\mathbb{R}^{n}$. The onepoint compactification of $\mathbb{R}^{n}$ is homeomorphic to the sphere $S^{n}$. Thinking of the compactification process as attaching some boundary to $\mathbb{R}^{n}$ the one-point compactification can be thought of as the spherical completion of $\mathbb{R}^{n}$.

The Stone-Čech compactification is the largest possible compactification of $X$. It is defined as a compact Hausdorff space $\beta X$ containing $X$ as a dense subset such that all continuous bounded function on $X$ have a unique extension to $\beta X$. It is the spectrum of $C_{b}(X)$.

The Stone-Čech compactification $\beta X$ is the maximal compactification in the sense that any other compactification is a quotient of $\beta X$. The one-point compactification $X^{\dagger}$ is the smallest, so all other compactifications of $X$ map onto it.

Example 1.30. One can also think of other compactifications of $\mathbb{R}^{n}$, for example, like the projective completion. This would start from the centre and follow a ray up until infinity and if we continue further beyond that we come back along the line of the ray from the diametrically opposite point to the centre. The unital algebra of continuous functions on the projective compactification thus contain all bounded functions that descends down to the projective space $\mathbb{P R}^{n}$.

Using the Gelfand-Naimark correspondence, for a non-unital $\mathrm{C}^{*}$-algebra $A$ we expect a similar partially ordered class of unitizations of $A$. Given $A$, we can always define a minimal unitization by just adjoining a unit to $A$.

The minimal unitization $A^{\dagger}$ of $A$ is isomorphic to the direct sum $A \oplus \mathbb{C}$ as a Banach space with the multiplication given by $\left(a, \lambda_{1}\right) *\left(b, \lambda_{2}\right)=\left(a \cdot b+\lambda_{2} a+\right.$ $\left.\lambda_{1} b, \lambda_{1} \lambda_{2}\right) . A^{\dagger}$ acts on $A$ with this multiplication. The embedding of $A$ into $A^{\dagger}$ is explicitly given by $a \mapsto(a, 0)$; for all $a \in A$. Thus we define a $\mathrm{C}^{*}$-operator norm on the elements of $A^{\dagger}$ by $\|(a, \lambda)\|=\sup _{b \in A,\|b\|<1}\|(a, \lambda) *(b, 0)\|$. One can show that this gives a unital $\mathrm{C}^{*}$-algebra containing $A$ as an essential ideal with the unit element given by $(0,1)$. If $A$ already contains a unit, then $A^{\dagger} \cong A \oplus \mathbb{d}^{6}$ with multiplication $\left(a, \lambda_{1}\right) *\left(b, \lambda_{2}\right)=\left(a b, \lambda_{1} \lambda_{2}\right)$.

The multiplier algebra $M(A)$ is the largest unitization of $A$. In the commutative case, the multiplier algebra of $C_{0}(X)$ is the algebra of all continuous bounded functions $C_{b}(X)$ on $X$. If we want to look for a concrete realization of $M(A)$, we shall have to extend a representation of $A$ as bounded operators on some Hilbert space $B(H)$ to the bigger algebra $M(A)$. For this, we need a non-degenerate representation $\pi: A \rightarrow B(H)$, that is, $\pi(A) H$ is dense in $H$.

Definition 1.31. Given a non-degenerate representation of $A$ in $B(H)$, an element $x \in B(H)$ is called a two-sided multiplier of $A$ if $A x \subset A$ and $x A \subset A$.

\footnotetext{
${ }^{6}$ Please note that as in the commutative case, $A^{\dagger}$ is a unitization of $A$ only if $A$ is non-unital.
} 
The set containing all these elements is called the multiplier algebra for $A$ :

$$
M(A):=\{x \in B(H) \mid x A \subset A \wedge A x \subset A\}
$$

But as we expect from the commutative case, the notion of unitization is independent of the concrete representation of the algebra. To see that the definition above is independent of the chosen nondegenerate representation of $A$, we map this to an algebra whose definition is independent of the representation of $A$. This is the algebra of double centralizers of $A$.

Definition 1.32. A double centralizer of a $\mathrm{C}^{*}$-algebra $A$ is a pair of maps $(L, R): A \rightarrow A$ satisfying

$$
R(x) y=x L(y)
$$

for all $x, y$ in $A$. Let us denote the set of all double centralizers of $A$ as $\operatorname{DC}(A)$.

The unit in $\operatorname{DC}(A)$ is the pair $(I, I)$, where $I$ is the identity map on $A$. If $T: A \rightarrow A$ is any map, let $T^{*}: A \rightarrow A$ be the map $a \mapsto\left(T\left(a^{*}\right)\right)^{*}$, and define

$$
(L, R)^{*}:=\left(R^{*}, L^{*}\right) .
$$

Then * is an involution in $\operatorname{DC}(A)$, which makes $\operatorname{DC}(A)$ a unital *-algebra. By using the algebraic conditions, the elements of $\operatorname{DC}(A)$ can be given a unique operator $\mathrm{C}^{*}$-norm with which $\mathrm{DC}(A)$ is a unital $\mathrm{C}^{*}$-algebra containing $A$. The motivation for looking at the set of all double centralizers of $A$ as the multiplier algebra can be seen from the following example.

Example 1.33. If $A$ is an ideal in the $\mathrm{C}^{*}$-algebra $B$ and $x \in B$, then

$$
L_{x}: a \mapsto x a ; \quad R_{x}: a \mapsto a x
$$

are maps from $A$ to $A$. Since $a L_{x}(b)=a x b=R_{x}(a) b$ for all $a, b \in A,\left(L_{x}, R_{x}\right)$ is a double centralizer for $A$. The map $x \mapsto\left(L_{x}, R_{x}\right)$ between $M(A)$ and $\operatorname{DC}(A)$ is an isomorphism of $\mathrm{C}^{*}$-algebras. The algebra $\mathrm{DC}(A)$ is independent of choices.

Thus starting with an abstract $\mathrm{C}^{*}$-algebra $A$ we can construct its multiplier algebra $M(A)$. If $A$ is already unital, then $M(A)=A$. This characterization of the biggest unitization $M(A)$ of $A$ independent of the representation of $A$ on some $B(H)$ helps us to define other possible unitizations abstractly as subalgebras of $M(A)$.

Definition 1.34. A unital $\mathrm{C}^{*}$-subalgebra $B$ of $M(A)$ containing $A$ is called a unitization of $A$.

\subsection{Boundaries of compactifications}

For topological coarse structures, the boundary of the compactification is an important invariant of the coarse structure. In fact, as we saw, the controlled sets are directly defined in terms of convergence properties of nets as they approach the boundary. In the last chapter, we shall see that coarse maps, the 
appropriate morphisms in the coarse category, between topological coarse structures are also determined by their behaviour at the boundary. Thus we end this chapter with a brief study of topological boundaries and the boundary quotient algebras corresponding to unitizations of $\mathrm{C}^{*}$-algebras.

Definition 1.35. Given an ideal $J$ in a $\mathrm{C}^{*}$-algebra $A$, the quotient algebra $A / J$ becomes a $\mathrm{C}^{*}$-algebra with the following quotient norm

$$
\|a+J\|:=\inf \{\|a+j\|: \forall j \in J\} .
$$

Given a unitization $\bar{A}$ of a non-unital $\mathrm{C}^{*}$-algebra $A, A$ being an ideal in $\bar{A}$, the boundary quotient algebra $\bar{A} / A$ is a $\mathrm{C}^{*}$-algebra with the quotient norm.

In particular, for certain noncommutative $\mathrm{C}^{*}$-algebras $A$ and their unitizations $\bar{A}$ the boundary quotient algebra $\bar{A} / A$ can be shown to be $*$-isomorphic to the commutative $\mathrm{C}^{*}$-algebra $C(\bar{X}) / C_{0}(X)$, which is the boundary quotient algebra for some locally compact Hausdorff topological space $X$ with compactification $\bar{X}$. The Stone-Weierstrass Theorem implies the following result.

Lemma 1.36. For a locally compact Hausdorff space $X$ with compactification $\bar{X}$, the commutative $C^{*}$-algebra $C(\bar{X}) / C_{0}(X)$ is naturally isomorphic to $C(\partial \bar{X})$. Here $\partial \bar{X}$ is the boundary $\overline{\bar{X}} \backslash X$ of the compactification $\bar{X}$.

Therefore, extending the isomorphism of $\mathrm{C}^{*}$-algebras $\bar{A} / A \cong C(\bar{X}) / C_{0}(X)$ to $\bar{A} / A \cong C(\partial \bar{X})$, we have the noncommutative coarse structure on $A$ as coming from the commutative boundary $\partial \bar{X}$. Depending on the strength of the boundary with respect to determining a unique underlying noncommutative coarse structure, we can use established notions from topological coarse structures to better understand the noncommutative coarse structure on $A$ coming from $\bar{A}$. Our example of a coarse equivalence between the Moyal plane and the classical plane is based on similar ideas.

The boundary of the Higson compactification of a proper coarse space is called Higson corona. As we shall see in Chapter 3, Proposition 3.28, the Higson corona is functorial from the category of proper coarse spaces with coarse maps as morphisms to that of compact topological spaces with continuous maps between them. For this, the coarse map need not be continuous.

In the next chapter, we consider examples of noncommutative coarse structures on $\mathrm{C}^{*}$-algebras by using the method of Rieffel deformation. In the last chapter, we shall see that the functoriality of the Higson corona plays a major role to imply coarse equivalence of the metric coarse structure on $\mathbb{R}^{2 n}$ with that on the Moyal plane.

\footnotetext{
${ }^{7}$ For the boundary to be compact, we need $\bar{X}$ to be Hausdorff, and hence $X$ should also be Hausdorff.
} 


\section{Chapter 2}

\section{Noncommutative coarse structures}

The main objective of this chapter is to outline the construction of new noncommutative coarse structures from given ones. Rieffel deformation of $\mathrm{C}^{*}$-algebras by actions of the vector group $\mathbb{R}^{d} \mid 7$ helps us to construct such noncommutative coarse structures. More generally, Rieffel deformation generalized to deformation of $\mathrm{C}^{*}$-algebras by 2-cocycles on the dual group of a locally compact abelian group, as developed by Kasprzak [4], also provides us with similar constructions.

The deformation procedure strongly depends on the action of the group on the $\mathrm{C}^{*}$-algebra. Given a noncommutative coarse structure on a $\mathrm{C}^{*}$-algebra $A$ by a unitization $\bar{A}$, we require the group actions to give an equivariant embedding of $A$ into $\bar{A}$. With this, the group action then descends to an action on the boundary quotient algebra $\bar{A} / A$. This then gives a $G$-equivariant exact sequence

$$
0 \rightarrow A \rightarrow \bar{A} \rightarrow \bar{A} / A \rightarrow 0 .
$$

Since the deformation procedure under a 2-cocycle $\Psi$ preserves exactness, this gives a coarse structure on $A^{\Psi}$, given by the extension

$$
0 \rightarrow A^{\Psi} \rightarrow \bar{A}^{\Psi} \rightarrow(\bar{A} / A)^{\Psi} \rightarrow 0 .
$$

We apply this, in particular, to the unitization of $C_{0}\left(\mathbb{R}^{2 n}\right)$ coming from the Higson compactification for the usual Euclidean metric on $\mathbb{R}^{2 n}$. Rieffel deformation gives a coarse structure on the Moyal plane. As we shall see in the last chapter, this coarse structure is coarsely equivalent to the classical plane $\mathbb{R}^{2 n}$.

\section{$2.1 \quad$ Rieffel deformation}

Rieffel in his work 7 develops the theory of deformations of a $\mathrm{C}^{*}$-algebra $A$ with a strongly continuous action $\alpha$ of the vector group $V:=\mathbb{R}^{d}$. The main aim of this deformation is to arrive at a deformed product, examples of which 
would be the Moyal product and the general theory of deformation quantization that was being considered mostly in physics. In deformation quantization, the deformed product is defined on the dense smooth subalgebra and then extended by continuity. Similarly, one can study deformed products on $\mathrm{C}^{*}$-algebras admitting an action of the vector group $V$.

The formula for the deformed product is written down concretely for elements of the subalgebra $\mathcal{A} \subset A$ that are smooth with respect to the action. Let $J$ be an anti-symmetric matrix on $V$. The Rieffel deformation $\mathcal{A}_{J}$ of $\mathcal{A}$ is the same Fréchet space $\mathcal{A}$ equipped with the new product

$$
a \times_{J} b:=\int_{V \times V} \alpha_{J x}(a) \alpha_{y}(b) e(x \cdot y) \mathrm{d} x \mathrm{~d} y{ }_{1}^{1}
$$

where $e(x \cdot y)$ stands for $e^{2 \pi i\langle x, y\rangle}$ for a given inner product $\langle$,$\rangle on the vector$ group $V$.

Rieffel also constructs certain $\mathrm{C}^{*}$-norms on $\mathcal{A}_{J}$, which involve representing $\mathcal{A}_{J}$ by adjointable operators on a pre-Hilbert $A$-module 2 With this $\mathrm{C}^{*}$-norm, the smooth algebra with the deformed product is then completed and is known as the Rieffel deformation $A_{J}$ of the $\mathrm{C}^{*}$-algebra $A$. In the special case of $A \cong C_{0}\left(\mathbb{R}^{2 n}\right)$, with translation action by the vector group $\mathbb{R}^{2 n}$, the deformed $\mathrm{C}^{*}$-algebra corresponds to the Moyal plane, one of our major motivating examples for studying noncommutative coarse structures.

As we see, the set-up automatically involves the data of a $\mathrm{C}^{*}$-dynamical system, which we define below.

Definition 2.1. A $\mathbf{C}^{*}$-dynamical system $(A, G, \alpha)$ consists of a $\mathrm{C}^{*}$-algebra $A$ with a strongly continuous action $\alpha$ of a locally compact abeliar ${ }^{3}$ group $G$ on the $\mathrm{C}^{*}$-algebra $A$.

Remark 2.2. In a $\mathrm{C}^{*}$-dynamical system $(A, G, \alpha)$, the group action $\alpha$ of $G$ on $A$ being strongly continuous refers to a continuous homomorphism of $G$ into the group $\operatorname{Aut}(A)$ of *-automorphisms of $A$, equipped with the topology of pointwise convergence ${ }^{4}$ Strong continuity is a weaker condition than requiring the map $\alpha: G \times A \rightarrow A,(g, a) \mapsto \alpha_{g}(a)$ to be jointly continuous with respect to the topologies of $G$ and $A$.

Given a $\mathrm{C}^{*}$-dynamical system as above, each element $a$ in $A$ can be seen as a bounded continuous function in $C_{b}(G ; A)$, given by $g \mapsto \alpha_{g}(a)$. This map $\tilde{\alpha}: A \rightarrow C_{b}(G ; A)$ is then a continuous *-homomorphism equivariant for the action $\alpha$ on $A$ and the translation action $\tau$ on $C_{b}(G ; A)$. Now for the vector group $V$, with differentiation being allowed, by Proposition 2.10 in $[7$, the map above shall carry the smooth elements $\mathcal{A} \subset A$ with respect to the action $\alpha$ to smooth functions inside $C_{b}(V ; A)$. Rieffel then develops the deformed product

\footnotetext{
${ }^{1}$ For the standard symplectic matrix $J$ this form of the oscillatory integral is equivalent to $\int_{V \times V} \alpha_{x}(a) \alpha_{y}(b) e(x \cdot J y) \mathrm{d} x \mathrm{~d} y$, which is the familiar "Moyal product" in physics.

${ }^{2}$ See Definition 3.3 in the next chapter.

${ }^{3}$ We only consider abelian groups here.

${ }^{4}$ This means that for each $x$ in $A$, the function $\alpha(x): G \rightarrow A$ defined by $t \mapsto \alpha_{t}(x)$ is continuous in the norm topology of $A$.
} 
on these function algebras by defining a Moyal type product on the smooth functions and then extending it to the completion.

The algebra $C_{b}(V ; \mathcal{A})$ of bounded continuous functions on $V$ with coefficients in the smooth subalgebra $\mathcal{A}$ is also equipped with the (isometric) translation action of the vector group $V$. The subalgebra of uniformly continuous functions $C_{u}(V ; \mathcal{A}) \subset C_{b}(V ; \mathcal{A})$ is the largest subspace on which the translation action is strongly continuous. Following the same procedure with an isometric and strongly continuous action, we let $C_{u}^{\infty}(V ; \mathcal{A})$ denote the smooth subalgebra of infinitely differentiable functions which, together with all their derivatives of all orders, are bounded. It is trivial to see that, for $a$ in $\mathcal{A}$, the image $\tilde{\alpha}(a)$ under the map $\tilde{\alpha}$ above belongs to $C_{u}^{\infty}(V ; \mathcal{A})$. For functions $f, g$ in $C_{u}^{\infty}(V ; \mathcal{A})$ the deformed product is defined by

$$
\left(f \times{ }_{J} g\right)(x)=\int_{V \times V} f(x+J u) g(x+v) e(u \cdot v) \mathrm{d} u \mathrm{~d} v
$$

This is equivalent to 2.1, if we look at the elements $a$ in $\mathcal{A}$ as functions given by $\tilde{\alpha}(a){ }^{5}$

Next we look at its representation as adjointable operators on $\mathcal{S}(V ; \mathcal{A})$, the space of Schwartz functions from $V$ to $\mathcal{A}$, which form a pre-Hilbert $\mathcal{A}$-module.

Finally, we shall complete these representations to arrive at representations on the Hilbert $A$-module $L^{2}(V) \otimes A$. Then, with the corresponding $\mathrm{C}^{*}$-norm as adjointable operators, we complete the smooth algebras to arrive at the required $\mathrm{C}^{*}$-algebra $A$ and its Rieffel deformation $A_{J}$, respectively.

For the smooth Fréchet algebra $\mathcal{A} \subset A$, consider the following representation of $a$ in $\mathcal{A}$ on a function $\xi$ belonging to the Schwartz algebra $\mathcal{S}(V ; \mathcal{A})$ as

$$
(\pi(a) \xi)(x)=\alpha_{-x}(a) \xi(x),
$$

where $x$ is an element of the vector group $V$.

To arrive at the Hilbert $A$-module $L^{2}(V) \otimes A$ consider the following inner product on the algebra $\mathcal{S}(V ; \mathcal{A})$,

$$
\langle f, g\rangle=\int_{V} f(v)^{*} g(v) \mathrm{d} v
$$

This makes $\mathcal{S}(V ; \mathcal{A})$ into a pre-Hilbert right $A$-module with the norm on $\mathcal{S}(V ; \mathcal{A})$ given by

$$
\|f\|_{\mathcal{S}(V ; \mathcal{A})}:=\sqrt{\|\langle f, f\rangle\|_{A}} .
$$

The completion of $\mathcal{S}(V ; \mathcal{A})$ with respect to the norm above can be shown to be the right Hilbert $A$-module $L^{2}(V) \otimes A$.

Using the norm on $\mathcal{S}(V ; \mathcal{A})$ as above, the following is shown in [7]:

\footnotetext{
${ }^{5}$ This is the idea used by Rieffel, using the conditions of an isometric and strongly continuous action $\alpha$ on the Fréchet subspace $\mathcal{A}$, to extend the "Moyal product" to arbitrary $\mathrm{C}^{*}$-algebras $A$, admitting actions of $V$.
} 
Lemma 2.3. For every $f$ in $C_{u}^{\infty}(V ; \mathcal{A})$, the deformed product with Schwartz functions defines the following operator

$$
\begin{aligned}
f \times_{J} \cdot: \mathcal{S}(V ; \mathcal{A}) & \rightarrow \mathcal{S}(V ; \mathcal{A}), \\
g & \mapsto f \times_{J} g .
\end{aligned}
$$

This map is continuous with respect to $\|\cdot\|_{\mathcal{S}}$ and adjointable with adjoint $f^{*} \times{ }_{J} \cdot$

Now, for $\mathcal{A}$ with the deformed product, we also have a representation on the same space $\mathcal{S}(V ; \mathcal{A})$. This is given by $\left(\pi_{J}(a) \xi\right)(x)=\left(\alpha(a) \times_{J} \xi\right)(x)$, where we refer to " $\times J$ " as the deformed product with elements of the Schwartz algebra $\mathcal{S}(V ; \mathcal{A})$ as defined above. For $a \in \mathcal{A}$, the element $\alpha(a)$ is defined as the function $x \mapsto \alpha_{-x}(a)$ on the vector group $V$. For this construction to work, it is important that the product $\alpha(a) \times{ }_{J} \xi$ belongs to $\mathcal{S}(V ; \mathcal{A})$.

Consider the Fréchet algebra $C_{u}^{\infty}(V ; \mathcal{A})$ as above. Now the given action $\alpha$ being isometric, the function $\alpha(a)$ is uniformly continuous. Also $a$ being in the algebra of smooth elements $\mathcal{A}$, with respect to the action, $\alpha(a)$ shall also be a smooth function on $V$. Thus for a strongly continuous and isometric action $\alpha$ on the smooth elements $\mathcal{A}$, by Lemma $2.3 \alpha(a) \in C_{u}^{\infty}(V ; \mathcal{A})$.

The operator $\pi_{J}(a)$ can be written down in the form

$$
\begin{aligned}
\left(\pi_{J}(a) \xi\right)(x) & =\int_{V \times V} \alpha_{J y-x}(a) \xi(x-z) e(y \cdot z) \mathrm{d} y \mathrm{~d} z \\
& =\int_{V} \alpha_{J y-x}(a) \hat{\xi}(y) e(x \cdot y) \mathrm{d} y
\end{aligned}
$$

where for any $\xi$ in $\mathcal{S}(V ; \mathcal{A})$, by $[7$, Proposition 3.1$], \hat{\xi}$ in the second equality is the Fourier transform of $\xi$, given by

$$
\hat{\xi}(x)=\int_{V} \xi(y) \bar{e}(x \cdot y) \mathrm{d} y{ }^{6}
$$

With this, the representation $\pi_{J}$ of $\mathcal{A}_{J}$ on the pre-Hilbert module can be completed to a representation on the Hilbert $A$-module $L^{2}(V) \otimes A$. This then can be further extended to the completion of the algebra with respect to the $\mathrm{C}^{*}$-norm as adjointable operators on the Hilbert $A$-module.

Definition 2.4. The $\mathrm{C}^{*}$-algebra completion $A_{J}$ of $\mathcal{A}_{J}$ is the Rieffel deformation of $A$ under the action $\alpha$ of the vector group $V$, where $J$ is any antisymmetric matrix on $V$.

\subsubsection{Extending Rieffel deformation, crossed products}

One of the most noticeable point in the above consideration is that both the representations $\pi$ and $\pi_{J}$ are covariant with respect to the embedding of $V$ acting by left translation on $\mathcal{S}(V ; \mathcal{A})$. This automatically leads to considering the respective crossed product algebras.

\footnotetext{
${ }^{6} \bar{e}(x \cdot y)$ refers to the complex conjugate of $e(x \cdot y)$.
} 
Definition 2.5. Given representations $\pi$ of $A$ on a Hilbert space $H$ and a unitary representation $U$ of a group $G$ on $H$, then $(\pi, U, H)$ is a covariant representation of the $\mathrm{C}^{*}$-dynamical system $(A, G, \alpha)$ if

$$
\pi\left(\alpha_{x}(a)\right)=U_{x} \pi(a) U_{x}^{*}
$$

for all $a$ in $A$ and $x$ in $G$.

Definition 2.6. A crossed product for a $\mathrm{C}^{*}$-dynamical system $(A, G, \alpha)$ is a $\mathrm{C}^{*}$-algebra $B$ together with a non-degenerate $*$-homomorphism $i_{A}: A \rightarrow M(B)$ and a strictly continuous homomorphism $i_{G}: G \rightarrow U M(B)$ satisfying

1. The covariance condition $i_{A}\left(\alpha_{s}(a)\right)=i_{G}(s) i_{A}(a) i_{G}(s)^{*}$ for $a \in A, s \in G$.

2. For every covariant representation $(\pi, U)$ of $(A, G, \alpha)$, there is a nondegenerate representation $\pi \times U$ of $B$ with $\pi=(\pi \times U) \circ i_{A}$ and $U=$ $(\pi \times U) \circ i_{G}$.

3. The span of $\left\{i_{A}(a) i_{G}(z): a \in A, z \in C_{c}(G)\right\}$ is dense in $B$, where $i_{G}$ has been extended to the algebra $C_{c}(G)$ using integration over the group $G$.

Now, the representations $\pi, \pi_{J}, \lambda$, as considered in the previous section, are all on the space $\mathcal{S}(V ; \mathcal{A})$. The elements of the vector group $V$ are represented by left translation on $\mathcal{S}(V ; \mathcal{A})$ as $\lambda_{x} \otimes 1$, that is, for $\xi \in \mathcal{S}(V ; \mathcal{A})$,

$$
\left(\left(\lambda_{x} \otimes 1\right) \xi\right)(y)=\xi(y-x) .
$$

Then the following covariance relations hold:

$$
\begin{array}{r}
\left(\lambda_{x} \otimes 1\right) \pi(a)\left(\lambda_{-x} \otimes 1\right)=\pi\left(\alpha_{x}(a)\right) \\
\left(\lambda_{x} \otimes 1\right) \pi_{J}(a)\left(\lambda_{-x} \otimes 1\right)=\pi_{J}\left(\alpha_{x}^{J}(a)\right)
\end{array}
$$

For better clarity, let us prove the second equality.

Lemma 2.7. $\left(\lambda_{x} \otimes 1\right) \pi_{J}(a)\left(\lambda_{-x} \otimes 1\right)=\pi_{J}\left(\alpha_{x}^{J}(a)\right)$

Proof. For any $\xi$ in $\mathcal{S}(V ; \mathcal{A})$, consider the left hand side of the above equality,

$$
\begin{aligned}
& \left(\lambda_{x} \otimes 1\right) \pi_{J}(a)\left(\lambda_{-x} \otimes 1\right) \xi(w) \\
& =\left(\pi_{J}(a)\left(\lambda_{-x} \otimes 1\right) \xi\right)(w-x) \\
& =\pi_{J}(a)\left(\left(\lambda_{-x} \otimes 1\right) \xi\right)(w-x) \\
& =\alpha(a) \times_{J}\left(\left(\lambda_{-x} \otimes 1\right) \xi\right)(w-x) \\
& =\int_{V} \alpha_{J y-(w-x)}(a)\left[\left(\lambda_{-x} \otimes 1\right) \xi\right](y) e((w-x) \cdot y) \mathrm{d} y \\
& =\int_{V} \alpha_{J y-(w-x)}(a) e(x \cdot y) \hat{\xi}(y) e(w \cdot y) e((-x) \cdot y) \mathrm{d} y \\
& =\int_{V} \alpha_{J y-(w-x)}(a) \hat{\xi}(y) e(w \cdot y) \mathrm{d} y
\end{aligned}
$$


The right hand side of the above equality is,

$$
\begin{aligned}
& \pi_{J}\left(\alpha_{x}^{J}(a)\right) \xi(w) \\
& =\left(\alpha_{x}^{J}(a) \times_{J} \xi\right)(w) ; \alpha_{x}^{J}(a)=\alpha_{x}(a) \forall a \in \mathcal{A}^{J}=\mathcal{A} \\
& =\int_{V} \alpha_{J y-w+x}(a) \hat{\xi}(y) e(w \cdot y) \mathrm{d} y
\end{aligned}
$$

Using the covariance condition, let us define the convolution product in the algebra $\mathcal{S}(V ; \mathcal{A})$ to $\mathrm{b}]^{7}$

$$
(f * g)(x)=\int_{V} f(y) \alpha_{y}(g(x-y)) \mathrm{d} y .
$$

We shall refer to the algebra $\mathcal{S}(V ; \mathcal{A})$ with the above multiplication as the smooth crossed product $A \rtimes_{\alpha} V$. Similarly, for the covariant system consisting of $\left(\pi_{J}, \lambda, \alpha^{J}\right)$ for the deformed algebra $\mathcal{A}^{J}$, we shall consider $\mathcal{S}\left(V ; \mathcal{A}_{J}\right)$ with a similar convolution product as the smooth crossed product $\mathcal{A}_{J} \rtimes_{\alpha^{J}} V$.

The integrated forms of the representations $\pi, \pi_{J}$ on $\mathcal{S}(V ; \mathcal{A})$ are

$$
\begin{aligned}
\pi(f)(\xi)(x) & =\left(\int_{V} \pi(f(y))\left(\lambda_{y} \otimes 1\right) \mathrm{d} y\right)(\xi)(x) \\
& =\int_{V} \alpha_{-x}(f(y))\left(\left(\lambda_{y} \otimes 1\right) \xi\right)(x) \mathrm{d} y \\
& =\int_{V} \alpha_{-x}(f(y)) \xi(x-y) \mathrm{d} y
\end{aligned}
$$

and

$$
\begin{aligned}
\pi_{J}(f)(\xi)(x) & \left.=\int_{V}\left(\pi_{J}(f(z))\right)\left(\lambda_{z} \otimes 1\right) \xi\right)(x) \mathrm{d} z \\
& =\int_{V \times V} \alpha_{J y-x}(f(z))\left[\left(\widehat{\lambda_{z} \otimes 1}\right) \xi\right](y) e(x \cdot y) \mathrm{d} y \mathrm{~d} z \\
& =\int_{V \times V} \alpha_{J y-x}(f(z)) \hat{\xi}(y) e(-z \cdot y) e(x \cdot y) \mathrm{d} y \mathrm{~d} z \\
& =\int_{V} \alpha_{J y-x}(\hat{f}(y))(\hat{\xi}(y)) e(x \cdot y) \mathrm{d} y .
\end{aligned}
$$

Let us define the operator $\Theta_{J}$ on $\mathcal{S}(V ; \mathcal{A})$ as

$$
\Theta_{J}(f)(x)=\int_{V} \alpha_{J y}(\hat{f}(y)) e(x \cdot y) \mathrm{d} y .
$$

This operator is invertible, with inverse given by $\Theta_{-J}$.

Theorem 2.8. [5, Theorem 1.1] For every $f$ in $\mathcal{S}(V ; \mathcal{A})$ we have $\pi_{J}(f)=$ $\pi\left(\Theta_{J}(f)\right)$. Hence $\Theta_{J}$ defines an isomorphism $\mathcal{A} \rtimes_{\alpha} V \cong \mathcal{A}_{J} \rtimes_{\alpha^{J}} V$.

\footnotetext{
${ }^{7}$ This is the convolution product one uses in the compactly supported functions $C_{c}(V ; A)$ for a $\mathrm{C}^{*}$-algebra $A$ to arrive at the crossed product algebra $A \rtimes_{\alpha} V$. The algebra of Schwartz functions $\mathcal{S}(V ; \mathcal{A})$ is the completion of $C_{c}(V ; \mathcal{A})$ in a certain topology just as in the scalar case.
} 
Proof.

$$
\begin{aligned}
\left(\pi\left(\Theta_{J}(f)\right) \xi\right)(x) & =\int_{V} \alpha_{-x}\left(\Theta_{J}(f)(z)\right) \xi(x-z) \mathrm{d} z \\
& =\int_{V \times V} \alpha_{J y-x}(\hat{f}(y)) \xi(x-z) e(y \cdot z) \mathrm{d} y \mathrm{~d} z \\
& =\int_{V \times V} \alpha_{J y-x}(\hat{f}(y)) \xi(z) e(-y \cdot z) e(x \cdot y) \mathrm{d} y \mathrm{~d} z \\
& =\int_{V} \alpha_{J y-x}(\hat{f}(y)) \hat{\xi}(y) e(x \cdot y) \mathrm{d} y \\
& =\pi_{J}(f)(\xi)(x) .
\end{aligned}
$$

Since the smooth subalgebra, under a strongly continuous and isometric action, is dense, we may extend the whole picture to the level of $\mathrm{C}^{*}$-algebras. Firstly, complete the representations $\pi, \pi_{J}$ on $\mathcal{S}(V ; \mathcal{A})$ to that as adjointable operators on the Hilbert $A$-module $L^{2}(V) \otimes A$. Then using the $\mathrm{C}^{*}$-operator norm, the isomorphism $\Theta_{J}$ between the smooth crossed products extends to the $\mathrm{C}^{*}$-crossed product algebras:

Theorem 2.9. [5, Theorem 2.1] The isomorphism $\Theta_{J}$ of smooth crossed products extends to an isomorphism of the $C^{*}$-algebra crossed products $A_{J} \rtimes_{\alpha^{J}} V$ and $A \rtimes_{\alpha} V$.

\subsection{Rieffel deformation via crossed products}

Having established the crossed product isomorphisms between the original algebra and its Rieffel deformation, we shall next approach this from a different direction. Starting with the big $\mathrm{C}^{*}$-algebra of the crossed products, we shall extract the underlying data of the $\mathrm{C}^{*}$-dynamical system that is behind it. This is Landstad's approach to a $G$-product, [6. Section 7.8], and was explicitly used by Kasprzak in his approach to Rieffel deformation [4]. We shall set up next the framework required for it.

\subsubsection{G-product}

Let us start with the definition of a $G$-product, where $G$ is some locally compact abelian group. Let $\hat{G}$ be its Pontryagin dual.

Definition 2.10. Let $B$ be a $C^{*}$-algebra. Let $\lambda$ be a homomorphism of $G$ into the unitary group of $M(B)$ continuous in the strict topology of $M(B)$ and $\hat{\rho}$ a strongly continuous action of $\hat{G}$ on $B$. The triple $(B, \lambda, \hat{\rho})$ is called a $G$-product if

$$
\hat{\rho}_{\chi}\left(\lambda_{h}\right)=\langle\chi, h\rangle \lambda_{h}
$$

for all $\chi \in \hat{G}$ and $h \in G$. 
The unitary representation $\lambda: G \rightarrow M(B)$ can be extended to a morphism of $\mathrm{C}^{*}$-algebras $\lambda: C^{*}(G) \rightarrow M(B)$, where $C^{*}(G)$ is the group $\mathrm{C}^{*}$-algebra ${ }^{8}$ of $G$. From the isomorphism $C^{*}(G) \cong C_{0}(\hat{G})$, using the Fourier transform on the Hilbert spaces $\mathcal{F}: L^{2}(G) \rightarrow L^{2}(\hat{G})$, the map $\lambda$ gives a morphism $\lambda^{\prime}: C_{0}(\hat{G}) \rightarrow$ $M(B)$.

The map $\lambda^{\prime}$ is injective and hence is an embedding of the $\mathrm{C}^{*}$-algebra $C_{0}(\hat{G})$ into $M(B)$. Using the identity of the $G$-product 2.2 , we see that given the shift automorphism $\tau_{\chi} \in C_{0}(\hat{G})$ defined as

$$
\tau_{\chi}(f)\left(\chi^{\prime}\right)=f\left(\chi+\chi^{\prime}\right) \quad \forall f \in C_{0}(\hat{G}),
$$

the morphism $\lambda^{\prime}$ intertwines the action $\hat{\rho}$ with $\tau$ :

$$
\lambda^{\prime}\left(\tau_{\chi}(f)\right)=\hat{\rho}_{\chi}\left(\lambda^{\prime}(f)\right) .
$$

\subsubsection{Landstad algebra of a $G$-product}

Having defined the set-up of a $G$-product for a locally compact group $G$, we shall next apply it to understand Rieffel deformation using Landstad's approach to $G$-products. As mentioned in the beginning to this section, Landstad's theory is an alternative characterization of the crossed product algebra corresponding to a $\mathrm{C}^{*}$-dynamical system. We shall be able to show this only after we have understood the working of a certain fixed-point algebra, called the Landstad algebra, corresponding to such a framework.

Definition 2.11. Let $(B, \lambda, \hat{\rho})$ be a $G$-product and $x$ an element in $M(B)$. Then $x$ satisfies Landstad's conditions if the following holds:

1. $\hat{\rho}_{\chi}(x)=x$ for all $\chi \in \hat{G}$;

2. the map $G \ni g \mapsto \lambda_{g} x \lambda_{g}^{*} \in M(B)$ is norm continuous 9

3. $\lambda_{f} x, x \lambda_{g} \in B$ for all $f, g \in L^{1}(G)$ 10

The set of elements satisfying Landstad's conditions is a $\mathrm{C}^{*}$-algebra and is called the Landstad algebra of the $G$-product $(B, \lambda, \hat{\rho})$.

Remark 2.12. All crossed products $A \rtimes_{\alpha} G$ corresponding to a $\mathrm{C}^{*}$-dynamical system $(A, G, \alpha)$ are examples of a $G$-product. Given $A$, a $\mathrm{C}^{*}$-algebra, with a strongly continuous action $\alpha$ of $G$ on $A$, consider the standard dual action $\hat{\alpha}$ of the dual group $\hat{G}$ on $A \rtimes_{\alpha} G$. With the embedding of $G$ as unitary elements in $M\left(A \rtimes_{\alpha} G\right)$ given by $\lambda$, the triple $\left(A \rtimes_{\alpha} G, \lambda, \hat{\alpha}\right)$ is a $G$-product. In fact, we see below that all $G$-products are crossed products of the Landstad algebra $A$ with the action $\alpha$ implemented as conjugation by $\lambda$.

\footnotetext{
${ }^{8}$ Since $G$ is abelian, it is amenable and hence the reduced group $\mathrm{C}^{*}$-algebra is the same as the full group $\mathrm{C}^{*}$-algebra.

${ }^{9}$ This is equivalent to the action of the group $G$ in the underlying $\mathrm{C}^{*}$-dynamical system being strongly continuous.

${ }^{10}$ Given $f \in L^{1}(G)$, the element $\lambda_{f} \in M(B)$ is defined as

$$
\lambda_{f}=\int_{G} f(g) \lambda_{g} \mathrm{~d} g
$$
}


Theorem 2.13. 6. Theorem 7.8.8] A triple $(B, \lambda, \hat{\rho})$ is a G-product if and only if there is a $C^{*}$-dynamical system $(A, G, \alpha)$ such that $B=A \rtimes_{\alpha} G$ and $\hat{\rho}$ is the action $\hat{\alpha}$ of the dual group on the crossed product. This is unique up to isomorphism and $A$ consists of the elements in $M(B)$ that satisfy Landstad's conditions, with the action $\alpha_{g}(a)=\lambda_{g} a \lambda_{g}^{*}$.

\subsubsection{Deformation using a 2-cocycle on the dual group}

Now using the Landstad algebra corresponding to a $G$-product, we are going to recall the deformation procedure as developed by Kasprzak in [4]. A 2-cocycle on the dual group is what introduces the deformation, so let us start with it.

Definition 2.14. A 2-cocycle on the group $\hat{G}$ is a continuous function $\Psi: \hat{G} \times$ $\hat{G} \rightarrow \mathbb{T}^{1}$ satisfying

1. $\Psi(e, \chi)=\Psi(\chi, e)=1$ for all $\chi \in \hat{G}$

2. $\Psi\left(\chi_{1}, \chi_{2}+\chi_{3}\right) \Psi\left(\chi_{2}, \chi_{3}\right)=\Psi\left(\chi_{1}+\chi_{2}, \chi_{3}\right) \Psi\left(\chi_{1}, \chi_{2}\right)$ for all $\chi_{1}, \chi_{2}, \chi_{3} \in \hat{G}$.

The deformation procedure deforms the original dual action on the $\mathrm{C}^{*}$-algebra $B$ to another action on $B$. The second criterion says that this deformed dual action satisfies the identity 2.2 . For any $\chi, \chi^{\prime}$ in the dual group $\hat{G}$, consider the function $\Psi_{\chi}\left(\chi^{\prime}\right)=\Psi\left(\chi, \chi^{\prime}\right)$. This then defines a family of functions $\Psi_{\chi}: \hat{G} \rightarrow \mathbb{T}^{1}$, indexed by elements of the group $\hat{G}$. The functions $\Psi_{\chi}$ are only bounded continuous function on the group $\hat{G}$ and hence belong to $C_{b}(\hat{G})$. Using the Fourier transform, consider the isomorphism with the group $\mathrm{C}^{*}$-algebra $C^{*}(G) \cong C_{0}(\hat{G})$. Since the embedding of $C^{*}(G)$ in $M(B)$ is non-degenerate, we may extend the map $\lambda^{\prime}: C_{0}(\hat{G}) \rightarrow M(B)$ to the multiplier algebras. This gives a map $\overline{\lambda^{\prime}}: C_{b}(\hat{G}) \rightarrow M(B)$. The map $\bar{\lambda}^{\prime}$ is strictly continuous and hence gives a strictly continuous ${ }^{T}$ family of unitary elements

$$
U_{\chi}=\bar{\lambda}^{\prime}\left(\Psi_{\chi}\right) .
$$

The 2-cocycle condition for $\Psi$ then gives

$$
U_{\chi_{1}+\chi_{2}}=\overline{\Psi\left(\chi_{1}, \chi_{2}\right)} U_{\chi_{1}} \hat{\rho}_{\chi_{1}}\left(U_{\chi_{2}}\right) .
$$

Using this identity, one then constructs a deformed dual action as follows;

Theorem 2.15. [4, Theorem 3.1] Let $(B, \lambda, \hat{\rho})$ be a G-product and let $\Psi$ be a 2 -cocycle on $\hat{G}$. For any $\chi \in \hat{G}$, the map

$$
\hat{\rho}_{\chi}^{\Psi}: B \ni b \mapsto \hat{\rho}_{\chi}^{\Psi}(b)=U_{\chi}^{*} \hat{\rho}_{\chi}(b) U_{\chi} \in B
$$

is an automorphism of the $C^{*}$-algebra B. Moreover,

$$
\hat{\rho}^{\Psi}: \hat{G} \ni \chi \mapsto \hat{\rho}_{\chi}^{\Psi} \in \operatorname{Aut}(B)
$$

is a strongly continuous action of $\hat{G}$ on $B$ and the triple $\left(B, \lambda, \hat{\rho}^{\Psi}\right)$ is a $G$ product.

\footnotetext{
${ }^{11}$ Recall that the embedding $\lambda$ of $G$ in $M(B)$ was continuous in the strict topology of $M(B)$
} 
Let us denote the Landstad algebra of the new $G$-product $\left(B, \lambda, \hat{\rho}^{\Psi}\right)$ as $A^{\Psi}$, a subalgebra of $M(B)$. From the particular nature of the construction behind the deformation, the algebra $A^{\Psi}$ still carries an action of the group $G$ by

$$
\rho_{g}^{\Psi}(x)=\lambda_{g} x \lambda_{g}^{*} .
$$

Hence the $G$-product $\left(B, \lambda, \hat{\rho}^{\Psi}\right)$, defines a $C^{*}$-dynamical system $\left(A^{\Psi}, G, \rho^{\Psi}\right)$. By Theorem 2.13, the system is unique up to covariant isomorphisms, which gives the following result.

Proposition 2.16. [4. Proposition 3.2] Let $\left(A^{\Psi}, G, \rho^{\Psi}\right)$ be the dynamical system considered above. Then

$$
A \rtimes_{\rho} G \cong A^{\Psi} \rtimes_{\rho^{\Psi}} G .
$$

The Landstad algebra $A^{\Psi}$ under the deformed dual action, is the Rieffel deformation of $A$ corresponding to the 2-cocycle $\Psi$. Given an anti-symmetric matrix $J$ on the vector group $\hat{V}=V$, define the 2 -cocycle $\Psi_{J}(x, y):=e(x \cdot J y)$. We see next that $A^{\Psi_{J}}$ is the Rieffel deformation $A_{J}$ of $A$ corresponding to $J$, as in Definition 2.4. The construction for 2-cocycle deformation, as given above, coincides with that of the Rieffel deformation of $\mathrm{C}^{*}$-algebras by actions of the vector group $V$. Recall from our discussion before the representations of the original algebra $A$ along with the Rieffel deformation $A_{J}$ on the Hilbert $A$-module $L^{2}(V) \otimes A$. Also consider the explicit isomorphism $\Theta_{J}$ between the crossed products as in Theorem 2.9 above. Using $\pi \Theta_{J}=\pi_{J}$, we see that the map $a \mapsto(\mathcal{F} \otimes 1) \pi_{J}(a)\left(\mathcal{F}^{*} \otimes 1\right)$ defines an isomorphism $A_{J} \cong A^{\Psi_{J}}$.

Theorem 2.17. 5. Theorem 2.3] The map $\Theta_{J}: M\left(A_{J} \rtimes_{\alpha^{J}} V\right) \rightarrow M\left(A \rtimes_{\alpha} V\right)$ defines an isomorphism of the $C^{*}$-algebras $A_{J} \subset M\left(A_{J} \rtimes_{\alpha^{J}} V\right)$ and $\left(\mathcal{F}^{*} \otimes 1\right) A^{\Psi_{J}}(\mathcal{F} \otimes 1) \subset M\left(A \rtimes_{\alpha} V\right)$.

\subsubsection{The special case of a trivial action}

When the group action in the $\mathrm{C}^{*}$-dynamical system $(A, G, \alpha)$ is trivial, we claim that given any 2-cocycle, the above procedure of 2-cocycle deformation as constructed by Kasprzak, does not change the data of the $\mathrm{C}^{*}$-dynamical system. Namely, given $\alpha_{g}(a)=a, \forall g \in G, a \in A$, we show that for any given 2-cocycle $\Psi$ on the dual group $\hat{G}$, the deformed dynamical system $\left(A^{\Psi}, G, \alpha^{\Psi}\right)$ remains the same.

Let us look at the $G$-product $\left(A \rtimes_{\alpha} G, \lambda, \hat{\alpha}\right)$ corresponding to the $\mathrm{C}^{*}$-dynamical system $(A, G, \alpha)$ as above. Let $B=A \rtimes_{\alpha} G$. We know that the action $\alpha$ of the group $G$ on $A$ is given by the embedding $\lambda$ of $G$ in $M(B)$ by the following formula

$$
\alpha_{g}(a)=\lambda_{g} a \lambda_{g}^{*},
$$

where we use the standard nondegenerate embedding of $A$ in $M(B)$. Since the action $\alpha$ is trivial, the embeddings of $A$ and $G$ inside $M(B)$ commute with each other. That is, the following holds:

$$
\lambda_{g} a=a \lambda_{g} ; \quad \forall g \in G, a \in A
$$


This helps us to show that the deformed dual action $\hat{\alpha}^{\Psi}$ corresponding to the 2cocycle $\Psi$ on the dual group $\hat{G}$ acts trivially on the $\mathrm{C}^{*}$-algebra $A$. To show this, we need to show that $A$ commutes with the elements $U_{\chi}^{\Psi}$ as defined before, for all $\chi$ in $\hat{G}$. We have seen, that the elements $U_{\chi}^{\Psi}$ are the embedding of the functions $\Psi(\chi, \cdot) \in C_{b}(\hat{G})$ inside $M(B)$. The embedding uses the isomorphism with the group $\mathrm{C}^{*}$-algebra $C^{*}(G)$ and then extension of the nondegenerate embedding to the multiplier algebra. In fact, the image $\bar{\lambda}^{\prime}\left(C_{b}(\hat{G})\right)$ corresponds to the embedding of the commutative subalgebra $M\left(C^{*}(G)\right) \subset M(B)$.

Proposition 2.18. Under trivial action $\alpha$ of the group $G$ on $A$, the deformed dual action $\hat{\alpha}^{\Psi}$ is also trivial on the $C^{*}$-algebra $A$.

Proof. Recall the definition of the deformed action

$$
\hat{\alpha}_{\chi}^{\Psi}(a)=\left(U_{\chi}^{\Psi}\right)^{*} \hat{\alpha}_{\chi}(a) U_{\chi}^{\Psi} .
$$

Now, from equation 2.4 we see that $A$ commutes with $C^{*}(G)$ inside $M(B)$. Hence we expect it to commute also with the elements $U_{\chi}^{\Psi}$ inside $M(B)$, for all $\chi$ in $\hat{G}$. To see this, we first understand the definition of the unitaries $U_{\chi}^{\Psi}$ in terms of the map $\overline{\lambda^{\prime}}$. Using the Fourier transform $\mathcal{F}: L^{2}(G) \rightarrow L^{2}(\hat{G})$, we have the following definition of the map $\lambda^{\prime}$

$$
\lambda^{\prime}(f):=\lambda\left(\mathcal{F}^{*} f \mathcal{F}\right) ; \quad \forall f \in C_{0}(\hat{G}) .
$$

Next the strict continuity of $\lambda$ gives that $\lambda^{\prime}: C_{0}(\hat{G}) \rightarrow M(B)$ is a non-degenerate *-homomorphism. Hence it extends to the multiplier algebra $C_{b}(\hat{G})$ of $C_{0}(\hat{G})$. Conveniently, given an approximate unit12 $\left\{u_{\alpha}\right\}_{\alpha}$ in $C_{0}(\hat{G})$, the definition of the map $\bar{\lambda}^{\prime}: C_{b}(\hat{G}) \rightarrow M(B)$ can be given as

$$
\bar{\lambda}^{\prime}(f)=\lim _{\alpha} \lambda^{\prime}\left(f u_{\alpha}\right) ; \quad \forall f \in C_{b}(\hat{G}) .
$$

Then

$$
U_{\chi}^{\Psi}=\lim _{\alpha} \lambda^{\prime}\left(\Psi(\chi, \cdot) u_{\alpha}\right)
$$

Now, $A$ commutes with $C^{*}(G)$ and hence with $\lambda^{\prime}\left(\Psi(\chi, \cdot) u_{\alpha}\right)$ for all $\chi \in \hat{G}$ and all indices $\alpha$. Thus using equation 2.7 as the definition of $U_{\chi}^{\Psi}$ we see that the following equation

$$
\left(U_{\chi}^{\Psi}\right)^{*} a U_{\chi}^{\Psi}=a ; \quad \forall a \in A, \chi \in \hat{G}
$$

holds in the limiting sense of convergence in the strict topology of the multiplier algebra $M\left(C^{*}(G)\right)$ inside $M(B)$. But this topology being a locally convex topology on a vector space, is at least T0 ${ }^{13}$ and hence 2.8 actually holds. Next, we have already seen that $A$ being the Landstad algebra for $(B, \lambda, \hat{\alpha})$, is fixed by the undeformed dual action $\hat{\alpha}$. Therefore, in 2.5 , we have $\hat{\alpha}_{\chi}(a)=a$ for all $a$ in $A$ and $\chi$ in $\hat{G}$. Thus from equation 2.5 and 2.8 respectively, we see that the deformed dual action is trivial on the $\mathrm{C}^{*}$-algebra $A$.

\footnotetext{
${ }^{12}$ An approximate unit always converges strictly to 1 in $C_{b}(\hat{G})$.

${ }^{13} \mathrm{~T} 0$ refers to the separation axiom that given any two distinct points $x_{1} \neq x_{2}$ in the space, there exists a neighbourhood $V$ about $x_{2}$ such that $x_{1}$ does not belong to $V$. This is sufficient to claim that the constant net $\left\{\lambda^{\prime}\left(\Psi(\chi, \cdot) u_{\alpha}\right) a-a \lambda^{\prime}\left(\Psi(\chi, \cdot) u_{\alpha}\right)\right\}_{\alpha}=0$ converges to 0 .
} 
Remark 2.19. In the special case of trivial action $\alpha$, our goal is to show that the deformation procedure, using any 2-cocycle $\Psi$, does not change the $G$-product in any way. One way to see this would be that from the above Proposition 2.18, we know $A \subset A^{\Psi}$. Now, in the deformation procedure, we do not change the embedding of $G$ into $M(B)$. Therefore, we have the $\mathrm{C}^{*}$-dynamical system $(A, G, \alpha)$ contained in $\left(A^{\Psi}, G, \alpha^{\Psi}\right)$. But Proposition 2.16 states that $A \rtimes_{\alpha}$ $G \cong A^{\Psi} \rtimes_{\alpha^{\Psi}} G$, which imply $A=A^{\Psi}$, a standard result 6 , Proposition 7.7.9]. Hence for trivial actions, the deformed $G$-product, corresponding to any 2cocycle $\Psi$, is the same as the original $G$-product. Under a trivial action of the vector group $V$, a direct observation from the definition of the deformed product in Rieffel deformation gives a $*$-isomorphism $A \cong A^{J}$. Note that what we have shown a stronger result than just an isomorphism, and might prove useful for future developments of the treatment of noncommutative coarse geometry.

Next, we give another approach to the same result $A=A^{\Psi}$, under trivial action $\alpha$, by showing that the deformed dual action is the same as the original dual action on the whole crossed product algebra. What we have seen above, is that, for all $\chi$ in $\hat{G}$, the elements $U_{\chi}^{\Psi}$ commute with the embedding of $A$ in $M(B)$. Now, $M\left(C^{*}(G)\right)$ is already commutative. Thus the elements $U_{\chi}^{\Psi}$ commute both with the embedding of $A$ and $G$ in $M(B)$. With this, we claim that the deformed action of the dual group on $B$ is the same as the original dual action of $\hat{G}$ on $B$.

Proposition 2.20. For a $C^{*}$-dynamical system $(A, G, \alpha)$ with trivial action, the deformed dual action $\hat{\alpha}^{\Psi}$ on $B$, for any 2 -cocycle $\Psi$, is the same as $\hat{\alpha}$.

Proof. As we have seenn above, the deformed dual action is defined by

$$
\hat{\alpha}_{\chi}^{\Psi}(b)=U_{\chi}^{\Psi *} \hat{\alpha}_{\chi}(b) U_{\chi}^{\Psi} ; \quad \forall b \in B
$$

Now, by Proposition 2.18 $U_{\chi}^{\Psi}$ commutes with the embedding of $A$ in $M(B)$. Also, $G$ being abelian, $M\left(C^{*}(G)\right)$ is commutative and, therefore, $U_{\chi}^{\Psi}$ being contained in $M\left(C^{*}(G)\right)$, it commutes with all functions $z$ in $C_{c}(G)$. Now, the linear span of $K:=\left\{i_{A}(a) \lambda(z) \mid a \in A, z \in C_{c}(G)\right\} \sqrt{14}$ is dense in the crossed product $B$. Then $U_{\chi}^{\Psi}$ commutes with the elements of $K$ for all $\chi$ in $\hat{G}$ :

$$
U_{\chi}^{\Psi} i_{A}(a) \lambda(z)=i_{A}(a) U_{\chi}^{\Psi} \lambda(z)=i_{A}(a) \lambda(z) U_{\chi}^{\Psi} .
$$

Now, $U_{\chi}^{\Psi}$ being an element in the multiplier algebra of $B$, defines a continuous map from $B$ to itself by left or right multiplication with $U_{\chi}^{\Psi}$. Furthermore, $K$ is dense in $B$ in the norm topology. Hence $U_{\chi}^{\Psi}$ commuting with $K$ implies that $U_{\chi}^{\Psi}$ commutes with $B$ for all $\chi$ in $\hat{G}$. Hence, by definition, the deformed action $\hat{\alpha}^{\Psi}$ on $B$ is the same as $\hat{\alpha}$ for all 2-cocycles $\Psi$ on $\hat{G}$.

With this, we have the following two corollaries.

Corollary 2.21. For a trivial $C^{*}$-dynamical system $(A, G, \alpha)$, the deformed $G$ product $\left(B, \lambda, \hat{\alpha}^{\Psi}\right)$, corresponding to deformation by a 2-cocycle $\Psi$, is the same as the original $G$-product $(B, \lambda, \hat{\alpha})$.

\footnotetext{
${ }^{14}$ The embedding $\lambda$ of $G$ has been extended to $C_{c}(G)$ here.
} 
Corollary 2.22. For the trivial $C^{*}$-dynamical system $(A, G, \alpha)$, the deformed Landstad algebra $A^{\Psi}$ for any given 2 -cocycle $\Psi$ is same as $A$.

Therefore, given a trivial action of the group $G$ on $A$ we have the same $G$ product even after deformation. This is going to be important in defining a noncommutative coarse equivalence, as we shall see in the next chapter.

\subsection{Examples of noncommutative coarse struc- tures}

We use the theory of Rieffel deformation to define noncommutative coarse structures as outlined at the beginning of this chapter. Noncommutative coarse structures, coming from a commutative boundary quotient algebra, give us scope to compare with classical results in the theory of topological coarse structures. From this perspective, the best examples to study would be Rieffel deformation of commutative unital $\mathrm{C}^{*}$-algebras with trivial action on the boundary. We see that the approach via Landstad algebras, as in [4], give a more complete picture of Rieffel deformation useful for our purpose. It shows the embedding of the group algebra along with the deformed and the undeformed algebra. Hence we shall use Kasprzak's approach to Rieffel deformation from now on.

\subsubsection{Deformation procedure preserves exactness}

We shall see that, corresponding to a $G$-equivariant exact sequence of $G$-products, their Landstad algebras also form an exact sequence. Next we are going to use it to show that Rieffel deformation, or more generally a 2-cocycle deformation, preserves exactness.

Proposition 2.23. 4. Proposition 2.7] Let $(B, \lambda, \hat{\rho})$ and $\left(B^{\prime}, \lambda^{\prime}, \hat{\rho}^{\prime}\right)$ be $G$ products with $A, A^{\prime}$ as the respective Landstad algebras. Assume that, $\pi: B \rightarrow$ $M\left(B^{\prime}\right)$ is a non-degenerat $\Phi^{15}$ *-homomorphism satisfying the following conditions

- $\pi\left(\lambda_{g}\right)=\lambda_{g}^{\prime}$

- $\pi\left(\hat{\rho}_{\chi}(b)\right)=\hat{\rho}_{\chi}^{\prime}(\pi(b))$

Then

1. $\pi(A) \subset M\left(A^{\prime}\right)$.

2. if $\pi(B) \subset B^{\prime}$ then $\pi(A) \subset A^{\prime}$.

3. if $\pi(B)=B^{\prime}$ then $\pi(A)=A^{\prime}$

\footnotetext{
${ }^{15}$ In the applications, we shall have a unital $*$-homomorphism between unital $\mathrm{C}^{*}$-algebras
} 
It is important to note that the last condition suggests that surjectivity is preserved. An equivariant surjective map between $G$-C ${ }^{*}$-algebras $\varsigma^{16} \phi: A \rightarrow A^{\prime}$ shall induce a surjective map between the crossed product algebras $\phi: A \rtimes G \rightarrow A^{\prime} \rtimes G$, as we see in Lemma 2.26 below. This then by the above proposition, shall induce a surjective map between the Landstad algebras.

The injectivity part, to show that the Landstad algebras preserve exactness, requires a certain construction involving the kernel of the surjective map. So, given $\pi: B \rightarrow B^{\prime}$ a surjective morphism, satisfying the required conditions of Proposition 2.23 , consider the following $\hat{G}$-equivariant exact sequence

$$
0 \rightarrow \text { Ker } \pi \rightarrow B \rightarrow B^{\prime} \rightarrow 0
$$

Note, from the assumptions on the map $\pi$, the ideal Ker $\pi$ is invariant under the action $\hat{\rho}$. Hence, the dual action $\hat{\rho}$ descends to an action on the ideal Ker $\pi \subset B$ and shall still be denoted by $\hat{\rho}$. Then, we claim that Ker $\pi$ has a canonical $G$-product structure. The only extra work required to be a $G$-product, would be that of a compatible embedding of the group $G$ in $M(\operatorname{Ker} \pi)$. For that, define the following morphism $\beta: B \rightarrow M(\operatorname{Ker} \pi)$, associated with the ideal Ker $\pi \subset B$, as

$$
\beta(b) \cdot j=b \cdot j
$$

for all $b$ in $B$ and $j$ in Ker $\pi$. Since Ker $\pi$ is norm closed, by choosing an approximate unit that strictly converges to 1 in $M(\operatorname{Ker} \pi)$, we see that $\beta(b)=$ $b$, for all $b$ in Ker $\pi$. Then, the $*$-homomorphism $\beta$ is non-degenerate and hence we can extend it to $M(B)$. Now Ker $\pi$ being an ideal in $B$, which is an ideal in $M(B)$, one can show that Ker $\pi$ is also an ideal in $M(B)$, using an approximate unit for Ker $\pi$ in $B$. Hence the extended map shall be of the same form and we denote it also by $\beta$. Next, for all $g$ in $G$ and $\lambda_{g}$ in $M(B)$, we define $\tilde{\lambda}_{g}=\beta\left(\lambda_{g}\right) \in M(\operatorname{Ker} \pi)$. The map $g \mapsto \tilde{\lambda}_{g}$, a priori, need not be an embedding because the map $\beta$ is injective if and only if $\operatorname{Ker} \pi$ is essential in $B$. We include the following result that was not mentioned explicitly in [4].

Lemma 2.24. $\tilde{\lambda}_{g}=\tilde{\lambda}_{g^{\prime}}$ implies $g=g^{\prime}$.

Proof. The condition, $\tilde{\lambda}_{g}=\tilde{\lambda}_{g^{\prime}}$ implies $\lambda_{g} \cdot j=\lambda_{g^{\prime}} \cdot j$; for all $j$ in Ker $\pi$. Next, for any $\chi$ in $\hat{G}$, consider the dual action

$$
\begin{aligned}
\hat{\rho}_{\chi}\left(\lambda_{g} \cdot j\right) & =\hat{\rho}_{\chi}\left(\lambda_{g^{\prime}} \cdot j\right) ; \quad \forall j \in \operatorname{Ker} \pi \\
\hat{\rho}_{\chi}\left(\lambda_{g}\right) \hat{\rho}_{\chi}(j) & =\hat{\rho}_{\chi}\left(\lambda_{g^{\prime}}\right) \hat{\rho}_{\chi}(j) \\
\langle\chi, g\rangle \lambda_{g} \cdot \hat{\rho}_{\chi}(j) & =\left\langle\chi, g^{\prime}\right\rangle \lambda_{g^{\prime}} \cdot \hat{\rho}_{\chi}(j) ;
\end{aligned}
$$

But, $\lambda_{g} \cdot \hat{\rho}_{\chi}(j)=\lambda_{g^{\prime}} \cdot \hat{\rho}_{\chi}(j)$ from the hypothesis. Therefore, from the last equality above, we get

$$
\langle\chi, g\rangle=\left\langle\chi, g^{\prime}\right\rangle ; \quad \forall \chi \in \hat{G}
$$

Hence, $g=g^{\prime}$.

\footnotetext{
${ }^{16}$ This would mean $\mathrm{C}^{*}$-algebras with a strongly continuous action of the group $G$
} 
Then, the map $G \ni g \mapsto \tilde{\lambda}_{g} \in M(\operatorname{Ker} \pi)$ is a strictly continuous embedding of $G$ into $M(\operatorname{Ker} \pi)$. Moreover,

$$
\hat{\rho}_{\chi}\left(\tilde{\lambda}_{g}\right)=\langle\chi, g\rangle \tilde{\lambda}_{g}
$$

which then shows that the triple (Ker $\pi, \lambda, \hat{\rho})$ is a $G$-product.

Let $\mathcal{I}, A, A^{\prime}$ be Landstad algebras for the $G$-products $(\operatorname{Ker} \pi, \tilde{\lambda}, \hat{\rho}),(B, \lambda, \hat{\rho})$, $\left(B^{\prime}, \lambda^{\prime}, \hat{\rho}^{\prime}\right)$ respectively. Then, our next claim is that, the exact sequence 2.9 induces an exact sequence of the Landstad algebras.

$$
0 \rightarrow \mathcal{I} \rightarrow A \rightarrow A^{\prime} \rightarrow 0
$$

Let $\bar{\pi}$ be the morphism $\bar{\pi}: A \rightarrow A^{\prime}$ induced by $\pi: B \rightarrow B^{\prime}$. We had assumed that $\pi$ is surjective and hence by Proposition 2.23 , we have $\bar{\pi}(A)=A^{\prime}$. This then gives an exact sequence of the $\mathrm{C}^{*}$-algebras

$$
0 \rightarrow \operatorname{Ker} \bar{\pi} \rightarrow A \rightarrow A^{\prime} \rightarrow 0
$$

Also, we see that the morphism $\beta$ satisfies the assumptions of Proposition 2.23 and hence $\beta(A) \subset M(\mathcal{I})$. Thus, to arrive at the exact sequence 2.10, we just need to show that $\beta$ restricted to Ker $\bar{\pi}$ identifies it with the Landstad algebra $\mathcal{I}$. As showed in [4], one just need to check the following two conditions

1. $\beta(\operatorname{Ker} \bar{\pi})=\mathcal{I}$;

2. if $x \in \operatorname{Ker} \bar{\pi}$ and $\beta(x)=0$ then $x=0$.

Finally we summarize the above considerations in the following

Proposition 2.25. [4, Proposition 2.9] Let $(B, \lambda, \hat{\rho}),\left(B^{\prime}, \lambda^{\prime}, \hat{\rho}^{\prime}\right)$ be G-products with Landstad algebras $A, A^{\prime}$ respectively. Let $\pi$ be a nondegenerate surjective morphism from $B$ to $M\left(B^{\prime}\right)$, intertwining $\hat{\rho}$ and $\hat{\rho}^{\prime}$ such that $\pi\left(\lambda_{g}\right)=\lambda_{g}^{\prime}$. Then if $(\operatorname{Ker} \pi, \tilde{\lambda}, \hat{\rho})$ be the $G$-product as described above and $\mathcal{I} \subset M(\operatorname{Ker} \pi)$ its Landstad algebra. Then $\mathcal{I}$ can be embedded into $A$ and we have a $G$-equivariant exact sequence

$$
0 \rightarrow \mathcal{I} \rightarrow A \stackrel{\bar{\pi}}{\longrightarrow} A^{\prime} \rightarrow 0
$$

where $\bar{\pi}$ is the restriction of the nondegenerate surjective morphism $\pi: B \rightarrow$ $M\left(B^{\prime}\right)$ to $A$.

To see that Rieffel deformation preserves exactness, we prepare the first groundwork that equivariant surjective maps between $G$-C $\mathrm{C}^{*}$-algebras induce surjective maps between the crossed products.

Lemma 2.26. Given an equivariant surjective $*$-homomorphism $\phi: A \rightarrow A^{\prime}$ between two $G$ - $C^{*}$-algebras $A, A^{\prime}$ respectively, the induced $*$-homomorphism between the crossed product $C^{*}$-algebras is also surjective.

Proof. Sketch:

- Given the map $\phi: A \rightarrow A^{\prime}$, we can always induce a corresponding map $\tilde{\phi}: C_{c}(G ; A) \rightarrow C_{c}\left(G ; A^{\prime}\right)$ by composing the value of a function at an element $g$ in $G$ by the map $\phi$. 
- This map shall respect the covariance condition of the crossed products and hence extend to the completion of the algebra $C_{c}(G ; A)$ inside the crossed product $\mathrm{C}^{*}$-algebra $A \rtimes G$.

- $\phi$ is surjective, shall imply $\tilde{\phi}: C_{c}(G ; A) \rightarrow C_{c}\left(G ; A^{\prime}\right)$ is also surjective.

- But, the compactly supported continuous functions are dense in the crossed product algebra. Hence the image of the $*$-homomorphism $\tilde{\phi}$, and its extension to the completion $A \rtimes G$ is dense in the crossed product $A^{\prime} \rtimes G$.

- But the image of a $*$-homomorphism between $\mathrm{C}^{*}$-algebras is always closed. Hence the map induced by $\phi$ between the crossed products is surjective.

For our theory, we shall assume the requirement that the required unitization $\bar{A}$ comes with a group $G$-action $\rho$ that defines an action $\rho^{\prime}$ on the boundary quotient algebra $\bar{A} / A$. Given the group actions, we have the embedding $\lambda$ of the group $G$ into $M(\bar{A} \rtimes G)$ and that of $\lambda^{\prime}$ into $M((\bar{A} / A) \rtimes G)$ respectively. Finally as in Proposition 2.25 above, we construct the embedding $\lambda$ of $G$ into the kernel of the quotient map, which is $A$. Then consider the given $G$-equivariant extension $0 \rightarrow A \rightarrow \bar{A} \rightarrow \bar{A} / A \rightarrow 0$ as defining a noncommutative coarse structure on the $\mathrm{C}^{*}$-algebra $A$. By Lemma 2.26 , we consider the exact sequence of the corresponding crossed product algebras

$$
0 \rightarrow A \rtimes G \rightarrow \bar{A} \rtimes G \rightarrow(\bar{A} / A) \rtimes G \rightarrow 0
$$

In Kasprzak's approach to Rieffel deformation, using the isomorphism at the level of the crossed products, we can show that surjectivity of the map is preserved. By the crossed product isomorphisms, we have an exact sequence of the following deformed $G$-products $\left(A^{\Psi} \rtimes G, \tilde{\lambda}, \hat{\rho}^{\Psi}\right),\left(\bar{A}^{\Psi} \rtimes G, \lambda, \hat{\rho}^{\Psi}\right)$ and $\left((\bar{A} / A)^{\Psi} \rtimes G, \lambda^{\prime},\left(\hat{\rho}^{\prime}\right)^{\Psi}\right)$ respectively. Then by what we have shown in Proposition 2.25 above, their Landstad algebras shall form a $G$-equivariant exact sequence

$$
0 \rightarrow A^{\Psi} \rightarrow \bar{A}^{\Psi} \rightarrow(\bar{A} / A)^{\Psi} \rightarrow 0
$$

Finally we need to show that if $\bar{A}$ is unital then $\bar{A}^{\Psi}$ is unital. An algebra $\bar{A}$ is unital if and only if there is a nondegenerate $*$-homomorphism $\mathbb{C} \rightarrow \bar{A}$, that is equivariant for the trivial action of $G$ on $\mathbb{C}$. As above, Rieffel deformation maps this to a nondegenerate $*$-homomorphism $\mathbb{C}^{\Psi} \rightarrow \bar{A}^{\Psi}$. But for trivial action, as in Corollary 2.22, $\mathbb{C}^{\Psi}=\mathbb{C}$. Therefore $\bar{A}^{\Psi}$ is unital.

With this, starting with a noncommutative coarse structure on a $\mathrm{C}^{*}$-algebra $A$, we have thereby assigned a noncommutative coarse structure on the deformed algebra $A^{\Psi}$.

\subsubsection{Noncommutative coarse structure on the Moyal Plane}

As shown by Rieffel in 7], for any natural number $n$ the Moyal plane is Rieffel deformation of $C_{0}\left(\mathbb{R}^{2 n}\right)$ under the translation action of the vector group $\mathbb{R}^{2 n}$ on 
$C_{0}\left(\mathbb{R}^{2 n}\right)$ and the standard symplectic matrix $J$. Following the above procedure to assign noncommutative coarse structure on the deformed algebra by starting with one on the undeformed algebra, we shall assign a coarse structure on the Moyal Plane.

Given the metric coarse structure coming from the Euclidean metric on the classical plane $\mathbb{R}^{2 n}$, let us denote its Higson compactification by $h \mathbb{R}^{2 n}$. The Euclidean metric on $\mathbb{R}^{2 n}$ being a proper metric, by Theorem 1.13 in the previous chapter, we know that the metric coarse structure is the same as the topological coarse structure coming from $h \mathbb{R}^{2 n}$. Thus the following extension

$$
0 \rightarrow C_{0}\left(\mathbb{R}^{2 n}\right) \rightarrow C\left(h \mathbb{R}^{2 n}\right) \rightarrow C\left(\nu \mathbb{R}^{2 n}\right) \rightarrow 0
$$

defines a unique coarse structure on the classical plane $\mathbb{R}^{2 n}$. Here $\nu \mathbb{R}^{2 n}$ is the boundary of the Higson compactification and so $C\left(\nu \mathbb{R}^{2 n}\right) \cong C\left(h \mathbb{R}^{2 n}\right) / C_{0}\left(\mathbb{R}^{2 n}\right)$ Then using the procedure outlined in the previous section by Rieffel deformation, we define a noncommutative coarse structure on the Moyal plane. The following Lemma is a well-known fact, of which we give a proof below.

Lemma 2.27. The lift of the translation action of $\mathbb{R}^{2 n}$ on $\mathbb{R}^{2 n}$ to $C_{0}\left(\mathbb{R}^{2 n}\right)$ is strongly continuous.

Proof. For an element $f$ in $C_{0}\left(\mathbb{R}^{2 n}\right)$, consider the map $\alpha_{f}: \mathbb{R}^{2 n} \rightarrow C_{0}\left(\mathbb{R}^{2 n}\right)$ given by

$$
\alpha_{f}(t)(x)=f(x-t)
$$

Then given the euclidean metric on $\mathbb{R}^{2 n}$ and the supremum norm topology on $C_{0}\left(\mathbb{R}^{2 n}\right)$, we have to show that given a $t_{1} \in \mathbb{R}^{2 n}$; for every $\epsilon>0$ there exists $\delta>0$ such that for every $t_{2}$ with $\left\|t_{1}-t_{2}\right\|<\delta$ implies $\left\|\alpha_{f}\left(t_{1}\right)-\alpha_{f}\left(t_{2}\right)\right\|<\epsilon$. Now since $f \in C_{0}\left(\mathbb{R}^{2 n}\right)$, given $t_{1} \in \mathbb{R}^{2 n}$, there exists a compact set $K_{\epsilon}$ such that $\left|f\left(x-t_{1}\right)\right|<\epsilon / 3, \forall x \in\left\{\mathbb{R}^{2 n} \backslash K_{\epsilon}\right\}$. Now inside the compact set $K_{\epsilon}$, the function $\left|f\left(x-t_{1}\right)-f\left(y-t_{1}\right)\right|$ shall attain a maximum value and also the minimum value 0 when $x=y$. For each $x \in K_{\epsilon}$ consider the open ball $U_{x}$ such that for all $y \in U_{x} ;\left|f\left(x-t_{1}\right)-f\left(y-t_{1}\right)\right|<\epsilon$. Now, $K_{\epsilon}$ being a compact metric space, the open cover $\left\{U_{x}\right\}_{x}$ shall have a Lebesgue number, say $d_{f}^{t_{1}}$, depending only on the function $f, t_{1}$ and $\epsilon$ chosen.

Now for every $x, y \in\left\{\mathbb{R}^{2 n} \backslash K_{\epsilon}\right\},|f(x)-f(y)|<\epsilon / 3+\epsilon / 3<\epsilon$. Therefore the only points to worry about is those near the boundary of $K_{\epsilon}$. But for that, let $u_{f}^{t_{1}}$ be the lebesgue number for a covering of $K_{\epsilon}$ similar to the above one but now for the inequality $\left|f\left(x-t_{1}\right)-f\left(y-t_{1}\right)\right|<\epsilon / 3$. Then for any point $x$ in $\left\{\mathbb{R}^{2 n} \backslash K_{\epsilon}\right\}$, near to the boundary, such that the ball $B\left(x ; u_{f}^{t_{1}}\right)$ crosses into $K_{\epsilon}$ we can choose a point $x_{0}$ on the boundary of $K_{\epsilon}$ intersection with the ball $B\left(x ; u_{f}^{t_{1}}\right)$. Then for $y \in B\left(x ; u_{f}^{t_{1}}\right) \cap K_{\epsilon}$, we have $\left|f\left(x-t_{1}\right)-f\left(y-t_{1}\right)\right| \leq$ $\left|f\left(x-t_{1}\right)-f\left(x_{0}-t_{1}\right)\right|+\left|f\left(x_{0}-t_{1}\right)-f\left(y-t_{1}\right)\right| \leq\left|f\left(x-t_{1}\right)\right|+\left|f\left(x_{0}-t_{1}\right)\right|+$ $\left|f\left(x_{0}-t_{1}\right)-f\left(y-t_{1}\right)\right| \leq \epsilon$. This then gives that for all $x \in\left\{\mathbb{R}^{2 n} \backslash K_{\epsilon}\right\}$, and $y \in B\left(x ; u_{f}^{t_{1}}\right)$ we have $\left|f\left(x-t_{1}\right)-f\left(y-t_{1}\right)\right| \leq \epsilon$.

Then by choosing $\delta:=\min \left\{d_{f}^{t_{1}}, u_{f}^{t_{1}}\right\}$, for all $t_{2}$ such that $\left\|t_{1}-t_{2}\right\|<\delta$, we have $\left\|\alpha_{f}\left(t_{1}\right)-\alpha_{f}\left(t_{2}\right)\right\|<\epsilon$. Thus proving continuity of the map $\alpha_{f}$ at $t_{1} \in \mathbb{R}^{2 n}$. But $t_{1}$ chosen is arbitrary and hence the map $\alpha_{f}$ is continuous. 
Next we need to show that the translation action is strongly continuous on the Higson compactification. But before that we shall show that the translation action is trivial on the Higson corona. Let us first define the translation action of $\mathbb{R}^{2 n}$ on $C_{b}\left(\mathbb{R}^{2 n}\right)$ by

$$
\alpha_{t}(f)(x)=f(x-t)
$$

where $x, t$ are elements of $\mathbb{R}^{2 n}$. Then we show the following

Lemma 2.28. Let $f$ in $C_{b}\left(\mathbb{R}^{2 n}\right)$ be a Higson function for the metric coarse structure on $\mathbb{R}^{2 n}$, then $\alpha_{t}(f)-f$ is in $C_{0}\left(\mathbb{R}^{2 n}\right)$ for all $t \in \mathbb{R}^{2 n}$.

Proof. Clearly, if $f \in C\left(h \mathbb{R}^{2 n}\right)$ then for any $t \in \mathbb{R}^{2 n}$, consider the controlled set $E_{t}:=\{(x, y):\|x-y\| \leq\|t\|\}$. Then $f$ being a Higson function, we have that $d f \in C_{0}\left(E_{t}\right)$. Therefore $\left|\left(\alpha_{t}(f)-f\right)(x)\right|=|f(x-t)-f(x)| \rightarrow 0$ as $x \rightarrow \infty$.

This shows that the translation action $\alpha$ descends to the trivial action on the Higson corona, the quotient $\mathrm{C}^{*}$-algebra $C\left(h \mathbb{R}^{2 n}\right) / C_{0}\left(\mathbb{R}^{2 n}\right) \cong C\left(\nu \mathbb{R}^{2 n}\right)$. Being the trivial action, the map $\alpha_{[f]}: t \mapsto \alpha_{t}([f])$ is the constant map and hence continuous for all $[f] \in C\left(\nu \mathbb{R}^{2 n}\right)$. This then gives that the action on the quotient $\mathrm{C}^{*}$-algebra is strongly continuous. Finally, the main result in [3] says that if a locally compact group acts isometrically on a Banach space $X$ leaving a closed subspace $M$ invariant, and if the induced actions on $M$ and $X / M$ are strongly continuous, then the action on $X$ is strongly continuous. With this we have that the translation action is strongly continuous on the Higson compactification.

In fact, one can also prove a stronger result in the converse direction. This shall prove to be important as to why we do not expect coarse equivalence of the metric coarse structure on $\mathbb{R}^{2 n}$ with other noncommutative coarse structures on the Moyal plane, coming from Rieffel deformation of other compactifications of $\mathbb{R}^{2 n}$.

Lemma 2.29. For $f \in C_{b}\left(\mathbb{R}^{2 n}\right)$ and the translation action $\alpha, \alpha_{t}(f)-f$ belongs to $C_{0}\left(\mathbb{R}^{2 n}\right)$ for all $t \in \mathbb{R}^{2 n}$. Then $f \in C\left(h \mathbb{R}^{2 n}\right)$.

Proof. Given that $\alpha_{t}(f)-f$ belongs to $C_{0}\left(\mathbb{R}^{2 n}\right)$, we have that given $\epsilon>0$, there exists a bounded radius of a compact set, say $R>0$, such that for all $x \in \mathbb{R}^{2 n}$ and $\|x\|>R$ we have that $\|f(x-t)-f(x)\|<\epsilon$. Further as we noted above, from 3 we know that the map $\alpha_{f}: \mathbb{R}^{2 n} \rightarrow\left(C_{0}\left(\mathbb{R}^{2 n}\right),\|\|_{\text {sup }}\right)$ given by $t \mapsto \alpha_{t}(f)-f$ is continuous on $\mathbb{R}^{2 n}$. Therefore, by Arzela-Ascoli's theorem, the map shall be uniformly continuous on a compact subset, say $[-1,1]^{2 n} \subset \mathbb{R}^{2 n}$. Next, using this two conditions, we prove the Higson condition for the function $f$.

Now $f \in C\left(h \mathbb{R}^{2 n}\right)$, if for any $x, y \in \mathbb{R}^{2 n}$ within any bounded set $E_{N}:=\{(x, y)$ : $\|x-y\|<N\}$ for $N>0$, we have $|f(x)-f(y)| \rightarrow 0$ as $\|x\| \rightarrow \infty$. Then fixing $N>0$, consider any $x, y$ such that $\|x-y\|<N$. Now given that the map $\alpha_{f}$ is uniformly continuous on the compact set $[-1,1]^{2 n}$, we can divide any path between $x$ and $y$ by $N-1$ points such that the distance between any two successive points is less than 1 . With this, consider the inequality

$$
|f(x)-f(y)| \leq \Sigma_{k}\left|f\left(x+\frac{y-x}{N} k\right)-f\left(x+\frac{y-x}{N}(k+1)\right)\right| ; \quad k=0, \ldots N
$$


Then given $\epsilon>0$, we can choose $R>0$ such that for $\|x\|>R+N$, and all $k=0, \ldots N-1$ we have

$$
\begin{aligned}
& \left|f\left(x+\frac{y-x}{N} k\right)-f\left(x+\frac{y-x}{N}(k+1)\right)\right| \\
& =\left|\alpha_{\frac{y-x}{N}} f\left(x+\frac{y-x}{N}(k+1)\right)-f\left(x+\frac{y-x}{N}(k+1)\right)\right| \leq \epsilon
\end{aligned}
$$

Then we have that $|f(x)-f(y)| \leq N \epsilon$ for any $(x, y) \in E_{N}$ and $\|x\| \geq R+N$. Since $N$ chosen is arbitrary, this proves that $f$ is a Higson function.

This then gives us that the translation action gives an $\mathbb{R}^{2 n}$-equivariant exact sequence

$$
0 \rightarrow C_{0}\left(\mathbb{R}^{2 n}\right) \rightarrow C\left(h \mathbb{R}^{2 n}\right) \rightarrow C\left(\nu \mathbb{R}^{2 n}\right) \rightarrow 0
$$

We then apply Lemma 2.26 to arrive at an exact sequence of the crossed product algebras

$$
0 \rightarrow C_{0}\left(\mathbb{R}^{2 n}\right) \rtimes_{\alpha} \mathbb{R}^{2 n} \rightarrow C\left(h \mathbb{R}^{2 n}\right) \rtimes_{\alpha} \mathbb{R}^{2 n} \rightarrow C\left(\nu \mathbb{R}^{2 n}\right) \rtimes_{\alpha} \mathbb{R}^{2 n} \rightarrow 0
$$

Finally for the standard symplectic matrix $J$ on $\mathbb{R}^{2 n}$ and the corresponding 2cocycle $\Psi_{J}(x, y):=e(x \cdot J y)$, we use the isomorphism as in Proposition 2.16 to arrive at the deformed $\mathbb{R}^{2 n}$-products of the above crossed products. Note that the deformation procedure do not spoil the exactness property of the above extension. With this, we arrive at the following exact sequence of crossed products for the deformed algebras.

$$
\begin{array}{r}
0 \rightarrow C_{0}\left(\mathbb{R}^{2 n}\right)^{\Psi_{J}} \rtimes_{\alpha^{\Psi_{J}}} \mathbb{R}^{2 n} \rightarrow C\left(h \mathbb{R}^{2 n}\right)^{\Psi_{J}} \rtimes_{\alpha^{\Psi_{J}}} \mathbb{R}^{2 n} \rightarrow \\
C\left(\nu \mathbb{R}^{2 n}\right)^{\Psi_{J}} \rtimes_{\alpha^{\Psi_{J}}} \mathbb{R}^{2 n} \rightarrow 0
\end{array}
$$

Finally, we apply Proposition 2.25 to the above exact sequence, to arrive at a noncommutative coarse structure on the Moyal plane $C_{0}\left(\mathbb{R}^{2 n}\right)^{\Psi_{J}}$.

$$
0 \rightarrow C_{0}\left(\mathbb{R}^{2 n}\right)^{\Psi_{J}} \rightarrow C\left(h \mathbb{R}^{2 n}\right)^{\Psi_{J}} \rightarrow C\left(\nu \mathbb{R}^{2 n}\right)^{\Psi_{J}} \rightarrow 0
$$

Remark 2.30. To define a noncommutative coarse structure on the Moyal plane, we can also choose some other compactification of $\mathbb{R}^{2 n}$ as long as the translation action is strongly continuous on the boundary quotient algebra. But observe that by Lemma 2.29 the translation action of $\mathbb{R}^{2 n}$ on $C_{b}\left(\mathbb{R}^{2 n}\right)$ is trivial only on the boundary quotient algebra of the Higson compactification given by the metric coarse structure on $\mathbb{R}^{2 n}$. As we have noted before, in the section on Rieffel deformation under trivial action, the boundary quotient algebra of the Moyal plane shall then be the same as the original Higson corona $C\left(\nu \mathbb{R}^{2 n}\right)$. In the next chapter we shall see that this $*$-isomorphism between the boundary quotient algebra is crucial to ensure coarse equivalence of noncommutative coarse structures. Therefore we do not expect a coarse equivalence between the Moyal plane and other topological coarse structures on the classical plane. 


\section{Chapter 3}

\section{Noncommutative coarse maps}

We begin with the definition of noncommutative coarse maps between non-unital $\mathrm{C}^{*}$-algebras, with coarse structure coming from a unitization. In Chapter 1 such a noncommutative coarse structure has been defined as an extension of $\mathrm{C}^{*}$-algebras. We show that the boundary quotient algebra plays a major role in the formulation. Thus the most prominent examples of our theory, shall be those with commutative boundary quotients. This allows us to compare with the classical theory of topological coarse structures. In this chapter, we shall study the plausible morphisms between such noncommutative coarse structures.

\subsection{Noncommutative coarse maps}

We begin with the definition of the main object of this chapter.

Definition 3.1. Let $A, B$ be non-unital $\mathrm{C}^{*}$-algebras with noncommutative coarse structures given by certain extensions

$$
0 \rightarrow A \rightarrow \bar{A} \rightarrow \bar{A} / A \rightarrow 0 ; \quad 0 \rightarrow B \rightarrow \bar{B} \rightarrow \bar{B} / B \rightarrow 0
$$

respectively. We define a strictly continuous, contractive, completely positive map $\phi: A \rightarrow B$ to be a noncommutative coarse map if the following diagram is satisfied along with the conditions and properties mentioned in the next paragraph.

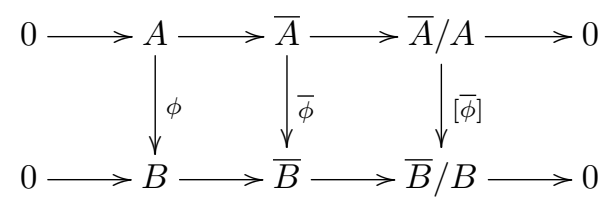

The requirements on the maps are: 
1. Since $A$ is dense in the strict topology of $M(A)$, the strictly continuous map $\phi$ uniquely extends to the multiplier algebras $\bar{\phi}: M(A) \rightarrow M(B)$.

2. We require $\bar{\phi}$ to be a unital map because we want pure states to be mapped into states or probability measures in the commutative case. As we shall see in the commutative case this consideration comes from the use of a certain partition of unity with which we construct completely positive coarse maps from a given classical coarse map.

3. The important point for the map $\phi$ to be coarse with respect to the given unitizations, should then be that the image of the restriction of $\bar{\phi}$ to the unitization $\bar{A}$ should be contained in $\bar{B}$. We should have that $\left.\bar{\phi}\right|_{\bar{A}}(\bar{A}) \subset \bar{B}$.

4. Finally, the map $\bar{\phi}$ should descend to a $*$-homomorphism with respect to the boundary quotient algebras of the given unitizations. Namely, $[\bar{\phi}]: \bar{A} / A \rightarrow \bar{B} / B$ should be a $*$-homomorphism.

Remark 3.2. The extension $\bar{\phi}$ being a unital map, shall automatically require the map $\phi$ to be a contractive completely positive map. One can show that for an approximate unit $\left\{u_{\alpha}\right\}_{\alpha}$, the net $\left\{\left\|\phi\left(u_{\alpha}\right)\right\|\right\}_{\alpha}$ shall converge to $\|\phi\|$ the norm of the completely positive map. Then we know from the extension $\bar{\phi}$ being unital that $\|\phi\|$ should be 1 .

\subsubsection{Hilbert $C^{*}$-modules and completely positive maps}

Even though for our present work, we could completely skip the treatment of Hilbert $\mathrm{C}^{*}$-modules, we believe that future developments of our work shall rely more on using this path. Thus we give a short introduction to Hilbert $\mathrm{C}^{*}$-modules, to keep the broader setting in the backdrop. Then we look at completely positive maps between $\mathrm{C}^{*}$-algebras, which forms the class of our noncommutative coarse maps.

Definition 3.3. Given $A$ is a $\mathrm{C}^{*}$-algebra, an inner-product $A$-module(or pre-Hilbert $A$-module $)$ is a linear space $E$ which is a right $A$-module, with compatible scalar multiplication, $\lambda(x a)=(\lambda x) a=x(\lambda a)$ for all $x \in E, \lambda \in \mathbb{C}$ and $a \in A$, together with a map (an $A$-valued inner-product) $\langle\rangle:, E \times E \rightarrow A$, satisfying the following properties for all $x, y, z \in E$ and $\alpha, \beta \in \mathbb{C}$

1. $\langle x, \alpha y+\beta z\rangle=\alpha\langle x, y\rangle+\beta\langle x, z\rangle$;

2. $\langle x, y \alpha\rangle=\langle x, y\rangle \alpha$

3. $\langle y, x\rangle=\langle x, y\rangle^{*}$;

4. $\langle x, x\rangle \geqslant 0$; and if $\langle x, x\rangle=0$ then $x=0$.

Remark 3.4. Note the convention used, namely that condition (1) above requires the inner product to be linear in the second variable while condition (3) shall require it to be conjugate linear in the first variable. With this, we hereafter adopt the same condition for the ordinary inner product spaces and Hilbert spaces over $\mathbb{C}$. Therefore any inner product on the Hilbert space $(H,\langle\rangle$,$) shall$ be conjugate linear in the first variable and only linear in the second variable. 
For $x$ in the inner-product $A$-module $E$, define $\|x\|_{E}:=\|\langle x, x\rangle\|_{A}^{1 / 2}$. It can be shown that $\|\langle x, y\rangle\| \leq\|x\|\|y\|$ from which one can deduce that if $E$ is an inner-product $A$-module, then $\|\cdot\|_{E}$ is a norm on $E$. With this an inner-product $A$-module $E$ over the $\mathrm{C}^{*}$-algebra $A$ that is complete with respect to the norm $\|.\|_{E}$ shall be called a Hilbert $A$-module.

Example 3.5. For a given $\mathrm{C}^{*}$-algebra $A$, consider the linear space $A$ with the $A$-valued inner product

$$
\langle a, b\rangle:=a^{*} b
$$

where $a^{*}$ is the adjoint of $a$ in $A$. Clearly from the condition $\left\|a^{*} a\right\|=\|a\|^{2}$ of a $\mathrm{C}^{*}$-norm, we have that the norm on $A$ as an inner-product $A$-module as defined above coincides with the $\mathrm{C}^{*}$-algebra norm on $A$. Thus $A$ is complete as an innerproduct $A$-module and is therefore a Hilbert $A$-module. In the following theory, for a given $\mathrm{C}^{*}$-algebra $A$ we shall only be interested in the Hilbert $A$-module $A$ itself, as in this example.

Corresponding to Hilbert $A$-modules $E, F$ respectively, we define the set of adjointable operators $\mathcal{L}(E, F)$ as the set of linear $A$-module maps $t: E \rightarrow F$ such that there exists $t^{*}: F \rightarrow E$ satisfying the condition

$$
\langle t x, y\rangle=\left\langle x, t^{*} y\right\rangle ; \quad \forall x \in E, y \in F
$$

Using Banach-Steinhaus theorem, it can be shown that such a map $t$ is bounded and we call $\mathcal{L}(E, F)$ the set of adjointable maps from $E$ to $F$. In particular $\mathcal{L}(E)$ shall mean the set of all adjointable maps from $E$ to itself. This is an associative $*$-algebra with the involution $*$ given by the adjoint operator and the multiplication is defined as composition of operators. For the Hilbert $A$ module $E=A$, the algebra of adjointable operators $\mathcal{L}(A)$ is the multiplier algebra $M(A)$ of $A$.

There is the obvious operator norm for the elements of $\mathcal{L}(E)$, with respect to which it becomes a $\mathrm{C}^{*}$-algebra. But for the conditions on a noncommutative coarse map, we shall mainly be interested in the strict topology on the $\mathrm{C}^{*}$-algebra of adjointable operators $\mathcal{L}(E)$. It is defined as the topology generated by the following seminorms

$$
t \mapsto\|t x\| ; \quad \forall x \in E \quad t \mapsto\left\|t^{*} y\right\| ; \quad \forall y \in E
$$

It is a trivial observation that for the Hilbert $A$-module $A$, the strict topology on the algebra of adjointable operators $\mathcal{L}(A)=M(A)$, coincides with the strict topology on the multiplier algebra $M(A)$.

Having developed a short introduction to Hilbert $\mathrm{C}^{*}$-modules, let us next consider the notion of completely positive maps, that we have used to define a noncommutative coarse map. Considering $\mathrm{C}^{*}$-algebras as Banach spaces in the first place, we automatically have the notion of linear maps between them. A completely positive map is a special kind of positive linear map between $\mathrm{C}^{*}$-algebras, that preserve positive elements even at the level of the matrix algebras.

\footnotetext{
${ }^{1}$ A linear map $\sigma: A \rightarrow B$ is positive, if for every positive element $a^{*} a$ in $A, \sigma\left(a^{*} a\right)$ is positive in $B$.
} 
Given a linear map $\sigma: A \rightarrow B$ between $\mathrm{C}^{*}$-algebras and $n$ a positive integer, we define $\rho^{(n)}$ for the map from $M_{n}(A)$ to $M_{n}(B)$ obtained by applying $\rho$ to each matrix element

$$
\rho^{(n)}\left(\left(a_{i j}\right)\right):=\left(\rho\left(a_{i j}\right)\right)
$$

Definition 3.6. We say that $\rho$ is completely positive if all the maps $\rho^{(n)}, n \geq 1$ are positive.

Remark 3.7. Note that, a $*$-homomorphism between $\mathrm{C}^{*}$-algebras is always a completely positive map. In our definition of a noncommutative coarse map above, the completely positive map between the unitizations $\bar{\phi}: \bar{A} \rightarrow \bar{B}$ shall surely induce a completely positive map $[\bar{\phi}]$ between the boundary quotient algebras. For the map to be coarse, we require that $[\bar{\phi}]$ should actually be a $*$-homomorphism. This is motivated by the fact that a coarse map between topological coarse structures in the commutative case, induces a continuous map between the boundaries, as we shall see later.

In the commutative case, let $f: X \rightarrow Y$ be a continuous proper map between the topological spaces $X, Y$ respectively. A proper map pulls back compact sets in $Y$ to relatively compact sets in $X$. This condition guarantees the image of the induced map $f^{*}: C_{0}(Y) \rightarrow C(X)$, to be contained in $C_{0}(X)$. Further, $f^{*}$ shall then map any approximate unit in $C_{0}(Y)$ to an approximate unit in $C_{0}(X)$. This is a necessary condition for the $*$-homomorphism $f^{*}: C_{0}(Y) \rightarrow C_{0}(X)$ to extend to a unital map between the unitizations. The $*$-homomorphism $f^{*}$ is then said to be non-degenerate.

Definition 3.8. An approximate unit for a $\mathrm{C}^{*}$-algebra $A$ is a net $\left\{u_{\alpha}\right\}_{\alpha}$ of positive elements in $A$, each of norm less than or equal to one, such that

(a) $u_{\alpha} \geqslant u_{\beta}$ if $\alpha \geqslant \beta$, and

(b) for every $a \in A, \lim _{\alpha \rightarrow \infty}\left\|a u_{\alpha}-a\right\|=0$.

Example 3.9. The approximate units have an explicit construction in the commutative case. If $A=C_{0}(X)$ (for example $X$ is a second countable locally compact space) then by starting with open sets, $U_{1}, U_{2}, \ldots$, such that

(a) $\cup_{n} U_{n}=X$, and

(b) $\overline{U_{n}}$ is compact and contained in $U_{n+1}$.

Since $X$ is normal, by Urysohn's lemma, take $u_{n}$ to be a continuous, $[0,1]$-valued function which is supported in $U_{n+1}$ and identically 1 on $U_{n}$. Then clearly $\left\{u_{n}\right\}_{n}$ shall be an approximate unit for $C_{0}(X)$.

If $\left\{U_{n}\right\}_{n} ; U_{n} \subseteq Y$, corresponds to an approximate unit in $C_{0}(Y)$, then one can form a collection of compact sets $K_{n}$ containing $U_{n}$ like in Example 3.9 With this, the map $f: X \rightarrow Y$ being proper, it pulls back the compact sets $K_{n}$ to relatively compact sets $f^{-1}\left(K_{n}\right)$, as in the definition above. Then the pullbacks $f^{-1}\left(U_{n}\right)$ being contained in $f^{-1}\left(K_{n}\right)$ and having similar convergence criterion, 
shall also correspond to an approximate unit for $X$ as in Example 3.9 This is how the composition map $f^{*}: C_{0}(Y) \rightarrow C_{0}(X)$ shall map an approximate unit in $C_{0}(Y)$ to an approximate unit in $C_{0}(X)$.

In fact given a $\mathrm{C}^{*}$-algebra, one can explicitly write down an approximate unit by using the partially ordered set of positive elements with norm less than or equal to 1 . The details can be looked up in the following proposition

Theorem 3.10 ([6, Theorem 1.4.2]). Every $C^{*}$-algebra contains an approximate unit.

Remark 3.11. If $A$ is separable, the approximate unit can be chosen to be a sequence $\left\{u_{n}\right\}_{n \in \mathbb{N}}$.

For a completely positive map $\rho: A \rightarrow B$ between non-unital $\mathrm{C}^{*}$-algebras, let us first consider its nondegeneracy properties, as to what it would mean to have an extension to the multiplier algebras. Since $B \subset M(B)$, we can easily look at the map $\rho$ as $\rho: A \rightarrow M(B)$, or equivalently as $\rho: A \rightarrow \mathcal{L}(B)$. Thus, essentially we are reduced to consider nondegeneracy properties of completely positive maps $\rho: A \rightarrow \mathcal{L}(E)$ from a $\mathrm{C}^{*}$-algebra to the adjointable operators on some Hilbert $B$-module.

The additional condition that the Cauchy net $\left\{\rho\left(u_{\alpha}\right)\right\}_{\alpha}$ actually converges to 1 in the strict topology, is equivalent to that $\rho$ maps an approximate unit of $A$ to an approximate unit of $B$.

Definition 3.12. A completely positive map $\rho: A \rightarrow \mathcal{L}(E)$ is called nondegenerate, if $\rho\left(u_{\alpha}\right)$ strictly converges to 1 in $\mathcal{L}(E)$ for some approximate unit $\left\{u_{\alpha}\right\}_{\alpha}$ in $A$.

Therefore we see the notion of $\rho: A \rightarrow B$ being strictly continuous along with the extension $\bar{\rho}: M(A) \rightarrow M(B)$ being unital is equivalent to $\rho$ being nondegenerate. But in our definition, we have separated the two notions precisely to show the difference between the requirements as to why a noncommutative coarse map should be strictly continuous and on the other hand extend to a unital map between the multiplier algebras. In fact such an extension is uniquely defined as $\bar{\rho}(a)=\lim _{\alpha} \rho\left(a u_{\alpha}\right)$; for some approximate unit $u_{\alpha}$ in $A$.

With this our definition of a noncommutative coarse map is complete. Now we would like to motivate this definition from classical coarse maps. For that we next look at the theory of coarse maps between classical coarse spaces. We mainly consider proper coarse structures on a paracompact, locally compact and Hausdorff topological space, the one of interest for generalizing to the noncommutative setting.

\subsection{Coarse maps}

The speciality about coarse geometry is to give more priority to the large scale structure of the space rather than on the local nature around a point. Continuity of maps between topological spaces require that the local neighbourhoods around a point are preserved, when pulled back by the map. This is no more 
required from the perspective of coarse geometry. Hence, most often, continuity condition is not assumed for maps relating coarse structures between two coarse spaces.

This, as we saw above, requires us to broaden from $*$-homomorphisms between $\mathrm{C}^{*}$-algebras in the algebraic formulation of a coarse map. Luckily, the boundary of the compactification defining a topological coarse structure serves as a functor to the category of compact topological spaces with continuous maps. Given a discontinuous coarse map, we show we can construct a completely positive map between the $\mathrm{C}^{*}$-algebra of the compactifications that restrict to the same boundary *-homomorphism as the honest coarse map. This is how we plan to motivate in this section our definition of a noncommutative coarse map by considering the algebraic implications of a coarse map between coarse structures given by compactifications in the commutative case.

Definition 3.13. A map $f: X \rightarrow Y$ between coarse spaces is called proper if the pullback of every bounded subset in $Y$ is a bounded subset of $X$.

Recall that we are mainly interested in a proper coarse space, in which every bounded subset is required to be relatively compact. Thus, a map $f: X \rightarrow Y$ between coarse spaces is proper would imply that it is proper in the sense of a map between topological spaces, pulling back compact sets in $Y$ to relatively compact sets in $X$. The properness condition on a map $f: X \rightarrow Y$, conveys the idea that points going out to the boundary in $X$ are mapped to points going out to the boundary in $Y$. The identity map id: $X \rightarrow X$ for any topological space $X$ is always a proper map. Whereas the constant map $f: X \rightarrow Y$ that maps the whole of a non-compact space $X$ to a constant point $y_{0}$ in $Y$ is not proper.

Definition 3.14. A map between coarse spaces $f: X \rightarrow Y$ is called bornologous if it maps controlled sets to controlled sets.

Example 3.15. For the space of integers $\mathbb{Z}$ with the metric coarse structure coming from the metric $d(x, y)=|x-y|$, the map translation by a finite amount $n \mapsto n+k$ for some fixed $k>0$ is bornologous. But the map $n \mapsto n^{2}$ is not.

In coarse geometry we shall be interested in maps, not necessarily continuous, but that satisfy both of these conditions.

Definition 3.16. A map between coarse spaces is a coarse map if it is both proper and bornologous.

There is a very important equivalence relation called closeness on the set of all coarse maps between two coarse spaces. In coarse geometry it is enough to define maps up to this equivalence relation.

Definition 3.17. Two coarse maps $f, g: X \rightarrow Y$ between coarse spaces $X, Y$ are called close if the set $\{(f(x), g(x)): \forall x \in X\}$ is a controlled set in $Y$.

Given the equivalence relation of closeness between two coarse maps, we next consider the notion of coarse equivalence between two coarse spaces. 
Definition 3.18. A coarse map $f: X \rightarrow Y$ is a coarse equivalence, if there exists another coarse map $g: Y \rightarrow X$ such that $f \circ g$ is close to $\operatorname{id}_{Y}$ and $g \circ f$ is close to id ${ }_{X}$.

For example the inclusion map $i: \mathbb{Z} \rightarrow \mathbb{R}$ is a coarse equivalence, with the inverse coarse map being given by mapping $r \in \mathbb{R}$ to the largest integer less than it. We shall be more interested in noncommutative coarse equivalence and shall come back to it later in section 3.4 of this chapter.

\subsubsection{Continuous coarse maps for topological coarse struc- tures}

In this section, we shall briefly look at the known results concerning continuous coarse maps between topological coarse structures. Let us start with the following theorem from [9].

Theorem 3.19 (9, Proposition 2.39]). Let $X$ and $Y$ be locally compact Hausdorff spaces provided with second countable compactifications $\bar{X}$ and $\bar{Y}$ respectively. Then a continuous and proper map $f: X \rightarrow Y$ is coarse with respect to the topological coarse structure coming from the compactifications iff it extends to a continuous map $\bar{f}: \bar{X} \rightarrow \bar{Y}$.

It is illuminating to see that even for a nice map like the identity map on a topological space, it might fail to be coarse with respect to coarse structure coming from two different compactifications, when the map do not extend to a continuous map on the boundary.

Example 3.20. Consider two different compactifications on the Real line $\mathbb{R}$, namely, the one-point compactification corresponding to the circle $S^{1}$ and the two end point compactification that is homeomorphic to $[0,1]$. The identity map id: $\mathbb{R} \rightarrow \mathbb{R}$ is obviously proper and continuous but it does not have any continuous extension to any map id: $S^{1} \rightarrow[0,1]$. This can be seen from the fact that the point at infinity, say $x_{\infty} \in S^{1}$ is mapped to 0 w.l.o.g., then the pullback of the open neighbourhoods of 0 in $[0,1]$ is not open in $S^{1}$. Also we see that the map id: $\mathbb{R} \rightarrow \mathbb{R}$ is not bornologous. Consider the net $\{(\lambda,-\lambda)\}_{\lambda>0}$ it is controlled in the topological coarse structure on $\mathbb{R}$ coming from $S^{1}$ but its image under the identity map is not controlled in the coarse structure coming from the two-point compactification $[0,1]$.

Thus from Theorem 3.19, for coarse structures coming from a second countable compactification a continuous coarse map $f: X \rightarrow Y$ shall extend to a continuous map $\bar{f}: \bar{X} \rightarrow \bar{Y}$. Finally, we then have the following diagram as defining an algebraic picture of a coarse map between $\mathrm{C}^{*}$-algebras.

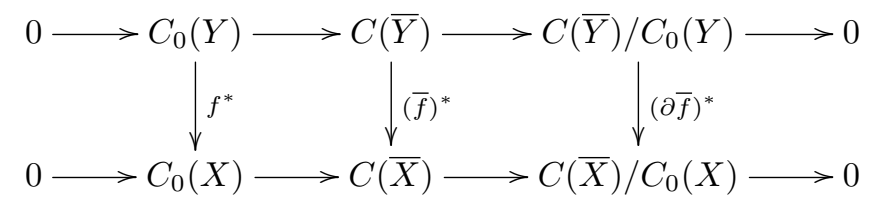


Here $f^{*} \sqrt{2}(\bar{f})^{*}$ are the maps induced between the algebras of continuous functions by composition with $f, \bar{f}$ respectively and $(\partial \bar{f})^{*}$ is the restriction of $(\bar{f})^{*}$ to the boundary quotient algebras. Continuity of $f$ implies that all of these maps are $*$-homomorphisms and hence it satisfies our definition of a noncommutative coarse map.

\subsection{Discontinuous coarse maps}

Having looked at the set-up for the corresponding algebraic diagram induced by a continuous coarse map, in this section we shall look at the situation for discontinuous coarse maps. In particular we shall be interested in motivating our definition for noncommutative coarse maps.

Since we replace coarse structures by compactifications, it is important to see when do the compactification uniquely determines the coarse structure. Starting with a compactification and its proper topological coarse structure we can define its Higson compactification and then compare with the original compactification. We refer to the following work of Roe which shows that for a compactification to uniquely define a proper coarse structure it needs to be second countable.

Proposition 3.21. 9, Proposition 2.48] Suppose that $X$ is a locally compact Hausdorff space, and that it is given a topological coarse structure associated to a second countable compactification $\bar{X}$. Then the Higson compactification of $X$ is the originally given compactification.

It is interesting to note that in the proof above, an important criteria is to construct a sequence in $X$ that should converge to a certain given boundary point $x_{0} \in \partial \bar{X}$ without having any other accumulation points at the boundary. While second countable compactifications guarantee that as in [9], we can also consider a more general topological framework. In the next lemma we show that given $X$ is $\sigma$-compact and the boundary $\partial \bar{X}$ is second countable, we can construct a sequence $\left\{x_{n}\right\}_{n}$ that converges to a given point $x_{0}$ in $\partial \bar{X}$ and has no other accumulation point on the boundary.

Lemma 3.22. Let $X$ be $\sigma$-compact with a compactification $\bar{X}$ such that the boundary $\partial \bar{X}$ is metrizable. Then there exists a sequence $\left\{x_{n}\right\}_{n}$ that converges to the given boundary point $x_{\infty}$ and has no other accumulation points on the boundary.

Proof. To see this, we first consider a metrizable quotient space $\overline{X^{\prime}}$ of $\bar{X}$. Start with a sequential approximate unit $\left\{u_{m}\right\}_{m}$ of $C_{0}(X)$. Now for every $f$ in $C(\partial \bar{X})$, consider the extension $\bar{f}$ of $f$ to $C(\bar{X})$. This exists because $\partial \bar{X}$ is a closed subset of $\bar{X}$ which being a compact Hausdorff space is normal. But $C(\partial \bar{X})$ is separable. Consider $\left\{\overline{f_{n}}\right\}_{n}$ the collection of extensions of a countable dense subset $\left\{f_{n}\right\}_{n}$ of $C(\partial \bar{X})$. Then the $\mathrm{C}^{*}$-algebra $C\left(\overline{X^{\prime}}\right)$ generated by $\left\{1, u_{m}, \overline{f_{n}}: n, m \in \mathbb{Z}\right\}$ is a separable $\mathrm{C}^{*}$-subalgebra of $C(\bar{X})$. We then have the following diagram of

\footnotetext{
${ }^{2}$ Note that for the image of the map $f^{*}$ to be inside $C_{0}(X)$, the map $f$ should be proper at the first place.
} 
extensions

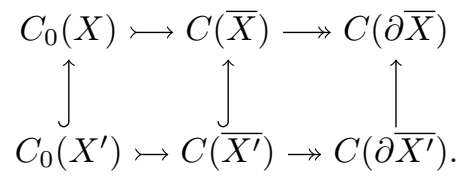

The inclusion map $C_{0}\left(X^{\prime}\right) \hookrightarrow C_{0}(X)$ is non-degenerate because its image contains an approximate unit of $C_{0}(X)$. Since the extension in the top row is essential, the same holds for the bottom row of the above diagram. This gives that $X^{\prime} \subseteq \overline{X^{\prime}}$ is dense and thus $\overline{X^{\prime}}$ is a compactification of $X^{\prime}$. An important point of this construction is that the boundaries $\partial \bar{X} \cong \partial \overline{X^{\prime}}$ are homeomorphic.

Since $C\left(\overline{X^{\prime}}\right)$ is separable, $\overline{X^{\prime}}$ is a metric space. Consider the metric $d_{\overline{X^{\prime}}}$ on $\overline{X^{\prime}}$. The inclusion map $C\left(\overline{X^{\prime}}\right) \hookrightarrow C(\bar{X})$ is dual to a continuous map $r: \bar{X} \rightarrow \overline{X^{\prime}}$. We define $d_{\bar{X}}:=d_{\overline{X^{\prime}}} \circ(r \times r)$. This is then a quasi-metric on $\bar{X}$. But since the boundaries are homeomorphic, that is, $\left.r\right|_{\partial \bar{X}}$ is the identity map, $d_{\bar{X}}$ restricts to the original metric on the boundary $\partial \bar{X}$ and defines its topology.

Now, let $\left\{U_{n}\right\}_{n}$ be the collection of compact sets corresponding to the approximate unit $\left\{u_{n}\right\}_{n}$ in $C_{0}(X)$. Given $x_{\infty} \in \partial \bar{X}$ we consider the closed balls $\left\{B\left(x_{\infty} ; 2^{-n}\right):=\left\{x \in \bar{X}: d_{\bar{X}}\left(x, x_{\infty}\right) \leq 2^{-n}\right\}_{n}\right.$ in $\bar{X}$. For a given $n$, we choose a point $x_{n}$ in $X$ such that it satisfies the following conditions

$$
x_{n} \notin U_{n}, \quad x_{n} \in B\left(x_{\infty} ; 2^{-n}\right) .
$$

Then as $n$ tends to $\infty$, the points $x_{n}$ are pushed to the boundary by the first condition. Also since on the boundary the function $d_{\bar{X}}$ defines its topology, with $n \rightarrow \infty$ the radius $2^{-n}$ of the balls $B\left(x_{\infty} ; 2^{-n}\right)$ shrinks to 0 and so there are no other possible accumulation points of the sequence $\left\{x_{n}\right\}_{n}$ other than $x_{\infty}$ on the boundary.

In fact for a $\sigma$-compact space $X$ with a compactification $\bar{X}$ having metrizable boundary, using the pseudo-metric $d_{\bar{X}}$ as constructed above, we can also construct a controlled neighbourhood of the diagonal in $X \times X$. The following lemma shows the construction.

Lemma 3.23. For $X$ a sigma-compact, locally compact, Hausdorff space, the topological coarse structure coming from a compactification $\bar{X}$ with metrizable boundary $\partial \bar{X}$ contains a controlled neighbourhood of the diagonal $\Delta_{X \times X}$.

Proof. Let $d_{\bar{X}}$ be the pseudo-metric we get by pulling back the metric on the boundary using the quotient map $r: \bar{X} \rightarrow \partial \bar{X}$ as above. Now consider the increasing union of compact sets $\left\{U_{n}\right\}_{n}$ in $X$. Then for every $x$ in $U_{n}$, consider the set $E_{n}:=\left\{(x, y) \in X \times X: d_{\bar{X}}(x, y)<2^{-n}\right\} . d_{\bar{X}}$ being continuous on $\bar{X} \times \bar{X}, E_{n}$ is open for every $n$. Consider the union $E=\cup_{n} E_{n}$. This is an open set that contains the diagonal since every $x$ in $X$ shall belong to some $U_{m}$ and hence $(x, x) \in E_{m} \subset E$. To show it is controlled, we need to show

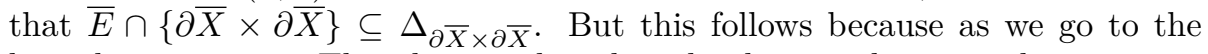
boundary, $n \rightarrow \infty$. This then implies that the distance between the points in $\bar{E} \cap\{\partial \bar{X} \times \partial \bar{X}\}$, which is given by $2^{-n}$, goes to 0 . 
The controlled sets defined for a topological coarse structure are always proper. This then proves that the topological coarse structure on a $\sigma$-compact space $X$ given by a compactification $\bar{X}$ with metrizable boundary, is a proper coarse structure.

Remark 3.24. In both Proposition 3.21 and Theorem 3.19 we can replace the assumption on the topological coarse structures to come from a second countable compactification by topological coarse structures on a $\sigma$-compact space given by a compactification with metrizable boundary. With Lemma 3.22 and Lemma 3.23 the same proof for Proposition 3.21 and Theorem 3.19 goes through for such broader class of coarse structures.

Next we look at the relation between coarse maps for topological coarse structures and the behaviour of such maps between the compactifications that define the coarse structure. This is in the same spirit as in Proposition 3.28, where Roe shows that for proper coarse structures a coarse map induces a continuous map between the Higson coronas. We show that for proper topological coarse structures defined by a compactification as above, a coarse map induces a continuous map between the boundaries of the compactification.

Lemma 3.25. Let $X, Y$ be $\sigma$-compact, locally compact Hausdorff spaces with topological coarse structures associated to compactifications $\bar{X}, \bar{Y}$ with metrizable boundaries $\partial \bar{X}, \partial \bar{Y}$ respectively. Then a coarse map $f: X \rightarrow Y$ extends to a man between the boundaries $\partial \bar{f}: \partial \bar{X} \rightarrow \partial \bar{Y}$.

Proof. From Lemma 3.22 given a point $x_{0}$ in $\partial \bar{X}$ we choose a sequence $\left\{x_{n}\right\}_{n}$ in $X$ that converges to $x_{0}$ and has no other accumulation point in $\bar{X}$. $f$ being proper and bornologous, for the controlled set $E:=\left\{\left(x_{n}, x_{n}\right)\right\}$, we have $f \times f(E)$ is also a controlled set in $(Y, \bar{Y})$. In particular $\left\{f\left(x_{n}\right)\right\}_{n}$ shall have an accumulation point $y_{0}$ at the boundary. Suppose that it has more than one accumulation points, say $y_{1}$ in the boundary $\partial \bar{Y}$. Then one can choose a subsequence $\left\{f\left(x_{k}\right)\right\}_{k} \subset\left\{f\left(x_{n}\right)\right\}_{n}$ such that $\left\{f\left(x_{k}\right)\right\}_{k}$ converges to $y_{1}$. But $f$ is bornologous. Then $\left\{x_{k}\right\}_{k}$ also goes out to the boundary $\partial \bar{X}$. Suppose not, then for any accumulation point $x$ of $\left\{x_{k}\right\}_{k}$ in $\bar{X}$ that lies in the interior of $X,\left\{\left(x_{k}, x\right)\right\}_{k}$ is a controlled set in $(X, \bar{X})$. Then $f \times f\left(\left\{\left(x_{k}, x\right)\right\}_{k}\right)$ should be a controlled set in $(Y, \bar{Y})$. By our assumption $\left\{f\left(x_{k}\right)\right\}_{k}$ converges to $y_{1}$ at the boundary $\partial \bar{Y}$. But $Y$ being locally compact, $f(x)$ lies inside a compact set in $Y$ and hence is in the interior of $Y$. Thus $f \times f\left(\left\{\left(x_{k}, x\right)\right\}_{k}\right)$ is not a controlled set and hence contradicts that $f$ is bornologous. Thus $\left\{x_{k}\right\}_{k}$ being a subsequence of the converging sequence $\left\{x_{n}\right\}_{n}$, it converges to $x_{0} \in \partial \bar{X}$. Now $\left\{f\left(x_{k}\right)\right\}_{k}$ converges to $y_{1} \neq y_{0}$ at the boundary $\partial \bar{Y}$. Then for the controlled set $E^{\prime}:=\left\{\left(x_{n}, x_{k}\right)\right\}_{n, k}$ we see that $f \times f\left(E^{\prime}\right)$ is not a controlled set in $(Y, \bar{Y})$. This then contradcts that $f$ is bornologous. Therefore we have that $\left\{f\left(x_{n}\right)\right\}_{n}$ shall have only a single accumulation point at the boundary and that $\left\{f\left(x_{k}\right)\right\}_{k}$ also converges to the same point $y_{0}$. Again for any other sequence $\left\{x_{m}^{\prime}\right\}_{m}$ that converges to $x_{0}$, taking the controlled set $E^{\prime}:=\left\{\left(x_{n}, x_{m}^{\prime}\right)\right\}_{n, m}$ and that $f$ is bornologous, we have that $\left\{f\left(x_{m}^{\prime}\right)\right\}_{m}$ also converges to the same point $y_{0}$. Thus we can define an extension of $f$ at the boundary by $\partial \bar{f}\left(x_{0}\right):=y_{0}$. Therefore

\footnotetext{
${ }^{3}$ In fact as shall be shown in Lemma 3.26 below, $\partial \bar{f}$ is continuous.

${ }^{4}$ There exist accumulation points of a net in a compact topological space.
} 
we can define an extension of $f$ at $x_{0}$ on the boundary by $\partial \bar{f}\left(x_{0}\right):=y_{0}$. Since $x_{0}$ chosen was arbitrary, we repeat this process for all $x_{0} \in \partial \bar{X}$ and thereby get a well-defined map between the boundaries induced by $f$. From Lemma 3.26 below we see that $\partial \bar{f}$ is continuous.

The definition of $\partial \bar{f}$ being an extension of $f$ says that for any $x_{0} \in \partial \bar{X}$ and any net $\left\{x_{\alpha}\right\}_{\alpha}$ converging to $x_{0},\left\{f\left(x_{\alpha}\right)\right\}_{\alpha}$ is also a convergent net and converges to a particular point on the boundary $\partial \bar{Y}$. The limit point of $\left\{f\left(x_{\alpha}\right)\right\}_{\alpha}$ should then be independent of the net $\left\{x_{\alpha}\right\}_{\alpha}$ chosen and determined only by the point $x_{0}$. Only then can we define a well-defined extension of $f$ on the boundary,

$$
\partial \bar{f}\left(x_{0}\right):=\lim _{x_{\alpha}} f\left(x_{\alpha}\right) .
$$

The limit point $\lim _{x_{\alpha}} f\left(x_{\alpha}\right)$ belongs to the boundary $\partial \bar{Y}$, is clear from $f$ being a proper map. For the map $\partial \bar{f}$ to be well-defined as an extension of the map $f$, we need the condition that for all nets converging to $x_{0}$ their image under $f$ should converge to the same limit point in $\partial \bar{Y}$. Interestingly, this condition would also make $\partial \bar{f}$ continuous between the boundaries.

Lemma 3.26. If a not necessarily continuous proper map $f: X \rightarrow Y$ extends to a well-defined map $\partial \bar{f}: \partial \bar{X} \rightarrow \partial \bar{Y}$ on the boundary, then $\partial \bar{f}$ is continuous.

Proof. Suppose $\partial \bar{f}$ is not continuous at $x_{0} \in \partial \bar{X}$. Then there exists a neighbourhood $U$ of $\partial \bar{f}\left(x_{0}\right)$ and a net $\left\{x_{\alpha}\right\}_{\alpha \in \mathcal{I}} \subset \partial \bar{X}$ such that $x_{\alpha} \rightarrow x_{0}$ but $\partial \bar{f}\left(x_{\alpha}\right) \notin U$ for all $\alpha \in \mathcal{I}$. Now $X$ being dense in $\bar{X}$ for all $x_{\alpha}$ in $\partial \bar{X}$, there exists a net $\left\{x_{\alpha, \beta}\right\}_{\beta \in I_{\alpha}}$ such that $x_{\alpha}=\lim _{\beta \in I_{\alpha}} x_{\alpha, \beta}$. Consider the directed set of neighbourhoods $\left\{V_{\gamma}\right\}_{\gamma}$ of $x_{0}$ in $\bar{X}$ ordered by inclusion, that is, $V_{\gamma_{1}} \geq V_{\gamma_{2}}$ if and only if $V_{\gamma_{1}} \subseteq V_{\gamma_{2}}$. Then from the given set $\left\{x_{\alpha, \beta}\right\}_{\alpha \in \mathcal{I}, \beta \in \mathcal{I}_{\underline{\alpha}}}$ choose points $x_{\gamma, \alpha}$ in $V_{\gamma}$. Since $\left\{x_{\alpha}\right\}_{\alpha} \subseteq \partial \bar{X}$ converges to $x_{0}$ and $X$ is dense in $\bar{X}$ we get a non-empty subnet $\left\{x_{\gamma, \alpha}\right\}$ that converges to $x_{0}$. But by our assumption of discontinuity of $\partial \bar{f}$ at $x_{0},\left\{f\left(x_{\gamma, \alpha}\right)\right\}$ cannot accumulate at $\partial \bar{f}\left(x_{0}\right)$. This is because $\bar{X}$ being normal, we can separate the two disjoint closed sets $A:=\partial \bar{f}\left(x_{0}\right)$ and $B:=\overline{\left\{\partial \bar{f}\left(x_{\alpha}\right)\right\}_{\alpha}}$ by disjoint neighbourhoods $U_{A}$ and $U_{B}$. Now the condition of extendability of $f$ to $\partial \bar{f}$ at the boundary dictates subnets in $\left\{f\left(x_{\alpha, \beta}\right)\right\}_{\alpha, \beta}$ to converge to points only in $\overline{\left\{\partial \bar{f}\left(x_{\alpha}\right)\right\}_{\alpha}}$. Thus for every $\alpha_{0}$ there exists $\beta_{0} \in \mathcal{I}_{\alpha_{0}}$ such that for all $\left(\alpha_{0}, \beta\right) \geq\left(\alpha_{0}, \beta_{0}\right), f\left(x_{\alpha_{0}, \beta}\right) \in U_{B}$. Thus $\left\{f\left(x_{\gamma, \alpha}\right)\right\}_{\gamma, \alpha} \subset\left\{f\left(x_{\alpha, \beta}\right)\right\}$ cannot accumulate at $\partial \bar{f}\left(x_{0}\right)$ even though $\left\{x_{\gamma, \alpha}\right\} \rightarrow x_{0}$. This contradicts that $f$ extends to a well-defined map $\partial \bar{f}$ at $x_{0}$.

Next for two coarse maps $f, g: X \rightarrow Y$, we show that the two maps being close is equivalent to the induced boundary maps being the same. Note that this is in the same spirit as the second result in Proposition 3.28 .

Lemma 3.27. Let $X, Y$ be $\sigma$-compact, locally compact and Hausdorff topological spaces with coarse structure associated to compactifications with metrizable boundaries $\bar{X}, \bar{Y}$ respectively. Then two coarse maps $f, g: X \rightarrow Y$ are close if and only if they induce the same map between the boundaries.

Proof. Given $f, g: X \rightarrow Y$ are close coarse maps, $E:=\{(f(x), g(x)): x \in X\}$ is a controlled set in $Y$. By Lemma 3.22 for every $x_{0} \in \partial \bar{X}$, there exists a 
sequence $\left\{x_{n}\right\}_{n}$ that converges to $x_{0}$. But then $f, g$ being coarse maps we have by Lemma 3.25 that $\left\{f\left(x_{n}\right)\right\}_{n},\left\{g\left(x_{n}\right)\right\}_{n}$ should also converge to certain boundary points $y_{0}=\partial \bar{f}\left(x_{0}\right)$ and $y_{0}^{\prime}=\partial \bar{g}\left(x_{0}\right)$ in $\partial \bar{Y}$ respectively. Now the set $\left\{\left(f\left(x_{n}\right), g\left(x_{n}\right)\right)\right\}_{n}$ is a subset of $E$ and hence is a controlled set in $(Y, \bar{Y})$. This then means that $y_{0}=y_{0}^{\prime}$. But then since $x_{0}$ chosen is arbitrary, this would mean that for every $x_{0} \in \partial \bar{X}, \partial \bar{f}\left(x_{0}\right)=\partial \bar{g}\left(x_{0}\right)$. Therefore the maps induced at the boundary by the close coarse maps $f, g$ are the same. For the converse direction, $f, g$ being bornologous, interior points of $X$ shall be mapped in the interior of $Y$. Otherwise if a net $\left\{x_{\alpha}\right\}_{\alpha}$ goes out to the boundary $\partial \bar{X}, \partial \bar{f}=\partial \bar{g}$ implies $\left\{\left(f\left(x_{\alpha}\right),\left(g\left(x_{\alpha}\right)\right)\right\}_{\alpha}\right.$ shall have accumulation points in $\Delta_{\partial \bar{Y} \times \partial \bar{Y}}$. Hence $\{(f(x), g(x)): x \in X\}$ is a controlled set in $(Y, \bar{Y})$ and therefore $f$ is close to $g$.

We next consider the Higson corona, a very important object as a functor from the category of proper coarse structures with coarse maps as morphisms to the category of compact Hausdorff topological spaces with continuous maps between them.

\subsubsection{Functoriality of the Higson corona}

Recall the definition of Higson compactification corresponding to a proper coarse structure $(X, \mathcal{E})$ on a locally compact Hausdorff space $X$, as in Chapter 1 The Higson condition of vanishing variation on functions, as in Definition 1.5. has a very useful outcome on the behaviour of such functions at the boundary. Namely, this allows the algebra of continuous Higson functions $C_{h}(X)$ on $X$ and the algebra of not necessarily continuous (but Borel measurable ${ }^{5}$ ) Higson functions $B_{h}(X)$ to be isomorphic when restricted to their respective boundaries. Thus $C_{h}(X) / C_{0}(X) \cong B_{h}(X) / B_{0}(X)$ will say that they have homeomorphic boundaries. Using this isomorphism, Roe establishes functoriality of the Higson corona $\nu X$ even with respect to discontinuous coarse maps $f: X \rightarrow Y$.

Proposition 3.28. 9, Proposition 2.41] Let $X$ and $Y$ be proper coarse spaces. Then a coarse map $f: X \rightarrow Y$ extends to a continuous map $\nu f: \nu X \rightarrow \nu Y$. If $f, g: X \rightarrow Y$ are close then $\nu f=\nu g$.

The next corollary automatically follows from the definition of coarse equivalence and closeness of maps.

Corollary 3.29. A coarse equivalence induces a homeomorphism between the Higson corona.

Let us next study the $*$-homomorphism induced by a coarse map between the Higson corona algebras, as implied by Proposition 3.28 above. A proper coarse structure requires the underlying topological space to be paracompact. Hence there exists continuous partition of unity subordinate to any given open cover of

\footnotetext{
${ }^{5}$ since one needs to make sense of a continuous partition of unity argument to go from $B_{h}(X)$ to $C_{h}(X)$

${ }^{6} B_{0}(X)$ stands for the algebra of Borel measurable functions on $X$ that tends to 0 as one approaches the boundary.
} 
$X$. Of particular interest is a uniformly bounded open cover of $X$, coming from the controlled neighbourhood $E$ of the diagonal for the proper coarse structure on $X$. The details of this construction can be seen below within the proof of Lemma 3.34

The idea is then to define a completely positive map between the $\mathrm{C}^{*}$-algebras using a certain continuous partition of unity. Certain additional conditions are to be satisfied in the choice of this partition of unity, with which the completely positive map shall then restrict to a $*$-homomorphism between the Higson corona algebras. Finally, one shows that the construction is independent of the choice of the partition of unity as long as they satisfy the conditions stated.

We begin with an abstract definition of these additional conditions, we referred to in the previous paragraph.

Definition 3.30. Given $X$ as a coarse space, a regularizer $P$ for $X$ is a linear map from the space of $\omega$-bounded Borel functions on $X$ to the space of continuous functions on $X$, satisfying the properties

1. If $\phi \geq 0$, then $P(\phi) \geq 0$.

2. $P(1)=1$.

3. There is a constant $r>0$ such that for any $x \in X$ and any function $g$ on $X$, the value of $P(g)(x)$ depends only on the restriction of $g$ to $B(x ; r)$, the ball of radius $r$ around $x$.

For the bounded metric coarse structure on a proper metric space $(X, d)$, such regularizers can be constructed from $\left\{\psi_{i}\right\}_{i}$, a continuous partition of unity subordinate to a locally finite cover of $X$ by balls $B\left(x_{i} ; r\right)$ of fixed radius $r>0$, by defining

$$
P(g)(x)=\Sigma_{i} g\left(x_{i}\right) \psi_{i}(x)
$$

Using such regularizers, Roe shows the following.

Proposition 3.31. 8, Proposition 5.10] Given $X$ a proper metric coarse space with a not necessarily continuous coarse map $f: X \rightarrow Y$ for a coarse space $Y$. Then for any regularizer $P$ on $X$, the map $f^{*}:[g] \mapsto[P(g \circ f)]^{7}$ is a well-defined homomorphism of the Higson corona algebras $C(\nu Y) \rightarrow C(\nu X)$. Moreover, it is independent of the choice of $P$.

In the case of a proper topological coarse structures on $X$ coming from a compactification $\bar{X}$, we choose $E[x]$ instead of the ball $B(x ; r)$ where $E$ is a controlled neighbourhood of the diagonal of the proper coarse structure. Then the continuous partition of unity is chosen subordinate to a locally finite refinement of the uniformly bounded open cover of $X$ coming from $E$. Using Proposition 3.21 . a coarse map on $X$ shall then induce a similar $*$-homomorphism between the boundary quotient algebras.

It is important to keep in mind that only proper coarse structures are relevant for $\mathrm{C}^{*}$-algebraic study. In the next section we shall treat the construction of

\footnotetext{
${ }^{7}$ The square brackets denote equivalence classes in the quotient algebras.
} 
regularizers in more details for the broader class of all proper coarse structures coming from a compactification. Then by the above construction, starting with a coarse map on a proper coarse space, we expect to arrive at similar completely positive maps that restrict to a $*$-homomorphism between the boundary quotient algebras. This would then justify to a certain extent our definition of a noncommutative coarse map.

\subsubsection{Discontinuous coarse maps for topological coarse struc- tures}

The main perspective of this section, is to bridge the gap in the understanding of the definition behind a noncommutative coarse map in the commutative setting. Therefore we shall only be interested in topological coarse structures defined uniquely by compactifications, the path we have used to define a noncommutative coarse structure at the first place.

First, let us try to understand what would the converse direction to Proposition 3.28 above, possibly say. It would mean that we should be able to construct a coarse map from scratch by starting with a continuous map between the boundaries. We are still to find results in this direction.

Rather, we reformulate it such that a proper map $f: X \rightarrow Y$ between the spaces is already given. Now, with coarse structure coming from the compactifications $\bar{X}, \bar{Y}$, we ask if extending $f$ to a map $\partial \bar{f}: \partial \bar{X} \rightarrow \partial \bar{Y}$ between the boundaries imply that $f$ is coarse ?

Given a proper map $f: X \rightarrow Y$ between topological coarse spaces, that extends to a continuous map between the boundaries, we try to show that $f$ is bornologous. When $f$ is continuous, Remark 3.24 shows that by Theorem 3.19 this holds for $\sigma$-compact spaces with proper topological coarse structures defined by compactifications with metrizable boundaries. In the absence of continuity, $f$ being a proper map is not sufficient to guarantee that nets in $X$ with accumulation points in the interior of $X$ shall be mapped to a net in the interior of $Y$ without any accumulation points at the boundary $\partial \bar{Y}$. For this we need an extra condition on $f$ to be able to show that it is bornologous.

Definition 3.32. Let $f: X \rightarrow Y$ be a map between locally compact Hausdorff topological spaces with proper coarse structures. Then $f$ is said to be bounded if it maps all bounded subsets of $X$ to bounded subsets in $Y$.

Proposition 3.33. Let $f: X \rightarrow Y$ be a proper map between locally compact Hausdorff spaces $X, Y$ with proper topological coarse structures defined by compactifications $\bar{X}, \bar{Y}$ respectively. Suppose that $f: X \rightarrow Y$ is bounded and that it extends to a continuous map $\partial \bar{f}: \partial \bar{X} \rightarrow \partial \bar{Y}$ between the boundaries. Then $f$ is bornologous and hence a coarse map.

Proof. Given a proper map $f: X \rightarrow Y$ that extends to a continuous map $\partial \bar{f}: \partial \bar{X} \rightarrow \partial \bar{Y}$ we need to show that it maps controlled sets in $(X, \bar{X})$ to controlled sets in $(Y, \bar{Y})$. Now by the definition that $f$ extends to $\partial \bar{f}$ between the boundaries, every convergent net $\left\{x_{\alpha}\right\}_{\alpha}$ converging to a boundary point, say $x_{0}$, is mapped to a net $\left\{f\left(x_{\alpha}\right)\right\}_{\alpha}$ that converges to $\partial \bar{f}\left(x_{0}\right)$. But $f: X \rightarrow Y$ 
need not be a continuous map. By assuming that $f$ is bounded, for any net $\left\{x_{\alpha}\right\}_{\alpha}$ that accumulates at points in the interior of $X,\left\{f\left(x_{\alpha}\right)\right\}_{\alpha}$ cannot have accumulation points at the boundary $\partial \bar{Y}$. Hence by the definition of controlled sets coming from a compactification, every controlled set in $X$ is mapped to a controlled set in $Y$. Therefore $f$ is bornologous and hence a coarse map.

Next, we would like to motivate the use of completely positive maps in our definition of a noncommutative coarse map. Let us look back at the conditions required by a continuous map $f: X \rightarrow Y$ to be coarse with respect to topological coarse structures coming from second countable compactifications $\bar{X}, \bar{Y}$, as in Theorem 3.19 In the case of a countable discrete space $X$, this then leads to an interesting observation. Since every map on a discrete space is continuous, any proper map $f: X \rightarrow Y$ induces the $*$-homomorphism $f^{*}: C_{0}(Y) \rightarrow C_{0}(X)$. Then by Theorem $3.19, f$ is coarse if and only if it induces a $*$-homomorphism $(\bar{f})^{*}: C(\bar{Y}) \rightarrow C(\bar{X})$ between the unitizations.

As was mentioned, for $\mathrm{C}^{*}$-algebraic study we require compatibility of the coarse structure with the underlying topology as in a proper coarse space. Proper coarse spaces have an important property as we show in the following lemma.

Lemma 3.34. Every proper coarse space $X$ is coarsely equivalent to a discrete subspace $X_{\text {disc }}$.

Proof. To show this, consider the open neighbourhood $E$ of the diagonal $\Delta_{X \times X}$ which is also a controlled set by the requirements of a proper coarse structure. Then for every point $x$ in $X$, we have an open set $U_{x} \times V_{x} \subset E$ containing the point $(x, x)$. Let $W_{x}:=U_{x} \cap V_{x}$. Then clearly, $\left\{W_{x}\right\}_{x \in X}$ forms a uniformly bounded open cover of $X$. We next use that $X$ is paracompact, to choose a locally finite refinement of this open cover, say $\left\{W_{\alpha}\right\}_{\alpha \in \mathcal{J}}$, for an indexing set $\mathcal{J}$. Finally, choose any arbitrary point $x_{\alpha}$ from this open cover to construct $X_{\text {disc }}:=\left\{x_{\alpha}\right\}_{\alpha \in \mathcal{J}}$. Then by the cover being locally finite on a Hausdorff space, with the subspace topology $X_{\text {disc }}$ is a discrete subspace of $X$.

We claim that the inclusion map $i: X_{\text {disc }} \rightarrow X$ gives a coarse equivalence. For this it is trivial to see that the set $W:=\cup_{\alpha \in \mathcal{J}}\left\{x_{\alpha} \times W_{\alpha}\right\}_{\alpha} \subset E$ is a controlled set. Further $W\left[X_{\text {disc }}\right]=X$, which shows that $X_{\text {disc }}$ is coarsely dense in $X$. Hence to choose the inverse $i^{-1}$ to the inclusion map for the coarse equivalence, consider for any $x$ in $X$ the index $\alpha^{x} \in \mathcal{J}$, such that $x \in W_{\alpha^{x}}$. Then the following map $x \mapsto x_{\alpha^{x}}$ from $X$ to $X_{\text {disc }}$ shall give the required inverse coarse map for the coarse equivalence.

Remark 3.35. In explicit examples of proper coarse structures on a locally compact space that are uniquely determined by a compactification, $X$ is always $\sigma$-compact. A proper metric space with the bounded metric coarse structure is a proper coarse space and is uniquely determined by its Higson compactification. Otherwise, as in Remark 3.24 a $\sigma$-compact space with the topological coarse structure given by a compactification $\bar{X}$ with metrizable boundary is a proper coarse structure that is uniquely determined by the compactification. In 
Lemma 3.34 , this would lead to the choice of a countable discrete subspace $X_{\text {disc }}$ that is coarsely equivalent to $X$.

Given $X$ is a proper coarse space, from a coarse map $f: X \rightarrow Y$ we next construct a completely positive map between the $\mathrm{C}^{*}$-algebras. The idea is to restrict $f$ to a coarsely equivalent discrete subset $X_{\text {disc }} \subset X$ obtained by using a locally finite uniformly bounded open cover of $X$, as in Lemma 3.34 above. Then $\left.f\right|_{X_{\text {disc }}}: X_{\text {disc }} \rightarrow Y$ is a continuous coarse map and hence shall induce a *-homomorphism between the $\mathrm{C}^{*}$-algebras $\left(\left.f\right|_{X_{\text {disc }}}\right)^{*}: C_{0}(Y) \rightarrow C_{0}\left(X_{\text {disc }}\right)$.

Given that $X$ is a proper coarse space and hence paracompact, we use a continuous partition of unity $\left\{\phi_{\alpha}\right\}_{\alpha}$ subordinate to the locally finite open cover from which the discrete set $X_{\text {disc }}$ was extracted. Finally, the completely positive map $\phi: C_{0}\left(X_{\text {disc }}\right) \rightarrow C_{0}(X)$ is defined as

$$
\phi(g)(x):=\Sigma_{\alpha} g\left(x_{\alpha}\right) \phi_{\alpha}(x) ; \quad \forall g \in C_{0}\left(X_{\text {disc }}\right) .
$$

Then by composing with $\left(\left.f\right|_{X_{\text {disc }}}\right)^{*}$, we get a completely positive map $\phi \circ$ $\left(\left.f\right|_{X_{\mathrm{disc}}}\right)^{*}: C_{0}(Y) \rightarrow C_{0}(X)$.

Our next claim shall be that this construction of the completely positive map $\phi \circ\left(\left.f\right|_{X_{\text {disc }}}\right)^{*}: C_{0}(Y) \rightarrow C_{0}(X)$ do not change the limiting behaviour of $f$ at the boundaries. We expect a coarse map to induce a $*$-homomorphism between the boundary quotient algebras. We see later that by extending this construction to the unitizations, by Proposition 3.37 the completely positive map $\phi \circ\left(\left.f\right|_{X_{\text {disc }}}\right)^{*}: C_{0}(Y) \rightarrow C_{0}(X)$ does give the same $*$-homomorphism between the boundary quotient algebras as the one induced by $f$. But before that we show that the map $\partial\left(\overline{\left.f\right|_{X_{\text {disc }}}}\right)$ defined as an extension of the restriction map $\overline{\left.f\right|_{X_{\text {disc }}}}$ on the discrete space to the boundary is the same as $\partial \bar{f}$. For this, we first note that Corollary 3.29 , gives a homeomorphism between the boundaries $\partial \overline{X_{\text {disc }}}$ and $\partial \bar{X}$.

Lemma 3.36. Given $X$ is coarsely equivalent to a discrete subset $X_{\text {disc }}$, if $f: X \rightarrow Y$ is a coarse map then there exists an extension of $\left.f\right|_{X_{\text {disc }}}$ to a continuous map on the boundary $\partial\left(\overline{\left.f\right|_{X_{\mathrm{disc}}}}\right): \partial \overline{X_{\mathrm{diss}}} 8 \rightarrow \partial \bar{Y}$. Moreover $\partial\left(\overline{\left.f\right|_{X_{\mathrm{disc}}}}\right)=\partial \bar{f}$.

Proof. $f: X \rightarrow Y$ is a coarse map. This implies from Lemma 3.25 that there exists an extension of $f$ to a continuous map between the boundaries $\partial \bar{f}: \partial \bar{X} \rightarrow$ $\partial \bar{Y}$. But the boundary $\partial \overline{X_{\text {disc }}}$ is homeomorphic to $\partial \bar{X}$. Then for every point $x_{0}$ at the boundary $\partial \bar{X}$, there exists a net in $X_{\text {disc }}$ converging to $x_{0}$. For $\left\{x_{\alpha}\right\}_{\alpha}$ in $X_{\text {disc }} \subset X$ converging to a point $x_{0}$ at the boundary $\partial \bar{X}$, we have from the definition of $\partial \bar{f}$ that $\left\{f\left(x_{\alpha}\right)\right\}_{\alpha}$ is a convergent net, converging to the point $\partial \bar{f}\left(x_{0}\right)$ in $\partial \bar{Y}$. Hence with this we define the map $\partial\left(\overline{\left.f\right|_{X_{\mathrm{disc}}}}\right): \partial \bar{X} \rightarrow \partial \bar{Y}$ as an extension of $\left.f\right|_{\text {disc }}$ and satisfying trivially the condition $\partial\left(\overline{\left.f\right|_{X_{\text {disc }}}}\right)=\partial \bar{f}$ by its very definition.

Together with this Lemma, we can then show that $i^{-1} \circ\left(\left.f\right|_{X_{\text {disc }}}\right): X \rightarrow Y$ shall induce a $*$-homomorphism between the boundaries, same as $(\partial \bar{f})^{*}$. We

\footnotetext{
${ }^{8} \partial \overline{X_{\text {disc }}}=\partial \bar{X}$. From the construction of the discrete set we know for each point $\omega \in \partial \bar{X}$, there exists a net in $X_{\text {disc }}$ converging to it. Therefore $\partial \bar{X} \subset \partial \overline{X_{\text {disc }}}$. The other inclusion follows from $X_{\text {disc }}$ being a subset of $X$ with the subspace topology.
} 
have used here the map $i^{-1}$, to show the requirement of a $*$-homomorphism between the boundary quotient algebras and havent used $\phi$ explicitly yet. The main obstruction is that $i^{-1}$ need not be continuous, and hence we cannot induce a map between the $\mathrm{C}^{*}$-algebras, $C_{0}\left(X_{\text {disc }}\right) \rightarrow C_{0}(X)$, though we get a *homomorphism $\partial\left(i^{-1}\right)^{*}$ between the boundary quotient $\mathrm{C}^{*}$-algebras. We show next how we use our construction of the map $\phi$ to bridge this gap.

The cover $\left\{W_{\alpha}\right\}_{\alpha \in \mathcal{J}}$ chosen to construct $\phi$ above is locally finite. Hence $\phi$ is strictly continuous. We have that for each $x \in X$ the open neighbourhood $U_{x} \cap V_{x}$ is a subset of $E[x]$. Therefore $\left\{\left(x_{\beta}, x_{\beta}\right)\right\}_{\beta}$ should only have $\left(x_{0}, x_{0}\right)$ as its accumulation point as $\left\{x_{\beta}\right\}_{\beta}$ converges to $x_{0} \in \partial \bar{X}$. But $\left\{W_{\alpha}\right\}_{\alpha \in \mathcal{J}}$ being a refinement of $\left\{U_{x} \cap V_{x}\right\}_{x \in X}$, it should also have similar behaviour. Also the points $\left\{x_{\alpha}\right\}_{\alpha}$ chosen to define $X_{\text {disc }}$ shall behave similarly as one approaches the boundary. Therefore the continuous partition of unity $\left\{\phi_{\alpha}\right\}_{\alpha}$ chosen to construct $\phi$, being subordinate to this open cover, will give the identity $*$ isomorphism between the boundary quotient algebras.

Next by using that $\phi$ is strictly continuous, we extend the map $\phi$ to the unitizations $\bar{\phi}: C\left(\overline{X_{\text {disc }}}\right) \rightarrow C(\bar{X})$ with nice behaviour between the boundary quotient algebras.

Proposition 3.37. Let $\phi: C_{0}\left(X_{\mathrm{disc}}\right) \rightarrow C_{0}(X)$ be the completely positive map as in equation 3.1. Then it extends to a completely positive map between the unitizations $\bar{\phi}: C\left(\overline{X_{\mathrm{disc}}}\right) \rightarrow C(\bar{X})$ such that it induces the identity $*$-isomorphism between the continuous functions on the boundary.

Proof. Let us consider the completely positive map $\phi: C_{0}\left(X_{\text {disc }}\right) \rightarrow C_{0}(X)$ we have defined before,

$$
\phi(g)(x):=\Sigma_{\alpha} g\left(x_{\alpha}\right) \phi_{\alpha}(x) ; \quad \forall g \in C_{0}\left(X_{\text {disc }}\right) .
$$

We had noted before that $\left\{W_{\alpha}\right\}_{\alpha}$ being a locally finite cover, $\phi$ is strictly continuous. Now the functions $\phi_{\alpha}$ all belong to $C_{0}(X)$. But $C_{0}(X)$ being dense in $C_{b}(X)$ with respect to the strict topology, for any $g \in C_{b}(X)$ we have a net $\left\{g_{\beta}\right\}_{\beta}$ in $C_{0}(X)$ converging to it in the strict topology. This implies for all $\alpha$ in the indexing set $\mathcal{J},\left\|g_{\beta} \phi_{\alpha}-g \phi_{\alpha}\right\|_{C_{0}(X)} \rightarrow 0$ in the limit of $g_{\beta}$ converging to $g$ in strict topology. For a given point $x \in X$, we can consider equation 3.1 as the following

$$
\begin{aligned}
\phi(g)(x) & =\Sigma_{\alpha} g\left(x_{\alpha}\right) \phi_{\alpha}(x) \\
& =\Sigma_{\alpha} g\left(x_{\alpha}\right) \phi_{\alpha}\left(x_{\alpha}\right) \phi_{\alpha}(x) .
\end{aligned}
$$

The second equality follows from $\phi_{\alpha}\left(x_{\beta}\right)=\delta_{\alpha, \beta}$, the Kronecker delta function, for all $x_{\beta} \in X_{\text {disc }}$. For a fixed $x$ in $X$, this can then be seen as integration of the functions $g \phi_{\alpha}$ over the elements $x_{\alpha} \in X_{\text {disc }}$ with respect to the positive, weighted counting measure $\phi_{\alpha}(x)$ on $\left\{x_{\alpha}\right\}_{\alpha \in \mathcal{J}}$. Further the open cover being locally finite, we have that for any given $x \in X$ only finitely many indices $\alpha \in \mathcal{J}$ shall give non-zero contribution. But finitely many indices define a compact set in the discrete space $X_{\text {disc }}$. Hence using uniform convergence of $\left\|g_{\beta} \phi_{\alpha}-g \phi_{\alpha}\right\|_{C_{0}(X)} \rightarrow 0$ in $C_{0}(X)$, we can use the same estimates when restricted to $C_{0}\left(X_{\text {disc }}\right)$, to interchange between the limit and the integration. Thus for all 
$g \in C_{b}(X)$, at a fixed point $x \in X$, the following convergent net $\phi\left(g_{\beta}\right)(x)=$ $\Sigma_{\alpha} g_{\beta}\left(x_{\alpha}\right) \phi_{\alpha}\left(x_{\alpha}\right) \phi_{\alpha}(x)$ converges to $\Sigma_{\alpha} g\left(x_{\alpha}\right) \phi_{\alpha}(x)$.

With this we define the extension of the completely positive map

$$
\bar{\phi}(g)(x)=\Sigma_{\alpha} g\left(x_{\alpha}\right) \phi_{\alpha}(x) ; \quad \forall g \in C(\bar{X}) .
$$

It is now clearly visible that as $x$ converges to $x_{0}$ at the boundary $\partial \bar{X}$, since the choice of $x_{\alpha}$ means that $\left(x, x_{\alpha}\right)$ belongs to the controlled set $E$, we have $x_{\alpha}$ also converges to the same boundary point $x_{0} \in \partial \bar{X} 9$ Hence equation 3.2 shall then say that for $g \in C(\bar{X})$, and a net $\left\{x_{\gamma}\right\}_{\gamma} \subset X_{\text {disc }}$ converging to $x_{0}$, we have from the continuity of the functions $\phi_{\alpha}$ used to define the partition of unity, that $\bar{\phi}(g)\left(x_{0}\right)=\lim _{x_{\gamma} \rightarrow x_{0}} \bar{\phi}(g)\left(x_{\gamma}\right)$. But $\bar{\phi}(g)\left(x_{\gamma}\right)=\Sigma_{\alpha} g\left(x_{\alpha}\right) \phi_{\alpha}\left(x_{\gamma}\right)=$ $g\left(x_{\gamma}\right)$. Hence, $\lim _{x_{\gamma} \rightarrow x_{0}} \bar{\phi}(g)\left(x_{\gamma}\right)$ is equal to $\lim _{x_{\gamma} \rightarrow x_{0}} g\left(x_{\gamma}\right)=g\left(x_{0}\right)$. Therefore $\bar{\phi}(g)\left(x_{0}\right)=g\left(x_{0}\right){ }^{10}$, for all $g \in C(\bar{X})$ and all $x_{0} \in \partial \bar{X}$.

With this we have that our construction of the completely positive map $\phi$ o $\left(\left.f\right|_{X_{\text {disc }}}\right)^{*}: C_{0}(Y) \rightarrow C_{0}(X)$ do not change the boundary $*$-homomorphism induced by the original coarse map $f: X \rightarrow Y$.

Finally we consider the equivalence relation of closeness, up to which coarse maps are defined. Recall from Lemma 3.27that for topological coarse structures two coarse maps are close if and only if they extend to the same map between the boundaries. As we will see later we define closeness for noncommutative coarse maps using this condition. This also dictates our notion of a noncommutative coarse equivalence.

\subsubsection{More coarse maps}

The main point is that, in the commutative case, we could show so far that the conditions on a noncommutative coarse map are only necessary for being induced by a coarse map $f: X \rightarrow Y$. We could not prove that they are also sufficient to be induced by a coarse map. Namely, we would need to show that starting with a contractive, strictly continuous completely positive map that satisfies the conditions of a noncommutative coarse map, its restriction to the pure state space is close to a honest coarse map. Only once this is showed, we can consider our definition of a noncommutative coarse map to be complete from the viewpoint of coarse geometry.

For this we need to be able to construct a point-valued map from a probability measure-valued map, with the boundary condition that the completely positive map $\phi \circ\left(\left.f\right|_{X_{\text {disc }}}\right)^{*}: C_{0}(Y) \rightarrow C_{0}(X)$ should induce the same map as $i^{-1} \circ\left(\left.f\right|_{X_{\text {disc }}}\right)$ between the boundaries $\partial \bar{X} \rightarrow \partial \bar{Y}$. To do this it should be enough to be able to appropriately choose points from the support of the measures. This will then say that the completely positive map $\phi \circ\left(\left.f\right|_{X_{\text {disc }}}\right)^{*}: C_{0}(Y) \rightarrow C_{0}(X)$ is equivalent to the coarse map $i^{-1} \circ\left(\left.f\right|_{X_{\text {disc }}}\right)$, which is again close to $f$ by Lemma 3.36

\footnotetext{
${ }^{9} \partial \overline{X_{\text {disc }}}=\partial \bar{X}$ by Corollary 3.29

${ }^{10}$ The function $g$ on the left hand side, that is the one in the domain of the map $\bar{\phi}$ is the restriction of $g \in C(\bar{X})$ to $\overline{X_{\text {disc }}}$, recall $\bar{\phi}: C\left(\overline{X_{\text {disc }}}\right) \rightarrow C(\bar{X})$.
} 
But there is no obvious natural choice for this construction, so we keep it open for now. The condition that the unital strictly continuous completely positive map should restrict to a $*$-homomorphism between the boundaries only say that the measures should converge to points as we approach the boundary. But it can still happen that even with this convergence condition on the measures, their support is large enough to have multiple accumulation points on the boundary. We expect that choosing a point-valued coarse map from a given noncommutative coarse map shall follow from extra conditions on our setting. We need more examples to motivate its importance. Until then, our definition of a noncommutative coarse map shall contain more maps as coarse maps in the commutative case.

\subsection{Noncommutative coarse equivalence}

As has already been mentioned many times by now, motivated from insights coming from physics, one of the main objectives for this project has been to formulate a possible coarse equivalence between the classical plane and the Moyal plane. We have already understood to a certain extent the notion behind a noncommutative coarse map. Following coarse equivalence in classical coarse geometry, we then define the corresponding notion for noncommutative coarse structures.

First let us consider the equivalence relation of closeness between two noncommutative coarse maps. Since noncommutative coarse maps generalize the notion of coarse maps between topological coarse structures, from Lemma 3.27 it is natural to consider the following definition of closeness between two noncommutative coarse maps.

Definition 3.38. Let $A, B$ be non-unital $\mathrm{C}^{*}$-algebras with noncommutative coarse structures defined by the unitizations $\bar{A}, \bar{B}$ respectively. Two noncommutative coarse maps $\phi, \psi: A \rightarrow B$ are called close if their extensions $\bar{\phi}, \bar{\psi}: \bar{A} \rightarrow$ $\bar{B}$ induce the same $*$-homomorphism between the boundary quotient algebras, that is $[\bar{\phi}]=[\bar{\psi}]$.

Remark 3.39. Clearly this defines a equivalence relation on the set of noncommutative coarse maps between the $\mathrm{C}^{*}$-algebras $A, B$ with noncommutative coarse structures fixed by certain unitizations.

Definition 3.40. Let $A, B$ be non-unital $\mathrm{C}^{*}$-algebras with noncommutative coarse structures defined by the following unitizations $\bar{A}, \bar{B}$. Then a noncommutative coarse map $\phi: A \rightarrow B$ defines a noncommutative coarse equivalence if the map $[\bar{\phi}]: \bar{A} / A \rightarrow \bar{B} / B$ is a $*$-isomorphism and there exists a noncommutative coarse map $\psi: B \rightarrow A$ in the opposite direction, such that $[\bar{\psi}]: \bar{B} / B \rightarrow \bar{A} / A$ induces the inverse $*$-isomorphism between the boundary quotient algebras.

Given this, it would be instructive to see that for any non-unital and $\sigma$-unital $\mathrm{C}^{*}$-algebra $B$, the unitalization $B^{\dagger}$ induces a unique noncommutative coarse structure. By uniqueness we mean upto noncommutative coarse equivalence. To see this we show coarse equivalence of $\left(B, B^{\dagger}\right)$ with $\left(C_{0}([0,1)), C_{0}([0,1])\right)$. Then 
using transitivity of this equivalence relation, we know that all noncommutative coarse structures induced by the smallest unitization $B^{\dagger}$ on a $\sigma$-unital $B$ are coarsely equivalent in the above sense.

Example 3.41. We use coarse equivalence of $[0,1)$ with $\mathbb{N}$ given by the inclusion of the discrete space, as was mentioned after Definition 3.18. Then we show that for any non-unital and $\sigma$-unital $\mathrm{C}^{*}$-algebra $B$ with noncommutative coarse structure given by $B^{\dagger}$, there exists a noncommutative coarse map $\phi: C_{0}([0,1)) \rightarrow B$ that induces a noncommutative coarse equivalence with the coarse structure on $C_{0}([0,1))$ coming from the unitization $C([0,1])$. For any strictly positive element $b \in B$, using functional calculus consider the following *-homomorphism

$$
\begin{aligned}
\phi: C_{0}([0,1)) & \rightarrow B \\
f & \mapsto f(b)
\end{aligned}
$$

This is clearly strictly continuous and induces the identity map id: $\mathbb{C} \rightarrow \mathbb{C}$ between the boundary quotient algebras. To get the inverse map, consider the completely positive map $\psi: B \rightarrow C_{0}(\mathbb{N})$ defined by the following construction. Consider the sequence of states $\left\{\psi_{n}\right\}_{n}$ on $B$ such that for all $b \in B, \psi_{n}(b) \rightarrow 0$ as $n \rightarrow \infty$. Then the completely positive map $\psi:=\left(\psi_{n}\right)$ such that for any $b$ in $B$, $\psi(b): n \mapsto \psi_{n}(b)$ an element of $C_{0}(\mathbb{N})$, gives the required noncommutative coarse inverse to $\phi$. Finally we use the coarse equivalence map of $C_{0}(\mathbb{N}) \rightarrow C_{0}([0,1))$ to get the required noncommutative coarse equivalence of $\left(C_{0}([0,1)), C_{0}([0,1])\right)$ with $\left(B, B^{\dagger}\right)$.

Let $Y$ be a proper metric space $\left(Y, d_{Y}\right)$ with the bounded metric coarse structure coming from $d_{Y}$. Then there is a further reduction on the definition of a coarse equivalence. We consider $X$ with a proper topological coarse structure coming from some compactification $\bar{X}$. Then if a coarse map $f: X \rightarrow Y$ induces a homeomorphism between the boundaries, we show that it gives a coarse equivalence between $X$ and $Y$.

Proposition 3.42. Let $\left(Y, d_{Y}\right)$ be a proper metric space with the bounded metric coarse structure coming from $d_{Y}$ with Higson compactification $\bar{Y}$. Let $X$ be a locally compact Hausdorff space with a proper coarse structure given by some compactification $\bar{X}$. If $f: X \rightarrow Y$ is a coarse map that extends to a homeomorphism $\partial \bar{f}: \partial \bar{X} \rightarrow \partial \bar{Y}$ between the boundaries, then $f$ is a coarse equivalence.

Proof. We first show that if the coarse map $f: X \rightarrow Y$ extends to a homeomorphism between the boundaries, then the image of this map $f(X)$ should be coarsely dense in $Y$. In other words, there should be a controlled set $E$ such that $E[f(X)]=Y$. Suppose not, then for every controlled set $E_{r} ; r>0$ there exists $y_{r}$ in $Y$ such that for all $x$ in $X, d_{Y}\left(y_{r}, f(x)\right)>r$. But then the collection $\left\{y_{r}\right\}_{r}$ shall have an accumulation point, say $y_{\infty}$ at the boundary. An explicit construction of the accumulation point $y_{\infty}$ can be shown as follows. Consider $F_{r}:=\operatorname{cls}\left\{x_{i}: i \geq r\right\}$, where "cls" refers to the closed linear span in $\bar{Y}$. Then it is easy to see that $\left\{F_{r}\right\}_{r}$ has the finite intersection property. Next $\bar{Y}$ being compact, we see that $\cap_{r} F_{r}$ should be non-empty inside $\bar{Y}$. Pick $y_{\infty}$ from $\cap_{r} F_{r}$. 
Then it can be shown that $\left\{y_{r}\right\}_{r}$ accumulates at $y_{\infty}$. Further from the construction of the sets $F_{r}$ it is clear that for any finite $r>0, y_{\infty}$ is at a distance greater than $r$ from $f(X)$. Hence $y_{\infty} \in \cap_{r} F_{r} \subset \partial Y$. Now consider the sets $A:=\overline{f(X)}$ and $B:=\overline{\left\{y_{r}\right\}_{r>1}}$. Clearly $A$ and $B$ are disjoint in $\bar{Y}$. Then by Urysohn's Lemma we construct a Higson function $h \in C(\bar{Y})$, that takes 0 on $A$ and 1 on $B$. Then by continuity $h\left(y_{\infty}\right)=1$ and hence $[h]$ in $C(\partial Y)$ is a non-zero element that is mapped to the zero function in $C(\partial X)$. This is a contradiction that $f$ induces a homeomorphism between the boundaries.

But $f(X)$ is coarsely dense in $Y$ implies that the inclusion map $i: f(X) \rightarrow Y$ of the subspace $f(X) \subset Y$ gives a coarse equivalence. In the other direction we extend the identity map id: $f(X) \subset Y \rightarrow f(X)$ to all of $Y$ by sending $y$ in $\{Y \backslash f(X)\}$ to $f(x)$ for some $x$ such that $(y, f(x))$ belongs to $E$. Then from Corollary 3.29 the boundary of $f(X)$ in $Y$ is homeomorphic to $\partial \bar{Y}$.

Now, for every $y$ in $Y$ such that $y=f(x)$ for some $x$ in $X$, define $g(y):=x$. That is we make a choice. Otherwise, choose $x$ in $X$ such that $(y, f(x))$ belongs to $E$, and define $g(y):=x$. Since $f(X)$ is coarsely dense in $Y$, the map $g: Y \rightarrow X$ is a well-defined map up to the choices made. By its definition, $g$ is bounded, that is, it sends bounded subsets of the coarse structure in $Y$ to bounded subsets of the coarse structure in $X$. We know that $f$ is a coarse map that induces the map $\partial \bar{f}$ at the boundary. By the arguments as in the proof of Lemma 3.25 . all points in the interior of $X$ are mapped by $f$ to the interior in $Y$. Then as $y_{\alpha}$ goes out to the boundary, all its pre-images $f^{-1}\left(y_{\alpha}\right)$ also goes out to the boundary in $\partial \bar{X}$. This shows that $\left.g\right|_{f(X)}$ is proper.

To show $g$ is a coarse map, we first show that $g$ extends to the inverse map $(\partial \bar{f})^{-1}$ between the boundaries. Now consider a point $y_{0}$ on the boundary $\partial \bar{Y}$. Then $Y$ being dense in $\bar{Y}$, there exists a net $\left\{y_{\alpha}\right\}_{\alpha}$ in $Y$ that converges to $y_{0}$. To understand the behaviour of $\left\{g\left(y_{\alpha}\right)\right\}_{\alpha}$, let us first assume that $\left\{y_{\alpha}\right\}_{\alpha} \subset f(X)$. Then for each $\alpha, g\left(y_{\alpha}\right)=x_{\alpha}$ such that $f\left(x_{\alpha}\right)=y_{\alpha}$. For each $\alpha$, we next show that for any choice of $x_{\alpha}$ from the preimage $f^{-1}\left(y_{\alpha}\right),\left\{x_{\alpha}\right\}_{\alpha}$ converges to $(\partial \bar{f})^{-1}\left(y_{0}\right)$. We know that $f$ is a coarse map that induces the map $\partial \bar{f}$ at the boundary. Again by the same arguments as in the proof of Lemma 3.25 . all points in the interior of $X$ are mapped by $f$ to the interior in $Y$. Therefore the accumulation points of $\left\{f^{-1}\left(y_{\alpha}\right)\right\}$ at the boundary $\partial \bar{X}$ shall be in one-toone correspondence with the accumulation points of $\left\{y_{\alpha}\right\}_{\alpha}$ at the boundary $\partial \bar{Y}$ under the homeomorphism $\partial \bar{f}$. But $\left\{y_{\alpha}\right\}_{\alpha}$ converges to $y_{0}$ and therefore $\left\{x_{\alpha}\right\}$ converges to $(\partial \bar{f})^{-1}\left(y_{0}\right)$. Furthermore $E$ being a controlled set of the topological coarse structure on $Y$ from $\bar{Y}$, any net $\left\{y_{\alpha}\right\}_{\alpha} \subset Y$ that has limit points at the boundary can be replaced by a net $\left\{y_{\alpha}^{\prime}\right\}_{\alpha} \subset f(X)$ such that under the map $g$ their image in $X$ have the same limit points in $\partial \bar{X}$. This shows that $g$ is proper since $\left.g\right|_{f(X)}$ is proper. Finally together with the fact that the boundary of $f(X)$ inside $\bar{Y}$ is homeomorphic to $\partial \bar{Y}$ we see that $g$ induces the continuous map $(\partial \bar{f})^{-1}: \partial \bar{Y} \rightarrow \partial \bar{X}$. From Proposition 3.33 we then have that the bounded, proper map $g$ is bornologous.

Once we have that $g: Y \rightarrow X$ is a coarse map, it is now trivial to see that it defines the required inverse to $f$ for it to be a coarse equivalence. First, notice that from the definition of $g$, the set $\{(x, g(f(x))): \forall x \in X\}$ is a controlled set in $(X, \bar{X})$. This is because, if $\left\{x_{\alpha}\right\}_{\alpha}$ converges to a boundary point $x_{0}$ 
in $\partial \bar{X}$ then $\left\{g\left(f\left(x_{\alpha}\right)\right)\right\}_{\alpha}$ should also converge to the same boundary point as $f\left(x_{\alpha}\right)=f\left(g\left(f\left(x_{\alpha}\right)\right)\right)$ for all $\alpha$. Therefore the map $g \circ f$ is close to $i d_{X}: X \rightarrow X$. While the map $f \circ g$ is close to the map $i d_{Y}: Y \rightarrow Y$ by the controlled set $E$. Therefore $f: X \rightarrow Y$ is a coarse equivalence.

Recall that, the coarse structure on $\mathbb{R}^{2 n}$ that we are interested in is the bounded metric coarse structure of its Euclidean metric. By Theorem 1.13 this is the same as the topological coarse structure coming from its Higson compactification. Whereas for the Moyal plane, Rieffel deformation of this Higson compactification defines the noncommutative coarse structure we are interested in. Given that the translation action of $\mathbb{R}^{2 n}$ is trivial on the Higson corona, certain completely positive maps between the Rieffel deformations as defined in 2 induces a *isomorphism between the boundary quotient algebras.

\subsubsection{Noncommutative coarse maps for Rieffel deforma- tion}

In this section we shall study noncommutative coarse maps in the context of noncommutative coarse structures coming from Rieffel deformation of $\mathrm{C}^{*}$-algebras. In the context of 2-cocycle deformation as in [4], we briefly recall the following construction from Chapter 2 Corresponding to an equivariant extension given by a unitization, one can construct an exact sequence of the corresponding crossed product algebras. Then under 2-cocycle deformation, we get an exact sequence of the deformed crossed products. The Landstad algebras of this exact sequence then defines a noncommutative coarse structure on the deformed $\mathrm{C}^{*}$-algebra.

Given a $G$-product as in Definition 2.10 there is a certain construction to extract the Landstad algebra by integrating over the dual action. Taking integrable elements of the undeformed $G$-product and then integrating over the deformed dual action gives a completely positive map from the original $\mathrm{C}^{*}$-algebra to the deformed Landstad algebra. The map is given by,

$$
B \ni b \mapsto \int_{\hat{G}} \hat{\alpha}_{\chi}\left(f_{1} b f_{2}\right) d \chi,
$$

where $(B, \lambda, \hat{\alpha})$ is the original $G$-product and $f_{1}, f_{2} \in C_{0}(\hat{G}) \cap L^{2}(\hat{G})$. For more details one can refer to [4. Remark 2.5].

We expect these completely positive quantization maps between the Rieffel deformations to give examples of noncommutative coarse maps. But the difficulty is that in general they need not restrict to a $*$-homomorphism between the boundary quotient algebras. Fortunately for trivial action on the boundary, we expect to arrive at a $*$-isomorphism as was outlined in Chapter 2. In the next section we see a concrete example of such a noncommutative coarse map.

\subsubsection{An explicit noncommutative coarse map}

Given an anti-symmetric matrix $J$ on $\mathbb{R}^{d}$, one starts with the smooth subalgebra $\mathcal{A} \subset A$ and defines a Moyal-type deformed product ' $\times_{J}$ ' along with a particular 
operator $\mathrm{C}^{*}$-norm to arrive at $\mathcal{A}_{J}$. Completion of $\mathcal{A}_{J}$ with respect to this $\mathrm{C}^{*}$-norm gives the Rieffel deformation $A_{J}$ of $A$. We shall see that there is one particular important completely positive map in this set-up, first defined in [2], very similar in nature to the integration procedure in $[4]$ as was discussed above.

Given a bilinear form, like the metric $g(\cdot, \cdot): \mathbb{R}^{d} \times \mathbb{R}^{d} \rightarrow \mathbb{C}$ on $\mathbb{R}^{d}$, consider the following map $S: \mathcal{A} \rightarrow \mathcal{A}$, defined as

$$
S(a):=\int_{\mathbb{R}^{d}} \alpha_{u}(a) G(u) d u .
$$

For a fixed $\hbar>0$, the function $G(u)$ is the normalized Gauss function on $\mathbb{R}^{d}$,

$$
G(u):=\frac{\sqrt{\operatorname{det} G}}{(\pi \hbar)^{n}} e^{-g(u, u) / \hbar} .
$$

Remark 3.43. By smoothness of the Gaussian function $G(u)$, image of the map $S$ shall be contained inside the smooth subalgebra $\mathcal{A}$ of $A$. By [2, Theorem 4.3], $S: \mathcal{A}_{J} \rightarrow \mathcal{A}$ is continuous with respect to the respective operator $\mathrm{C}^{*}$-norms. Hence we can extend the map to the $\mathrm{C}^{*}$-completions $S: A_{J} \rightarrow A$. This then gives us a quantization map for Rieffel deformation of $A$. Again, considering $A$ as Rieffel deformation of $A_{J}$ corresponding to the anti-symmetric matrix $-J$, we get a similarly defined map $T: A \rightarrow A_{J}$, which coincides with $S$ on the smooth subspace $\mathcal{A}^{11}$

It is shown in 2. Lemma 4.2], that the map $S$ is positive on the smooth subalgebra $\mathcal{A}$.

Lemma 3.44. 2, Lemma 4.2] For $a \in \mathcal{A}$ the following holds

$$
S\left(a^{*} \times_{J} a\right)=\frac{1}{\operatorname{det} G} S\left(a^{*}\right) S(a)+b,
$$

where $b \in \mathcal{A}^{+}$is positive.

But the action $\alpha$ being strongly continuous on $A$, the smooth subalgebra is dense in $A$ with respect to the $\mathrm{C}^{*}$-norm. Hence $S$ being continuous with respect to the $\mathrm{C}^{*}$-norms as was mentioned in Remark 3.43 its extension to the completions shall also be positive. Also the map is completely bounded as discussed in [5. Corollary 2.6] and hence $S: A_{J} \rightarrow A$ is completely positive.

From symmetry, the map in the opposite direction $T$, shall also be completely positive. In our framework our main concern shall be whether this map extends to a map between the unitizations determining the coarse structure of our algebras. For this we would require it to be strictly continuous. Since the map involves convolution with the Gaussian function $G$ with the given action $\alpha$ on the $\mathrm{C}^{*}$-algebra we expect to extend it to the unitization as long as we can extend the action.

Therefore we approach this problem from the other direction. Our examples of noncommutative coarse structures from Rieffel deformation are precisely given

\footnotetext{
${ }^{11}$ As we know from 7 . Theorem 7.1] the smooth subalgebra with respect to the action of $\mathbb{R}^{d}$ on both $A$ and $A_{J}$ respectively are the same.
} 
by an equivariant exact sequence corresponding to some unitization. Therefore we start with the action on the unitization $\bar{A}$, invariant on the essential ideal $A$, so that the map $S: \bar{A}_{J} \rightarrow \bar{A}$ automatically restricts to a strictly continuous completely positive map $S: A_{J} \rightarrow A$. Thus $S: A_{J} \rightarrow A$ so far satisfies the conditions of Definition 3.1 .

To show that $S$ is a coarse map, we shall only need that the given map induces a $*$-homomorphism between the quotient $\mathrm{C}^{*}$-algebras. In general we do not expect this to happen, but for trivial actions we know from Chapter 2 that the Rieffel deformation is the same as the original algebra. And we see that $S$ gives a $*$-isomorphism in this special case.

Lemma 3.45. For trivial action $\alpha$, the map $S: A_{J} \rightarrow A^{12}$ as defined above, is the identity map.

Proof. For the trivial action, $\alpha=$ id of the vector group $V$ on $A$, the whole vector space $A$ is smooth under this action. Hence the integration is defined on the whole of $A$. For $a$ in $A$,

$$
S(a)=\int_{V} G(u) \alpha_{u}(a) d u=\left(\int_{V} G(u) d u\right) a=a .
$$

In particular we claim that for trivial action on the boundary, as in our example of translation action on the Higson corona of the metric coarse structure on $\mathbb{R}^{2 n}$, $S$ defines a noncommutative coarse map.

Lemma 3.46. For noncommutative coarse structure coming from Rieffel deformation with trivial action on the boundary, the map $S: A_{J} \rightarrow A$ gives a *-isomorphism between the boundary quotient algebras.

Proof. After our discussion in the above paragraphs and Lemma 3.45, we now only need to show that the map $S: \bar{A}_{J} \rightarrow \bar{A}$ as defined above restricts to a welldefined map between the boundary quotient algebras with the same formula. For the trivial action, $\alpha=$ id of the vector group $\mathbb{R}^{d}$ on $\bar{A} / A$, the whole vector space $\bar{A} / A$ is smooth under this action. For $a$ in $\overline{\mathcal{A}}$,

$$
S(a)=\int_{V} G(u) \alpha_{u}(a) d u .
$$

We then complete this to the $\mathrm{C}^{*}$-algebra and then define the map on the boundary quotient algebra as the one induced from the unitization by taking its equivalence class. To show the map is well-defined, one needs to show that for any element $a^{\prime}$ in $A$ and $a$ in $\bar{A}$

$$
\left[S\left(a+a^{\prime}\right)\right]=[S(a)]
$$

But this is trivial from the map $S$ being linear and that its restriction to the ideal $A$ is contained in the smooth subalgebra $\mathcal{A}$ of $A$. Then we apply Lemma 3.45 on the boundary quotient algebras, to show that the map $[S]: \bar{A} / A \rightarrow \bar{A} / A$ gives the identity $*$-isomorphism.

\footnotetext{
${ }^{12}$ Note $A_{J}=A$ for $\alpha=$ id
} 
Therefore at least in this situation of trivial action on the boundary, the map $S$ fits in with our definition of a noncommutative coarse map between the noncommutative coarse structures coming from Rieffel deformation. There might be further special conditions with which one can guarantee $[S]$ to be at least a $*$-homomorphism between the boundary quotient algebras and needs to be looked at further. For now, using Lemma 2.28 and Lemma 3.46 we plan to show noncommutative coarse equivalence for the Moyal plane with the classical plane.

\subsubsection{Noncommutative coarse equivalence between the clas- sical plane and the Moyal plane}

Next, we apply the noncommutative coarse maps between Rieffel deformations for trivial action on the boundary to the special case of the metric coarse structure on the classical plane. It is important to note that Lemma 2.29 restricts us to use $S$ as a coarse map only for the noncommutative coarse structure defined by Rieffel deformation of the Higson compactification for the metric coarse structure on $\mathbb{R}^{2 n}$. For other boundaries of $\mathbb{R}^{2 n}$ we need not even have a noncommutative coarse map to the Moyal plane.

On the classical plane, we start with the strongly continuous translation action of $\mathbb{R}^{2 n}$ on the Higson compactification $h \mathbb{R}^{2 n}$ of $\mathbb{R}^{2 n}$. The translation action carries the ideal $C_{0}\left(\mathbb{R}^{2 n}\right)$ to itself, that then by Lemma 2.28 descends to a trivial action on the Higson corona. Further, with trivial action on the boundary, we see above in Lemma 3.46 that the restriction $[S]$ to the boundary quotient algebras shall be the identity map, a $*$-isomorphism. With this we answer our main question for this project.

Theorem 3.47. Consider the noncommutative coarse structure on the Moyal plane, coming from Rieffel deformation of the Higson compactification of the metric coarse structure on $\mathbb{R}^{2 n}$. Then the noncommutative coarse map given by $S: \mathbb{K}\left(L^{2}\left(\mathbb{R}^{2 n}\right)\right) \rightarrow C_{0}\left(\mathbb{R}^{2 n}\right)$ defines a noncommutative coarse equivalence.

Proof. For the inverse noncommutative coarse map we use $T$ as was mentioned in Remark 3.43 . 


\section{Bibliography}

[1] John Roe, Corrections to "Lectures on coarse geometry", August 11, 2005.

[2] Daniel Kaschek, Nikolai Neumaier, and Stefan Waldmann, Complete positivity of Rieffel's deformation quantization by actions of $\mathbb{R}^{d}$, J. Noncommut. Geom. 3 (2009), no. 3, 361-375, DOI 10.4171/JNCG/40 MR2511634 (2011a:53178)

[3] Lawrence G. Brown, Continuity of actions of groups and semigroups on Banach spaces, J. London Math. Soc. (2) 62 (2000), no. 1, 107-116, DOI 10.1112/S0024610700001058 MR1771854 (2003b:22014)

[4] Paweł Kasprzak, Rieffel deformation via crossed products, J. Funct. Anal. 257 (2009), no. 5, 1288-1332, DOI 10.1016/j.jfa.2009.05.013. MR 2541270

[5] Sergey V. Neshveyev, Smooth crossed products of Rieffel's deformations, Lett. Math. Phys. 104 (2014), no. 3, 361-371, DOI 10.1007/s11005-013-0675-9 MR 3164614

[6] Gert K. Pedersen, $C^{*}$-algebras and their automorphism groups, London Mathematical Society Monographs, vol. 14, Academic Press, London, 1979. MR 548006

[7] Marc A. Rieffel, Deformation quantization for actions of $\mathbf{R}^{d}$, Mem. Amer. Math. Soc. 106 (1993), no. 506, x+93, DOI 10.1090/memo/0506 MR 1184061

[8] John Roe, Coarse cohomology and index theory on complete Riemannian manifolds, Mem. Amer. Math. Soc. 104 (1993), no. 497, x+90, DOI 10.1090/memo/0497. MR 1147350

[9] _ Lectures on coarse geometry, University Lecture Series, vol. 31, Amer. Math. Soc., Providence, RI, 2003. MR 2007488 